\title{
SPECTRAL SHAPE DIVISION MULTIPLEXING (SSDM): APPARATUS, TRANSMITTER, RECEIVER AND DETECTION
}

\author{
A Thesis \\ presented to \\ the Faculty of California Polytechnic State University, \\ San Luis Obispo
}

\author{
In Partial Fulfillment \\ of the Requirements for the Degree \\ Master of Science in Electrical Engineering
}

By

Fausto Daniel Holguín-Sánchez

June 2012 
(C) 2012

Fausto Daniel Holguín-Sánchez ALL RIGHTS RESERVED 


\section{Commitee Membership}

TITLE: $\quad$ Spectral Shape Division Multiplexing (SSDM): Apparatus, Transmitter, Receiver and Detection

AUTHOR: $\quad$ Fausto Daniel Holguín-Sánchez

DATE SUBMITTED: Jun, 2012

COMMITTEE CHAIR: John Oliver, Ph. D.

Assistant Professor

COMMITTEE MEMBER: Fred DePiero, Ph. D.

Associate Dean, Assistant Professor

COMMITTEE MEMBER: Dennis Derickson, Ph. D.

Department Chair, Graduate Coordinator 


\title{
Abstract
}

\author{
Spectral Shape Division Multiplexing (SSDM): Apparatus, \\ Transmitter, Receiver and Detection. \\ Fausto Daniel Holguín-Sánchez
}

Wireless communication companies require to use the frequency spectrum to operate. Both frequency licenses and infrastructure to reuse frequencies are costly resources subject to increasing demand. This work introduces a novel multiplexing method that saves spectrum called Spectral Shape Division Multiplexing (SSDM). Under certain configurations, SSDM displays higher flexibility and throughput than other spectrally efficient methods.

SSDM defines the structure of a wireless multi-carrier by software. It is similar to Orthogonal Frequency Division Multiplexing (OFDM) in that both use overlapped subcarriers to make efficient use of allocated spectrum. However, SSDM has several advantages. Where OFDM organizes sub-carriers orthogonally, SSDM allows arbitrary frequency steps enabling higher spectral efficiency. Similarly, while OFDM and other spectrally efficient methods use sinusoidal pulse forms, SSDM can use non-standard pulses providing a greater control of the carrier. In this thesis, a SSDM transceiver is implemented to reduce the spectrum utilization. SSDM presents an increase in spectral efficiency of $20 \%$ average with respect to OFDM. The cost of this gain is higher computational speed and signal to noise ratio. 
The mathematical models and possible architecture for an SSDM system with sinusoidal pulses is developed. The modem is compared with other spectrally efficient methods. Similarly, the trade-offs between spectral efficiency, bit-error rates, dimension of the carrier and sub-carrier spacing are subject of analysis.

Keywords: shared spectrum multiplexing SSM, pulse shape division multiplexing PSDM, non orthogonal frequency division multiplexing NOFDM, spectrally efficient frequency division multiplexing SEFDM, overlapped frequency division multiplexing OvFDM, signal superposition transceiver. 


\section{Acknowledgements}

To Patricia, Antonio, Ricardo, Carlos Andrés, Mónica, María José, Cristina, Olga Vásconez and all my beloved family.

In memory of María Herminia Giraud, Miguel Sánchez, and Fausto Holguín.

To Sarah Devlin, Miki Vasconez and Elenita Laurich, my Newman friends and all the others in San Luis Obispo who took care of me during my studies.

Thanks to Drs. John Oliver, Fred DePiero, Dennis Derickson, Jerome Breintenbach and Wayne Pilkington for the qualitative time spent towards the fulfillment of my degree.

\section{Agradecimientos}

Con todo cariño para Patty, Toño, Ricky, Carlos Andrés, Móni, a mi ahijada María José, a Titi y a mi abuelita Olga que me han dado todo.

En memoria de mis abuelos Maruja Giraud, Miguel Sánchez y Fausto “El Pastas" Holguín.

Dedicado a Sarah Devlin, a Miki Vásconez y Elenita Laurich, a mis amigos en Newman y en San Luis Obispo que me han cuidado durante mis estudios.

Gracias a mis profesores John Oliver, Vladimir Prodanov, Dennis Derickson, Fred DePiero, Wayne Pilkington y Jerome Breintenbach por su continuo soporte. 


\section{Contents}

List of Tables $\quad x$

List of Figures $\quad$ xii

1 Introduction 1

1.1 The concept of SSDM . . . . . . . . . . . . . . . 1

1.2 Thesis organization . . . . . . . . . . . . . . 2

1.3 List of acronyms . . . . . . . . . . . . . . . 3

2 Basics of communications 5

2.1 Communication codes . . . . . . . . . . . . . . 5

2.1.1 The use of modulation codes _. . . . . . . . . . . . 5

2.1.2 Quadrature Amplitude Modulation (QAM) . . . . . . . . . 6

2.1 .3 SSDM as a code ................... 9

2.2 Challenges in wireless communications . . . . . . . . . . . . . 12

2.2.1 Multipath propagation . . . . . . . . . . . . 13

2.2.2 Time-frequency selective channels . . . . . . . . . . . . . . . 14

2.2.3 Doppler effect . . . . . . . . . . . . . . . 15

2.2.4 Summary of wireless communications challenges . . . . . . . . 16

3 Related work $\quad 18$

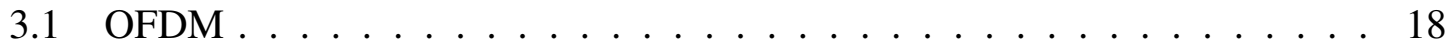

3.2 Spectrally efficient $(\mathrm{SE})$ methods . . . . . . . . . . . . . . 25

3.2 .1 OvFDM, SEFDM . . . . . . . . . . . . . 25 
3.2.1.1 Maximum likelihood (ML) detector . . . . . . . . . . . 28

3.2.1.2 Sphere decoders (SD) [23] . . . . . . . . . . . . . . . 29

3.2.1.3 Fixed Sphere Decoder (FSD) [20] . . . . . . . . . . . 29

3.2.1.4 Interleaved overlapped SEFDM with stripe detector [8] . 29

3.2.1.5 Truncated Singular Value Decomposition (TSVD) detector $[21,18] \ldots \ldots \ldots \ldots$

3.2.1.6 Fast Constrained Sphere Decoder (FSD) [26] . . . . . . 32

3.2.1.7 Precoded SEFDM detector [19] . . . . . . . . . . . . 32

3.2 .2 Non OFDM (NOFDM) . . . . . . . . . . . . . . . . . . . 32

3.2 .3 Other systems . . . . . . . . . . . . . . . . 33

4 SSDM 35

4.1 Scope . . . . . . . . . . . . . . . . . . 35

4.2 Theoretical analysis . . . . . . . . . . . . . . . 36

4.2.1 Sub-carriers with uniform standard pulses . . . . . . . . . 37

4.2.1.1 Discrete analysis . . . . . . . . . . . . . . 42

4.2.1.2 Matrix example for formula derivation . . . . . . . . 43

4.2.2 Sub-carriers with non standard pulse shapes . . . . . . . . . . 48

4.2.2.1 Hanning RF pulse . . . . . . . . . . . . . . . . 48

4.2.2.2 Heterogeneous sub-carriers . . . . . . . . . . . 50

5 Implementation $\quad 52$

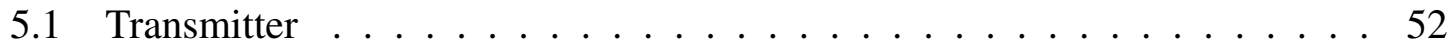

5.2 Receiver . . . . . . . . . . . . . . . . . . 54

6 Experimental Model Set-Up $\quad 58$

6.1 Monte-Carlo analysis . . . . . . . . . . . . . . . . 58

6.2 Algorithm . . . . . . . . . . . . . . . . . . . . 59

6.3 Tests . . . . . . . . . . . . . . . . 60

7 Results $\quad 62$

7.1 Comparison with OFDM $\ldots \ldots \ldots \ldots \ldots$ 
7.2 Performance . . . . . . . . . . . . . . . . . 62

7.3 Comparison with other methods . . . . . . . . . . 65

7.4 Future work . . . . . . . . . . . . . . 70

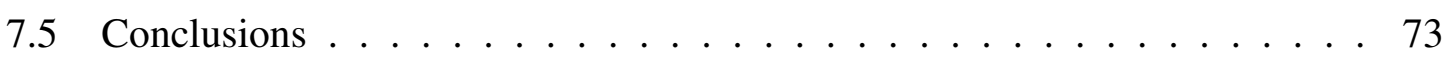

$\begin{array}{ll}\text { Bibliography } & 77\end{array}$ 


\section{List of Tables}

3.1802 .11 a system parameters $\ldots \ldots \ldots \ldots \ldots . \ldots \ldots 22$

3.2 Overlapped frequency detectors comparison . . . . . . . . . . . 27

4.1 Components of an SSDM subcarrier spectra $\ldots \ldots \ldots \ldots . \ldots . \ldots 42$

4.2 SSDM modulation in the time domain . . . . . . . . . . . . 43

4.3 SSDM modulation in the frequency domain $\ldots \ldots \ldots \ldots$

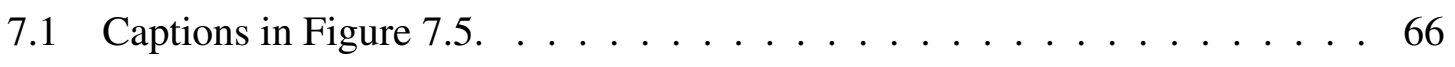

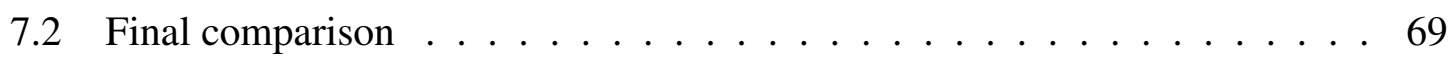




\section{List of Figures}

2.1 Morse code . . . . . . . . . . . . . . . . . . . . 6

2.2 Symbols in $16-\mathrm{QAM} \ldots \ldots \ldots \ldots \ldots \ldots \ldots$

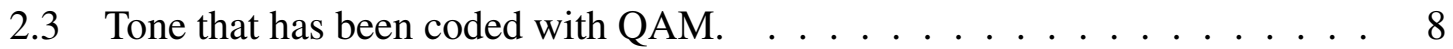

2.4 Arbitrary QAM constellations maps $M_{i}$ and their order $\ldots \ldots \ldots$

2.5 The SSDM code . . . . . . . . . . . . . . . . . . . . . . 9

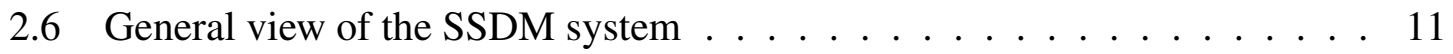

2.7 Multipath in wireless communications . . . . . . . . . . . . . 13

2.8 ISI due to multipath propagation $\ldots \ldots \ldots \ldots \ldots$

2.9 Response of time and frequency selective channels $\ldots \ldots \ldots \ldots$

2.10 Doppler effect . . . . . . . . . . . . . . . . 16

3.1 Orthogonal Frequency Division Multiplexing (OFDM) $\ldots \ldots \ldots \ldots$

3.2 Power spectrum of 16 OFDM subcarriers. . . . . . . . . . . . 20

3.3 High peak to average ratio due to subcarriers overlapping in SE methods . . 20

3.4 ICI in OFDM due to Doppler effect . . . . . . . . . . . . . . . 21

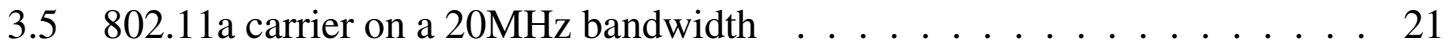

3.6 OFDM transmitter $\ldots \ldots \ldots \ldots \ldots \ldots$

3.7 OFDM receiver $\ldots \ldots \ldots \ldots \ldots \ldots$

3.8 Timing in OFDM . . . . . . . . . . . . . . . . . . . . . . 24

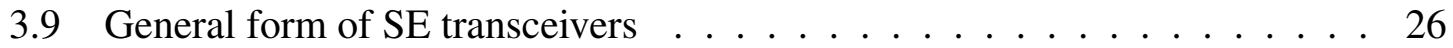

3.10 Spectrum bandwidth use, OFDM vs SE methods . . . . . . . . . . 28

3.11 Interleaved overlapped SEFDM transmitter . . . . . . . . . . . 30

3.12 NOFDM carrier . . . . . . . . . . . . . . . . . . . . . . 33

$3.13 \mathrm{SEFDM} / \mathrm{OvFDM} / \mathrm{SSDM}$ carrier $\ldots \ldots \ldots \ldots \ldots . \ldots . \ldots . \ldots$ 
4.1 SSDM and other SE systems . . . . . . . . . . . . . . . 36

4.2 Normalized components of the spectral shape of a subcarrier . . . . . . . . 39

4.3 Complex spectral shape of a subcarrier and its components . . . . . . . . . 40

4.4 Relationship between $f_{i}$ and $1 / T \ldots \ldots \ldots \ldots$. . . . . . . . . 41

4.5 Homogeneous and heterogeneous subcarriers . . . . . . . . . . 51

5.1 SSDM transmitter example . . . . . . . . . . . . . . . . . . 53

5.2 SSDM receiver . . . . . . . . . . . . . . . . . . 57

6.1 Monte-Carlo analysis on the SSDM system model . . . . . . . . . . . . . 59

$7.1 \quad$ BER vs $\mathrm{E}_{b} / \mathrm{N}_{0} \mathrm{vs} \alpha \ldots \ldots \ldots \ldots \ldots$

7.2 Effective SE and percentile throughput gain with respect to OFDM . . . . . 65

7.3 BER tendency by carrier dimension . . . . . . . . . . . . 66

7.4 Percentile throughput gain of SSDM over OFDM vs carrier dimension . . . 67

7.5 Performance comparison with other receivers with Q-PSK . . . . . . . 68

7.6 SSDM error rate BER in relation to the sampling frequency $f_{S} \ldots \ldots 9$

7.7 Slope change in regular and a widowed SSDM signal . . . . . . . . . . 71

7.8 Application of a Hanning window on a symbol . . . . . . . . . . 71

7.9 Concentration of the spectral energy before and after windowing . . . . . 72 


\section{Chapter 1}

\section{Introduction}

Frequency bands are an expensive resource subject to a greater demand everyday $[14,13$, 32]. Due to this fact, the capacity of wireless networks becomes rapidly exhausted in congested areas $[12,11,10]$. To maintain the service quality in zones that show high demand, telecommunication operator companies (Telcos) take actions to keep the tradeoff between capacity and coverage [32]. One of these actions is upgrading the wireless infrastructure to recycle assigned frequencies in smaller coverage areas. Another action is to buy more licenses to use additional radio-electric spectrum. Both of these alternatives are expensive for Telcos. For instance, approximately $\$ 19$ billion were invested in 2008 for the $700 \mathrm{MHz}$ band in the United States only [16]. Similarly, "the global wireless industry spends $\$ 50$ billion to upgrade" their wireless networks every year [9]. SSDM helps both to save costs in pricy spectrum and to mitigate the need of deploying additional infrastructure when the frequencies are scarce.

\subsection{The concept of SSDM}

Spectral shape division multiplexing (SSDM) is a modulation technique that can both optimize the utilization of the scarce radioelectric spectrum and increase the data throughput. Similar to OFDM, multiple sub-carriers can be used to evenly distribute information across an allocated bandwidth. However, SSDM also allows these sub-carriers to be nonorthogonal, allowing more carriers per designated bandwidth. The main tradeoff of using 
non-orthogonal carriers is a higher bit-error rate for a fixed signal-to-noise ratio. Despite higher bit-error rates, it is shown that SSDM out-performs OFDM and other modulation techniques in effective spectral efficiency (ESE), which is defined as the number of successful bits per second per Hertz that can be achieved.

SSDM is a transceiver able of encode and decode, multiplex and demultiplex, digital multicarrier signals with any pulse shape, any modulation frequency and any constellation order in each subcarrier - as long as every subcarrier has a unique pulse shape. Therefore, the system has been named Spectral Shape Division Multiplexing meaning that it multiplexes streams of information by assigning unique spectral forms to every stream. Other names that could work for the same purpose are Linear Independent Frequency Division Multiplexing (LIFDM), Shared Spectrum Multiplexing (SSM) or Pulse Shape Division Multiplexing (PSDM).

\subsection{Thesis organization}

Chapter 2 talks about the basics of modulation and codification techniques, the challenges of communications and provides an introduction to the SSDM signaling. Chapter 3 discusses OFDM and other modulation techniques geared towards spectral efficiency. The theoretical background of an SSDM transceiver is described in Chapter 4. Chapter 5 depicts a possible design of an SSDM transceiver. Chapter 6 explains the model used to evaluate the performance of the SSDM system. Finally, Chapter 7 compares the results of the SSDM transceivers with other spectrally efficient methods. 


\subsection{List of acronyms}

4G Fourth generation of mobile networks

ADC Analog to digital converter

AMC Adaptive modulation and coding

AWGN Additive white Gaussian noise

BER Bit error rate

BW Bandwidth

CP Cyclic prefix, cyclic postfix

DAC Digital to analog converter

DSP Digital signal processing

DVB-T Digital video broadcasting - terrestrial

ESE Effective spectral efficiency

FDM Frequency division multiplexing

FFT Fast Fourier transform

FrDFT Fractional discrete Fourier transform

FSD Fixed complexity sphere decoder

GS Gram Schmidt orthonormalisation procedure

ICI Inter-carrier interference

IFFT Inverse fast Fourier transform

IFrDFT Inverse fractional discrete Fourier transform

IMT International Mobile Telecommunications

ISI Inter-symbol interference

LTE Long term evolution

MAC Medium access control 


\begin{tabular}{ll} 
MIMO & Multiple input multiple output \\
ML & Maximum likelihood \\
MMSE & Minimum mean square error \\
NOFDM & Non orthogonal frequency division multiplexing \\
OFDM & Orthogonal frequency division multiplexing \\
OvFDM & Overlapped frequency division multiplexing \\
PC & Personal computer \\
PCM & Pulse code modulation \\
P/S & Parallel to serial \\
QAM & Quadrature amplitude modulation \\
QPSK & Quadrature Phase Shift Keying \\
RF & Radio-frequency \\
RSD & Real-valued sphere decoder \\
SD & Sphere decoder \\
SE & Spectral efficiency, spectrally efficient \\
SNR & Signal to noise ratio \\
SSDM & Spectral shape division multiplexing \\
SSM & Spectrum sharing multiplexing, SSDM \\
SEFDM & Spectral efficient frequency division multiplexing \\
SIMO & Single input multiple output \\
S/P & Serial to parallel \\
TDM & Time division multiplexing \\
TSVD & Truncated singular value decomposition \\
WiMAX & Worldwide interoperability for microwave access \\
WLAN & Wireless local area network \\
ZF & Zero forcing \\
\hline
\end{tabular}




\section{Chapter 2}

\section{Basics of communications}

\subsection{Communication codes}

\subsubsection{The use of modulation codes}

According to the Cambridge Dictionary a code is "a system for conveying information with signs or symbols that are not" necessarily "ordinary language or the signs or symbols themselves". Accordingly, modulation means varying "the strength, tone, or pitch ... of an electromagnetic wave or other oscillation in accordance with the variations of a second signal, typically one of a lower frequency". The combination of these two is broadly used in communications to send messages from a transmitter to a receiver. Figure 2.1 represents an example of a code used for communications. Finally, a symbol is defined as "a mark or character used as a conventional representation of an object, function, or process". In communications, a symbol is a piece of information that has been codified.

Wireless systems use communication codes broadly. Morse code, for example, is a modulating code that can be used with either light or sound waves. Morse modulates the amplitude 1 of the signal ON and OFF during the transmission of the message. Similarly, to construe messages properly, wireless transceivers share a common modulation code. This code can be standard so anyone can build radios to modulate in that particular code. Codes can also be proprietary for security purposes.

\footnotetext{
${ }^{1}$ Signal strength or signal power
} 


\begin{tabular}{|llllll|}
\hline A & $\cdot-$ & J & $\cdot---$ & S & $\cdots$ \\
B & $-\cdots$ & K & $-\cdot-$ & T & - \\
C & $-\cdot-\cdot$ & L & $\cdot-\cdot$ & U & $\cdots-$ \\
D & $-\cdot$ & M & -- & V & $\cdots-$ \\
E & $\cdot$ & N & $-\cdot$ & W & $\cdot--$ \\
F & $\cdots-\cdot$ & O & --- & X & $-\cdot-$ \\
G & $--\cdot$ & P & $\cdot--\cdot$ & Y & $-\cdot--$ \\
H & $\cdots$ & Q & $--\cdot-$ & Z & $--\cdot$ \\
I & $\cdots$ & R & $\cdot-\cdot$ & & \\
\hline
\end{tabular}

Figure 2.1: Morse code

\subsubsection{Quadrature Amplitude Modulation (QAM)}

In the same way than Morse uses a table to convert an alphabetic symbol to codified information, QAM requires the definition of mapping tables. These tables are called constellation maps and need to be the same in all the transceivers. In contrast with Morse code, the constellations in QAM can have more than two possible states (ON/OFF). In this sense, the signal is modulated using various amplitude levels, as defined by constellation. On top of that, QAM modifies the phase of the signal independently of the amplitude. The possible combinations of amplitude-phase determine the number of symbols that can be represented in a codified signal.

Figure 2.2 shows an example of a QAM constellation. In this example, groups of 4 bits of information have been assigned to every constellation element. These elements are points on a complex plane with arbitrary arrangement. In the practice, many different forms could be adopted according to the convenience of the case. The number of elements in the constellation defines its order which is $2^{M}$; where $M$ is the amount of bits that are grouped together at a time to form a symbol. Since in this example $2^{M}=16$, this type of modulation is called 16-QAM.

A complex vector in Figure 2.2 represents the constellation symbol to be transmitted; from which, the magnitude $A$ represents the amplitude and $\phi$ the phase of the modulated 


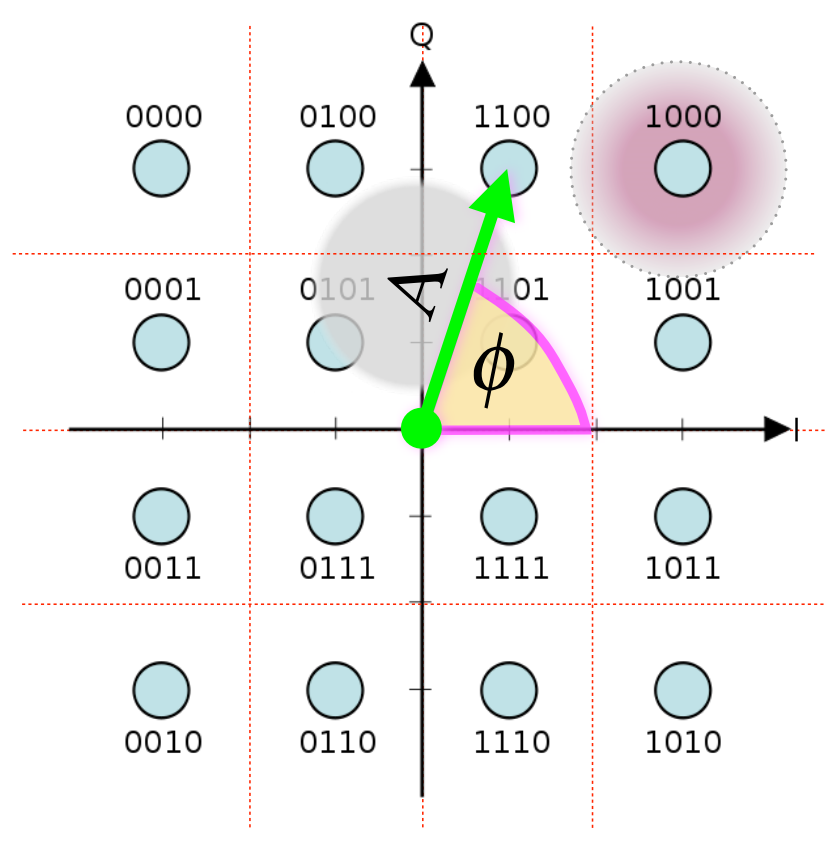

Figure 2.2: Symbols in 16-QAM

The amplitude $A$ and the phase $\phi$ of a signal are modified according to the constellation.

signal. Historically, the axis $I$ and $Q$ represent In-phase and Quadrature-phase respectively ${ }^{2}$. The mathematical representation of a normalized carrier signal $r_{0}(t)=\sin \left(2 \pi f_{0} t\right)$ that has been modulated with QAM is $s(t)=A \sin \left(2 \pi f_{0} t+\phi\right)$. This expression has an equivalent rectangular representation $s(t)=A_{R} \sin \left(2 \pi f_{0} t\right)+A_{I} \cos \left(2 \pi f_{0} t\right)$ where $A_{R}$ and $A_{I}$ are the real and imaginary components ${ }^{3}$. In other terms, $A_{R}=A \cos (\phi)$ and $A_{I}=A \sin (\phi), A=\sqrt{A_{R}^{2}+A_{I}^{2}}$ and $\phi=\tan ^{-1}\left(A_{I} / A_{R}\right)$.

To demodulate the signal, the receiver uses the exact same constellation than the transmitter. However, during its way to the receiver, the signal is affected by several types of corruption (described later in Section 2.2 on page 12). Because of this, the vector coordinates of the received symbol may be different than the ones from the original symbol. In fact, the received symbols tend to display a two-dimensional Gaussian distribution in the complex plane as shown in the shaded area by the element 1000 in Figure $2.2^{4}$. The

\footnotetext{
${ }^{2}$ For the rest of this work, the subindex $I$ will refer to the imaginary part. Meanwhile, $Q_{i}$ represents the complex vector $A e^{j \phi}$ and is also used indistinctly to recall a constellation map.

3 ... projections of $A$ into the axis.

${ }^{4}$ The distribution of the received symbols is not always circular. It depends on several factors such as brown noise, phase noise or linearity of the amplifiers.
} 
segmented lines in red delineate what are called the decision regions. A symbol that is received within a decision region is associated to its corresponding element. Errors are produced when a symbol is deflected beyond the boundaries of a decision region.

An example of a signal that has been modulated with QAM is depicted in Figure 2.3. Groups of bits have been matched in a constellation map to vary both the phase and the amplitude of the carrier signal which in this case is a sine tone with a specific frequency. For purposes of this example, the symbol time is $T=0.5$ (arbitrary units). The bit rate of this carrier is then $M / T$ bps. However, this signal seems to have discontinuities, in real life it is a continuous function. The transitions marked in red at the end of every $T$ are smoothed by several factors like for instance the transition time of the switching elements in the amplifier or parasitic capacitances. That effect causes Inter-Symbol Interference (ISI) that is further explained in Section 2.2.1 on page 13.

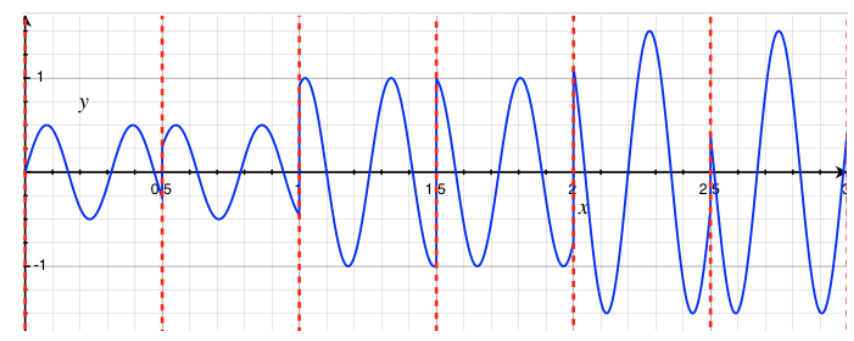

Figure 2.3: Tone that has been coded with QAM.

The constellation maps in QAM are designed based on technical or security purposes. Figure 2.4 shows some of these possibilities. Scattered and circular symmetrical forms are the most used in the practice. $M_{2}$ and $M_{4}$ for instance are denominated circular 8-QAM and rectangular rotated 8-QAM respectively. Similarly, $M_{1}$ and $M_{3}$ are both 16-QAM so $M_{5}$ and $M_{8}$ are both 4-QAM.
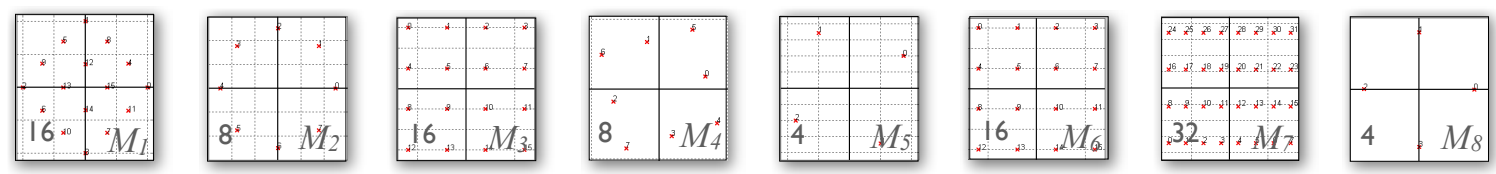

Figure 2.4: Arbitrary QAM constellations maps $M_{i}$ and their order 


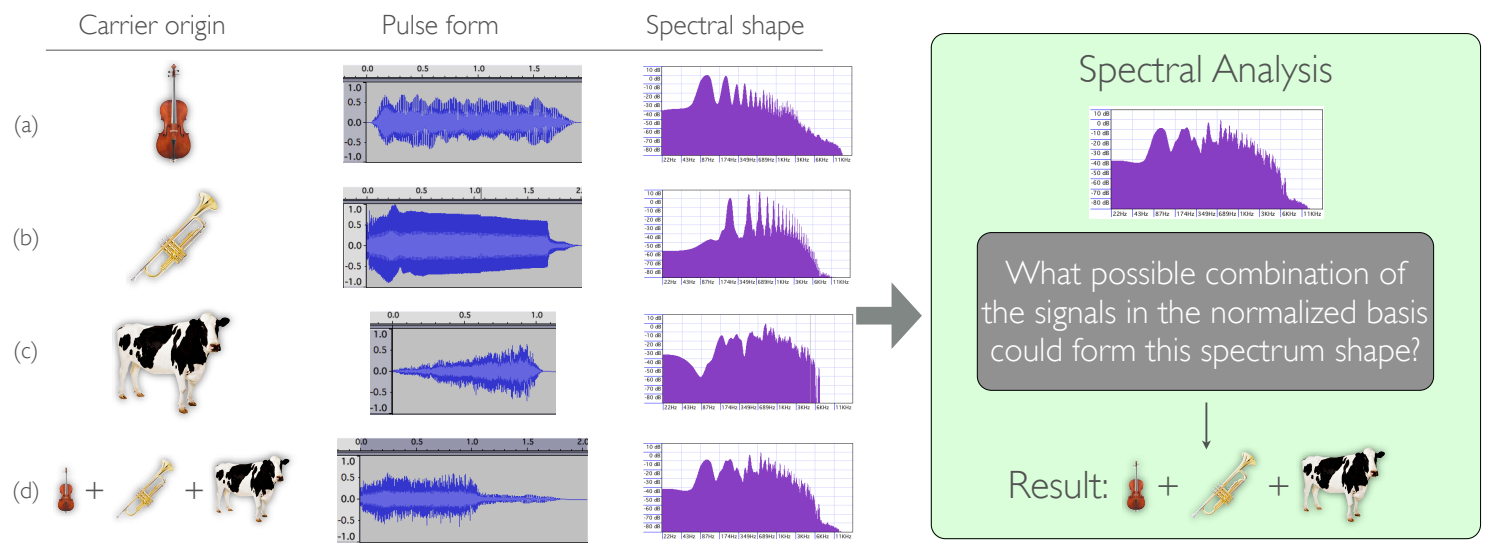

Figure 2.5: The SSDM code

\subsubsection{SSDM as a code}

To introduce the fundamental idea of SSDM signaling, it is convenient to consider how humans are able to differentiate simultaneous sounds. As an example, the column titled Pulse Form in Figure 2.5 shows several waveforms and their combination - in this case, the sound waves of a cello, a trumpet and the bellowing of a cow. The signal in (d) corresponds to the linear combination of those signals in different proportions. In this example, the signal of the cello, so its icon, has been scaled by a factor of 0.5 . The resultant signal in (d) corresponds to only one possible combination of the former signals. This is $0.5(a)+$ $(b)+(c)$. Could (a) itself make the combined sound in (d)? No, the unique wave shapes in (d) can be obtained only by a particular combination of (a), (b) and (c). If those signals are analyzed in the frequency domain, it can be said that they have all have a different spectral shapes. Therefore, a radio should be equally able to differentiate signal waves, despite interfering, because they all have different wave patterns. The reverse process to do so is shown in the right part of Figure 2.5.

The characteristic patterns of a wave can be seen in both the time and frequency domains due to the duality property of the Fourier transform that makes the domains virtually symmetrical [36]. Based on the idea of characteristic pattern superposition, the SSDM modem is able to differentiate and properly decode blended signals. Additionally, the SSDM receiver focuses its analysis in the frequency domain because it displays greater shielding to noise and easier signal manipulation. 
By definition, a multiplexer is a system that sends several messages at once. The multiplexing technique that characterizes SSDM is that multiple messages are sent through the same channel with different spectral shapes (or pulse shapes). The combination of the multiple signals produce a composite that is unique. The shape of the waves used is not limited in time or frequency as long as they are linearly independent and fit in the symbol period $T$. For the SSDM transceiver, the selected waveforms form part of a normalized basis ${ }^{5}$ that is used to encode and decode the messages. The normalized basis is like a toolbox that both the transmitter and the receiver use to construct and model the carrier signal respectively. The basis can be stored in the transceiver in either domain, time or frequency.

The SSDM system comprises of a transmitter and a receiver as shown in Figure 2.6. The transmitter in the upper left side of Figure 2.6 shows an example of how groups of 4 bits have been grabbed from an information stream. Those groups are used to map the symbols in the QAM constellation maps. Different spectral forms are obtained by using QAM on the subcarrier base pulse-forms stored at the transmitter. The spectrum of the transmitted signal responds to the overall superposition of the previous forms. The basis waveforms for every subcarrier have been stored in the frequency domain representation. The actual constellations would have $M=2^{k}=16$ elements where $k$ is the number of bits grouped, however only 4 of them are represented in the figure.

At the receiver, the spectrum might have been corrupted with noise, distortion and attenuation. The receiver projects this received spectrum on a normalized basis. This basis has been represented with a circle (receiver in Figure 2.6) comprising all the normalized spectral forms of the subcarriers. The shadow of the incoming signal on the circle represents the projection which is in general a mathematical operation. The result of the projection is an approximation of the original symbols. A classifier, not shown in the figure, regenerates and organizes the information bits.

Continuing with Figure 2.6, the transmitter has $N=3$ subcarriers of order of $M=$ 16 each. In other words, groups of 12 bits of information are taken and divided in $N$ groups of $k_{i}$ bits each. In this case $k_{1}=k_{2}=k_{3}=4$. These bit groups are mapped on constellation maps with 16 elements each to define complex constants $Q_{i}$ that modulate

\footnotetext{
${ }^{5}$ Not limited to orthonormal bases that are broadly used in mathematics.
} 


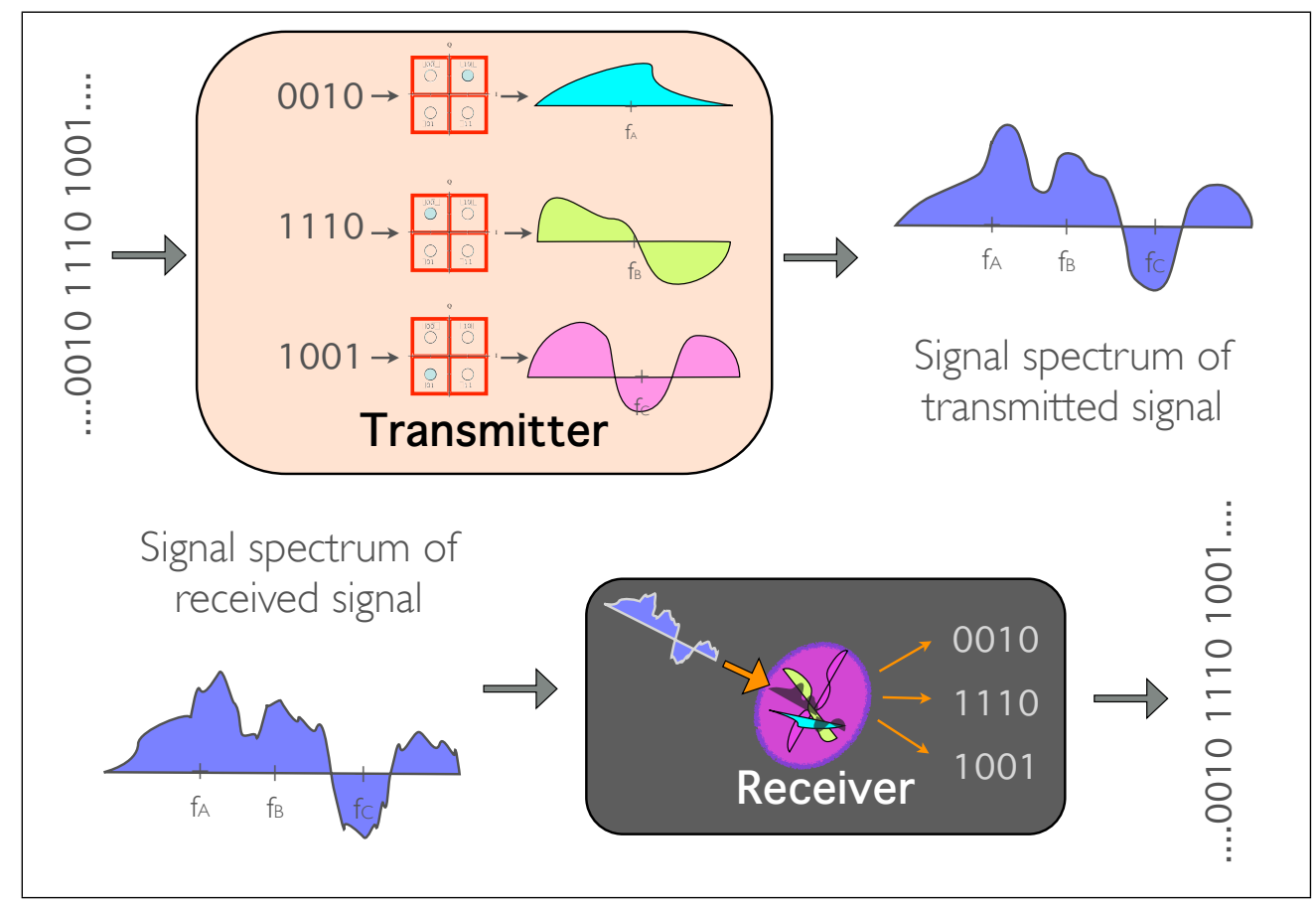

Figure 2.6: General view of the SSDM system

the subcarriers base pulses with QAM. The sum of these subcarriers pulses composes the transmitted signal which spectrum is shown in the figure.

In this system, the transmitter takes a slice of information of $\sum_{i=1}^{N} k_{i}$ bits that is divided in $N$ groups of $k_{i}$ bits each, where $N$ is the number of subcarriers, $i$ is the number of a specific subcarrier and $M_{i}=2^{k_{i}}$ is the order of the constellation of the subcarrier $i$. The grouped bits for each carrier are mapped on the constellation $i$ to modulate a normalized subcarrier signal $r_{i}(t)$ generating $s_{i}(t)$. The signal sent by the transmitter is $s(t)=\sum_{i=1}^{N} s_{i}(t)$.

On the other end, the receiver takes the corrupted spectrum of the received signal $\mathscr{F}\left\{s^{\prime}(t)\right\}$ where $\mathscr{F}\{\ldots\}$ represents the Fourier transform and $s^{\prime}(t)$ the received signal. The receiver projects this spectrum onto the normalized basis which gives in return the modulation constants $Q_{i}^{\prime}$. These constants are mapped back at the original constellations by a classifier that decides what symbol approximates better to the received modulation constants. The receiver puts the received symbols back together, completing the decoding loop. 
This completes the concept of signaling with the SSDM code. The SSDM communications system is described in detail in Chapter 4 on page 35 .

\subsection{Challenges in wireless communications}

There are several difficulties in free space communications that need be considered when designing a system. Some of these are [29, 15]:

- Envelope fading

- White noise, phase noise, brown noise

- Interference

- Multi-path propagation

- Time dispersive channel

- Frequency selective channel, and

- Doppler effect

Apart from those, there are also problems at the radio amplifier:

- Linearity

- Peak to Average Power Ratio (PAPR)

Generally speaking, the methods used to deal with the previous difficulties are $[29,15]$ :

- Digital calibration

- Cyclic redundancy

- Frequency synchronization

- Channel estimation

- Codification

- Forward error correction

- Time synchronization

- I/Q imbalance compensation, and

- Phase noise compensation

The following subsections give a summary of the most important effects to consider when designing a system for wireless communications. 


\subsubsection{Multipath propagation}

In wireless environments, every time a wireless wave hits an object the signal is reflected ${ }^{6}$. Figure 2.7 simplifies this phenomenon which is named multipath propagation [30]. Because of multipath propagation, the signals received from the free space can be seen as the superposition of an undefined number of attenuated copies. Those copies arrive at different times and have taken indistinct trajectories. The resultant signal is a spread version of the original.

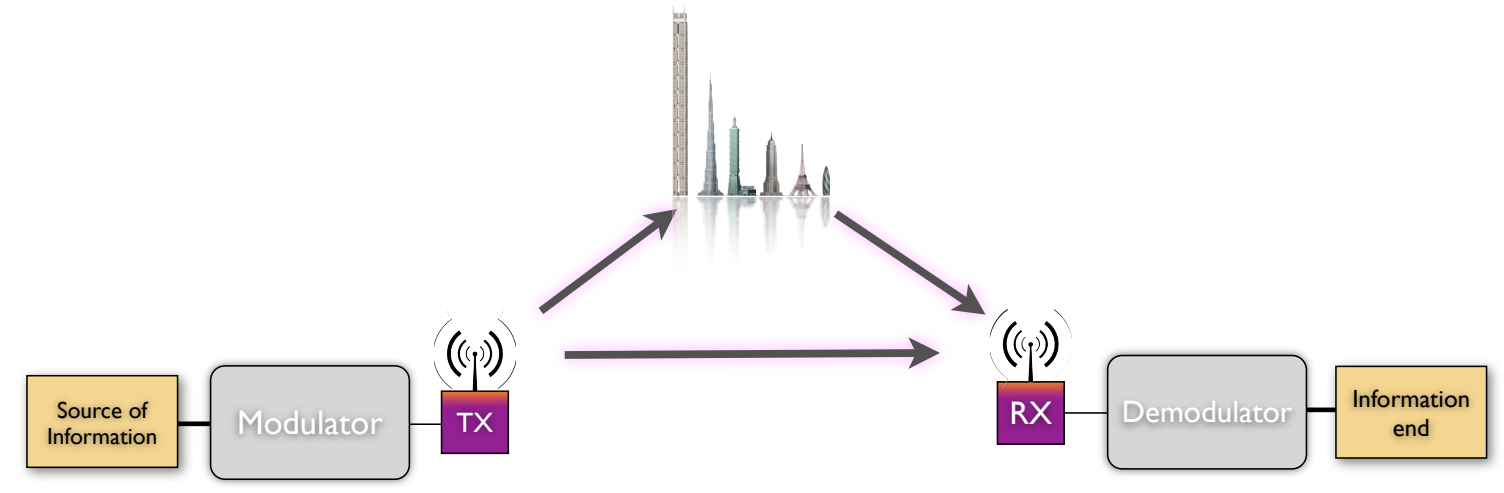

Figure 2.7: Multipath in wireless communications

The time that takes from the moment the transmitter first inserts the signal into the medium to the moment when all the copies have arrived is the maximum time delay $\tau_{\max }$ of the channel. Figure 2.8 shows four copies of a signal that arrive to the receiver through different paths. At the receiver, the signals have different amplitude and time shift. This effect causes an unpredictable response within the time delay of the channel due to ISI. Nevertheless, after $\tau_{\max }$, the strength and integrity of the resultant signal is stable assuming that no other variations exist in the channel. In general, high speed carriers are more sensitive to ISI.

To tackle the effect of multi-path propagation, wireless systems have a signal period longer than $\tau_{\max }$. To do this, transmitters today make the symbols longer by inserting information either at the beginning or at the end of the period. This extra information is called

\footnotetext{
${ }^{6}$ along with other effects classified as transmission, scattering and diffraction [30]
} 


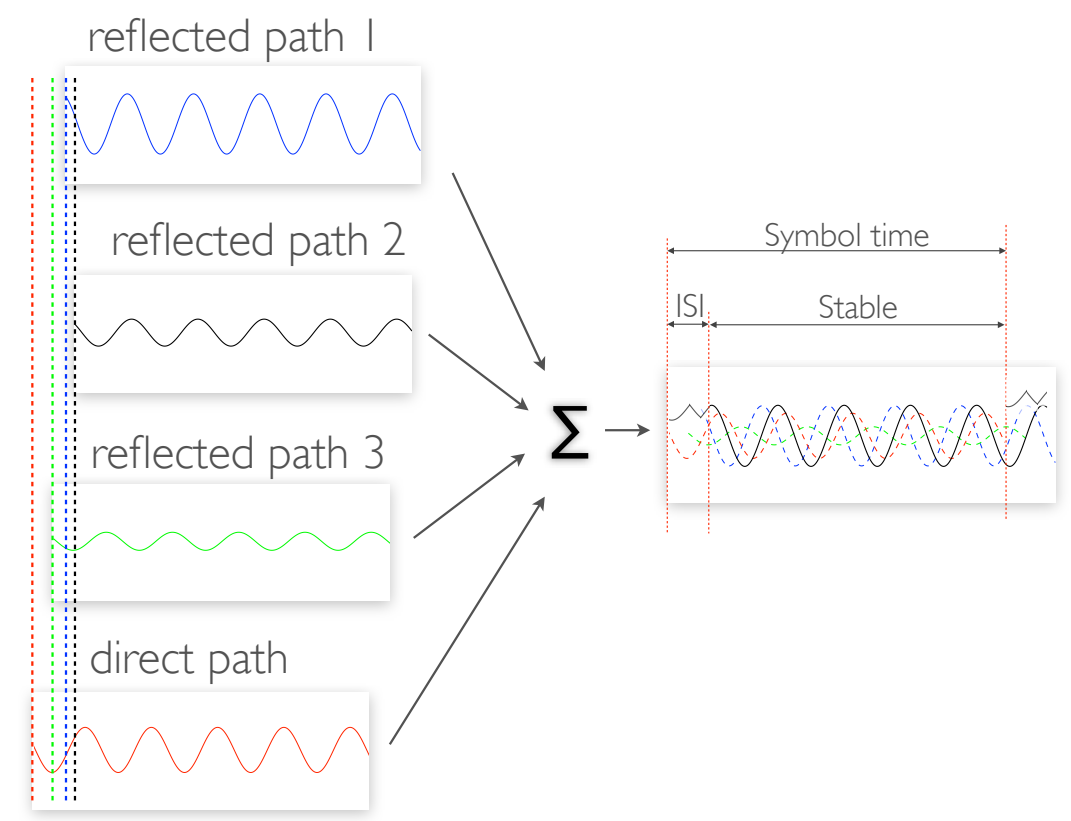

Figure 2.8: ISI due to multipath propagation

cyclic prefix $(\mathrm{CP})$ or cyclic postfix respectively. Accordingly, receivers track the beginning of the signal, discard the part that displays ISI and keep the stable part for demodulation.

\subsubsection{Time-frequency selective channels}

Either time selective or frequency selective, distorting channels have an effect on the signals in both time and frequency domains. A time selective channel is the one that has an attenuation response that changes considerably along the time. Similarly, a frequency selective channel is the one that has a different attenuation levels at different frequencies. 

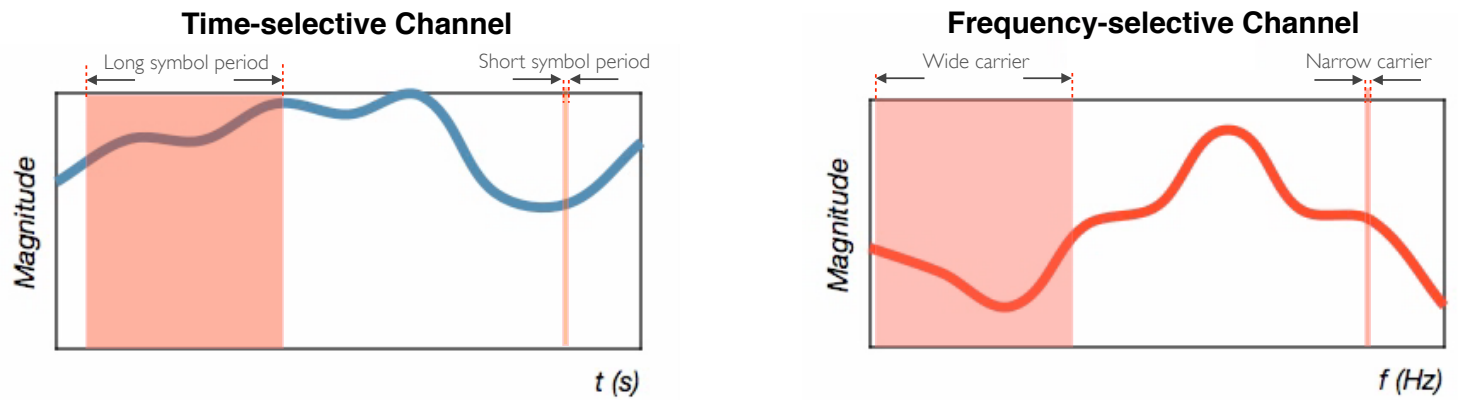

Figure 2.9: Response of time and frequency selective channels

Figure 2.9 shows an example of the behavior of time and frequency selective channels. In the time selective channel, a symbol with a short period would experience less distortion than a symbol with a long period. Therefore, the symbols are usually designed to be short. By doing that, the attenuation of the signal is considered constant through all the signal period.

On the other hand, in the frequency selective channel shown in Figure 2.9, a narrow carrier would experience less distortion than a wide carrier. A mechanism to avoid the distortion effects of a frequency selective channel is to use narrow band channels. Conversely, a wide band channel would require equalization at the receiver whereas the attenuation of a narrowband channel can be considered constant through all its spectrum.

\subsubsection{Doppler effect}

Wireless communications opens the door to mobile communications. A challenge that is derived from mobility is the Doppler effect. The Doppler effect exists when one terminal is moving with respect to the other. This produces either the compression or the elongation of the waves depending on whether the terminals are approaching or moving away. Figure 2.10 shows a static and a moving transmitter. In both of the graphs, the outermost wave was generated when the transmitter was at coordinates $(0,0)$. In $(a)$, the frequency of the signals for a potential receiver at any coordinates is always the same. However, in (b), the frequency of the received signals will be shifted depending on the location of the receiver. The Doppler shift is calculated by $D_{f}=v / \lambda_{0}$ where $D_{f}$ is the Doppler shift, $v$ is the relative speed of the transceivers and $\lambda_{0}$ is the signal wavelength. The Doppler shift, for instance, 


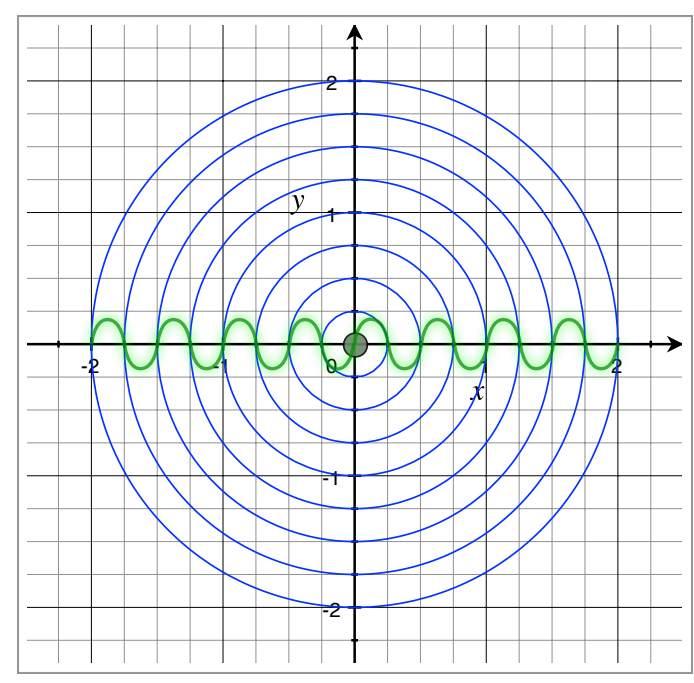

(a) Static transmitter

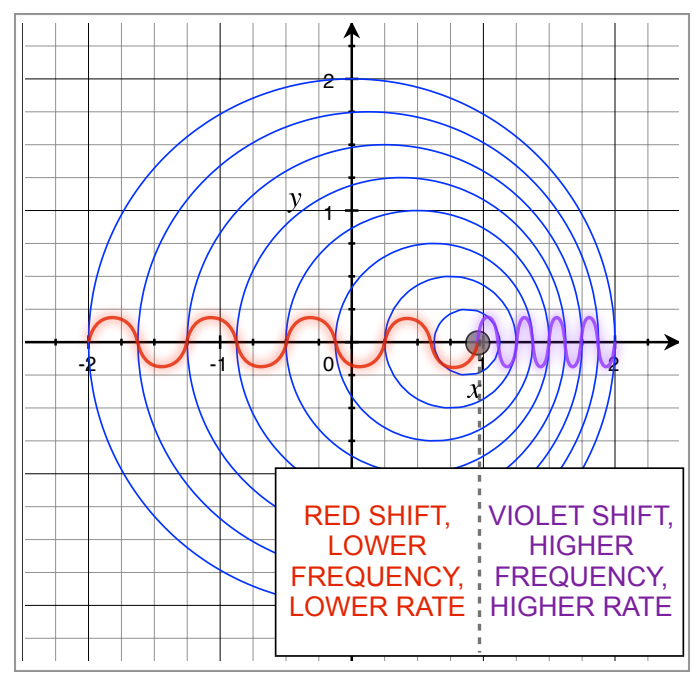

(b) Moving transmitter

Figure 2.10: Doppler effect

of a $5 \mathrm{GHz}$ signal which endpoints are moving relatively at a speed of $300 \mathrm{Km} / \mathrm{h}$ is $1389 \mathrm{~Hz}$. In the practice, it is preferable the carrier bandwidth to be much greater than the maximum Doppler shift.

\subsubsection{Summary of wireless communications challenges}

Various characteristics of wireless channels have been introduced in the previous subsections. Multipath propagation, for instance, creates ISI that becomes significative in faster transmissions. "There are many system-level approaches that mitigate the negative effects of channel fading by implementing channel reinforcing techniques in both the transmitter and receiver" [6]. Some of these techniques take place in the physical layer. Some others in upper layers - for instance, error detection and error correction.

"Adaptive modulation and coding (AMC) is a technique that adjusts the transmission rate based on the current channel condition. If the channel is in good condition, the transmitter increases the transmission rate by using higher order modulation and codification" [6]. Some systems dynamically assign higher speed rates to certain subcarriers depending on the channel conditions at particular frequencies. 
In general, the system specifications are designed to reinforce the transmission endurance in wireless channels. For instance, due to the fact that wide band carriers suffer from frequency distortion, some transmissions divide the carrier in multiple narrow channels. This divides the total bandwidth into smaller pieces of narrow band subcarriers. The subcarriers are less sensitive to frequency selective channels. Similarly, the signal period is adjusted so that is many times longer than the maximum time delay $\tau_{\max }$. This helps the receiver detect and remove the part of the carrier that contains multipath ISI. The signal period still needs to be short enough to reduce the effects of time selective channels. In the practice, careful consideration is placed during the design process depending on the environmental conditions for which the communications system is intended [35].

The aforementioned techniques are used in the radio architectures of spectrally efficient systems which architectures are described in the following Chapters. 


\section{Chapter 3}

\section{Related work}

This Chapter provides details about systems that are comparable with SSDM. Section 3.1 covers OFDM with an implementation example. Meanwhile, section 3.2 introduces several methods with higher spectral efficiency. These methods, along with OFDM, are compared with SSDM later in Chapter 7.

\subsection{OFDM}

This section introduces the aspects of OFDM that are relevant to SSDM. Accordingly, a performance comparison between SSDM and OFDM is discussed later in Section 7.1 on page 62 .

During the last decade, Orthogonal Frequency Division Multiplexing (OFDM) has been generally adopted in latest standards (WLAN, WiMAX, 4G IMT, DVB-T [29]) due to its satisfactory Spectral Efficiency (SE) as well as its intrinsic endurance to time dispersive and frequency selective channels. Compared with previous technologies, OFDM facilitates "delivering high data rates while being robust to varying mobility levels" [13].

OFDM is a mechanism that was created with the objective of increasing the SE compared with narrowband communications as in Frequency Division Multiplexing (FDM). Additionally, it reduces the susceptibility to frequency selective channels when compared 

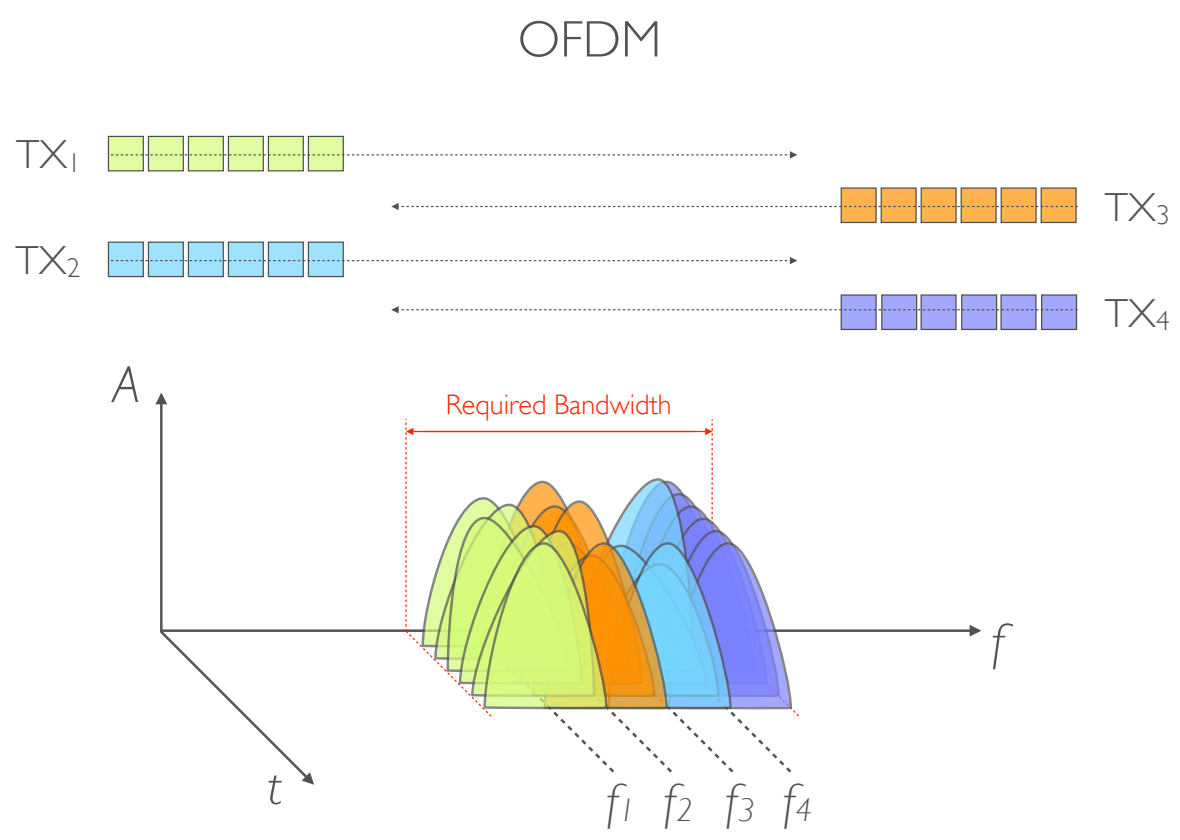

Figure 3.1: Orthogonal Frequency Division Multiplexing (OFDM)

with broadband carriers such as Time Division Multiplexing (TDM). It provides easy sectioning of the channel for multiple access. It is used in both point to point and pointmultipoint communications.

OFDM sends multiple low rate streams with RF pulses modulated with QAM at orthogonal frequencies. The orthogonality in OFDM is achieved by selecting the subcarrier frequencies so that the difference between them is a multiple of their bandwidth $\triangle f=1 / T$. Figure 3.1 shows how the spectrum of four carriers is overlapped in OFDM. The required bandwidth is $\frac{N+1}{2}$ times the bandwidth of one of the $N$ multiplexed signals.

Figure 3.2 shows the spectrum power of the subcarrier signals. As seen in Figure 3.2, the ICI is zero at the center frequency of each subcarrier $f_{i}$. The OFDM receiver is able to read the subcarriers by evaluating the spectrum value at those specific frequencies. In the example in Figure 3.2, the first frequency is $f_{1}=16 \mathrm{MHz}$ and the frequency step is $\triangle f=1 \mathrm{MHz}$. 


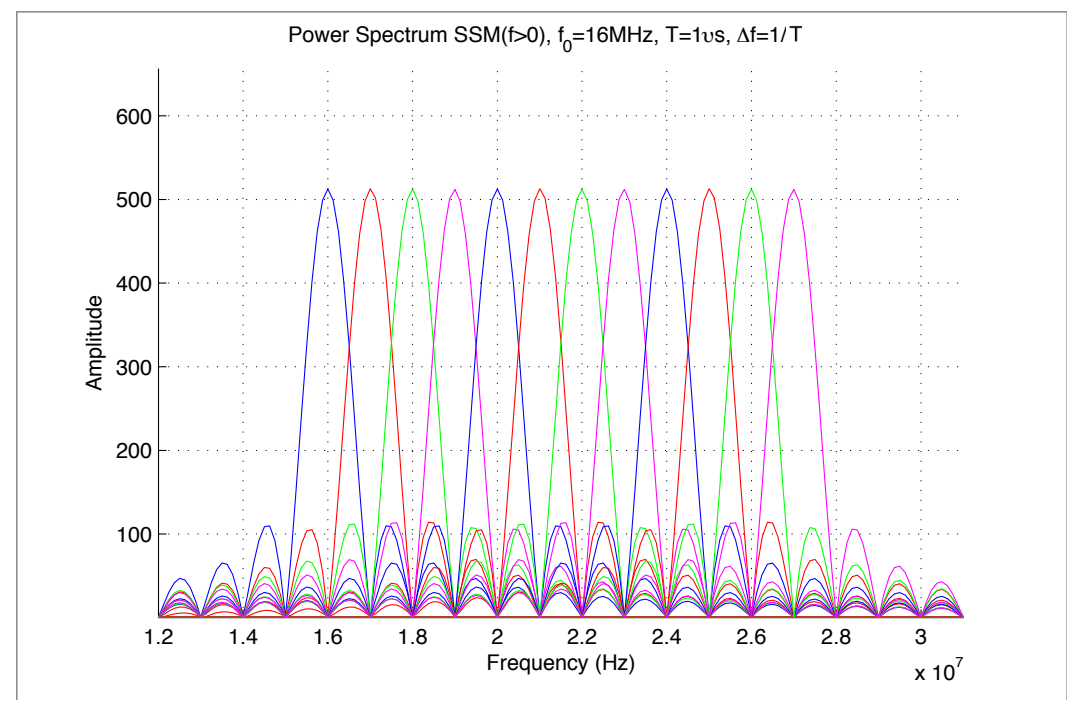

Figure 3.2: Power spectrum of 16 OFDM subcarriers.

A challenges of OFDM is high peak to average power ratio (PAPR). "A high PAPR, which results from the coherent addition of the modulated subcarriers, reduces the efficiency of the power amplifier" [38]. In Figure 3.3, severe amplitude variations are observed. Those variations appear when the signal contains two or more similar frequencies. This characteristic is typical in spectrally efficient (SE) signals.

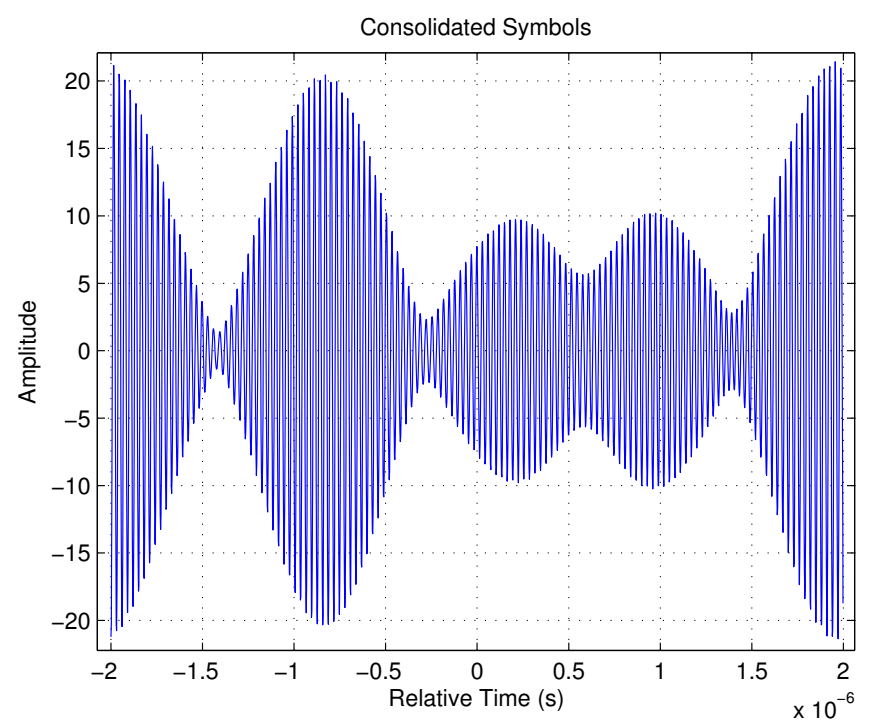

Figure 3.3: High peak to average ratio due to subcarriers overlapping in SE methods 


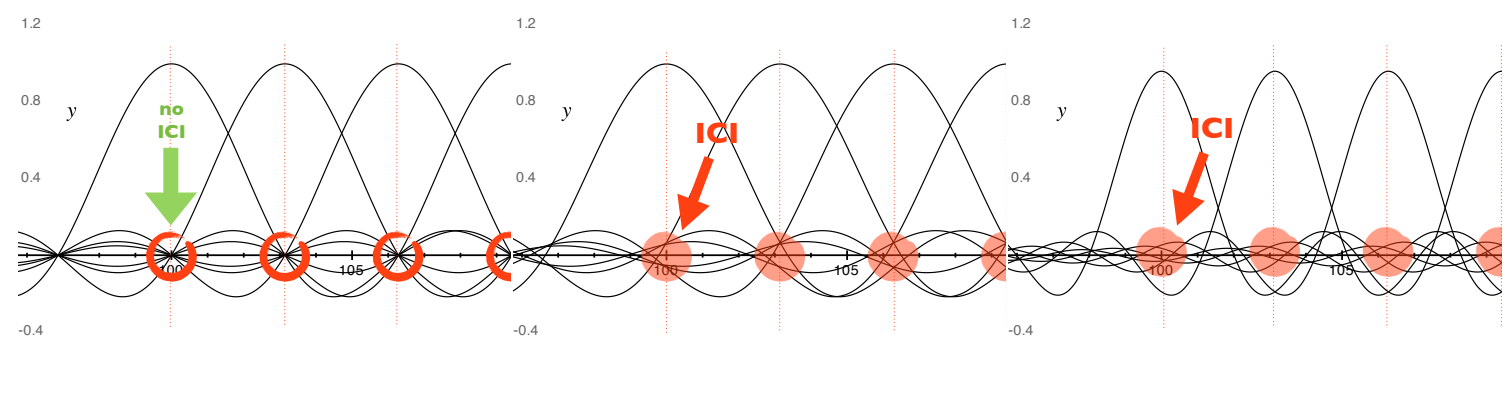

(a) ICI is null

(b) Approaching transceivers

(c) Transceivers moving away

Figure 3.4: ICI in OFDM due to Doppler effect

Another challenge of OFDM is high sensitivity to the Doppler shift. The Doppler effect widens or shrinks the spectrum of the subcarrier signals causing the loss of orthogonally. Figure 3.4 shows how relevant inter-carrier interference (ICI) from the adjacent subcarriers of (a) the original signal affects the center frequencies of the other carriers due to either (b) an approaching transmitter or (c) a transmitter moving apart.

OFDM is used in many standards such as WLAN, $4 \mathrm{G}$ and WiMAX among others. The design parameters change from one standard to another. For instance, Table 3.1 shows the design parameters of 802.11a. This standard provides speeds of up to 54Mbps using $20 \mathrm{MHz}$ of bandwidth in the $5 \mathrm{GHz}$ band. The carrier comprises of 56 subcarriers distributed in groups of 23 occupying a bandwidth of $10 \mathrm{MHz}$ each. Four of the subcarriers are pilots carriers. There is an extra virtual carrier in the center that is not modulated. Figure 3.5 shows the carrier composition of 802.11a. The purpose of both the pilot carriers and the virtual carrier is to help signal tracking.

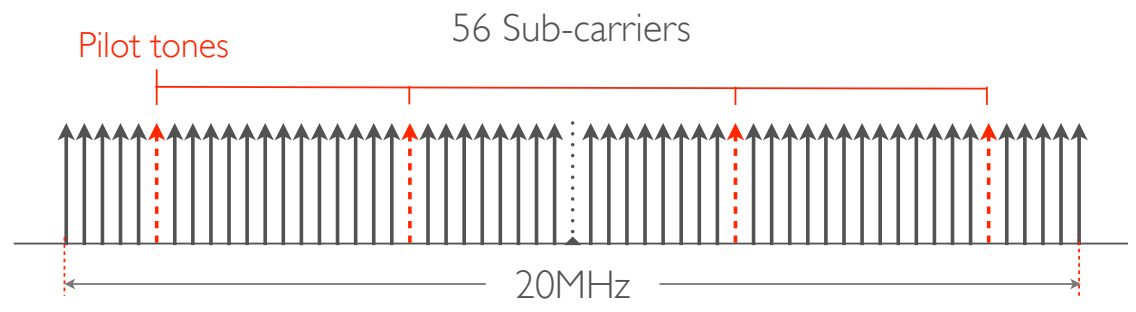

Figure 3.5: 802.11 a carrier on a $20 \mathrm{MHz}$ bandwidth 


\begin{tabular}{|l|l|}
\hline PARAMETER & VALUE \\
\hline \hline NSD : Number of data subcarriers & 48 \\
\hline NSP : Number of pilot subcarriers & 4 \\
\hline NST : Number of subcarriers, total & $52(\mathrm{NSD}+\mathrm{NSP})$ \\
\hline$\triangle f:$ Subcarrier frequency spacing & $0.3125 \mathrm{MHz}\left(=\frac{20 \mathrm{MHz}}{64}\right)$ \\
\hline TFFT : IFFT/FFT period & $3.2 \mu \mathrm{s}(1 / \triangle f)$ \\
\hline TPREAMBLE : PLCP preamble duration & $16 \mu \mathrm{s}(\mathrm{TSHORT}+\mathrm{TLONG})$ \\
\hline TSIGNAL : Duration of the SIGNAL BPSK-OFDM symbol & $4.0 \mu \mathrm{s}(\mathrm{TGI}+\mathrm{TFFT})$ \\
\hline TGI : GI duration & $0.8 \mu \mathrm{s}(T F F T / 4)$ \\
\hline TGI2 : Training symbol GI duration & $1.6 \mu \mathrm{s}(T F F T / 2)$ \\
\hline TSYM : Symbol interval & $4 \mu \mathrm{s}(\mathrm{TGI}+\mathrm{TFFT})$ \\
\hline TSHORT : Short training sequence duration & $8 \mu \mathrm{s}(10 \times T F F T / 4)$ \\
\hline TLONG : Long training sequence duration & $8 \mu \mathrm{s}(\mathrm{TGI} 2+2 \times \mathrm{TFFT})$ \\
\hline
\end{tabular}

Table 3.1: 802.11a system parameters

A possible implementation of the OFDM transmitter comprises of a serial to parallel converter followed by a QAM modulation block per subcarrier ${ }^{1}$. This configuration is shown in Figure 3.6. After mapping, the modulation constants $Q_{i}$ are delivered to an inverse fast Fourier transform (IFFT) block. The block includes extra inputs to generate a cyclic prefix. The cyclic prefix is a copy of part of the information in order to make the symbol longer. The extra information is used as a guard band against multi-path propagation (as seen in Section 2.2.1 on page 13 ).

The output of the IFFT block in Figure 3.6 is serialized somewhere in the gray block. The resultant signal is a type of pulse code modulation (PCM) that goes to a digital to analog converter (DAC). The signal is up-converted, filtered and amplified for transmission. Although variations of this transmitter exist $[38,13,4]$, they all share common components. The common components are a serial to parallel converter, constellation mappers, an inverse Fourier transform block and a DAC.

An OFDM receiver runs the inverse process as in Figure 3.7. After filtering and amplification, the signal is passed through an analog to digital converter (ADC). A signal tracking block detects the symbol period and removes the $\mathrm{CP}$. The information is arranged in parallel, equalized and processed by a fast Fourier transform (FFT) block. The results

\footnotetext{
${ }^{1}$ Some transmitters have these two stages reversed counting on a single constellation mapper.
} 


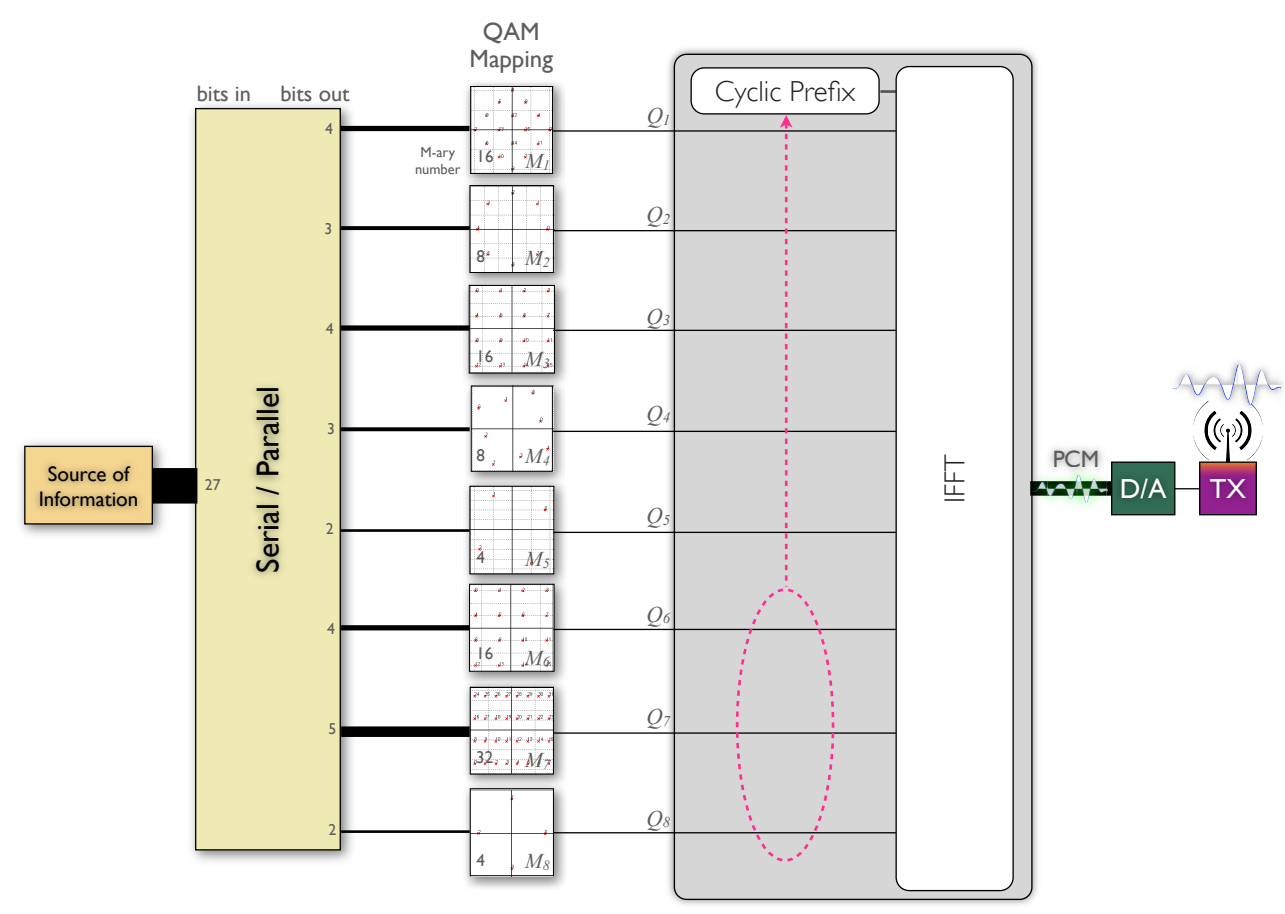

Figure 3.6: OFDM transmitter

are processed by a classifier and finally by a bit assembler that puts the information back together into digital form ${ }^{2}$.

In the practice, am OFDM carrier could be formed by a few to thousands of consecutive subcarriers. Not all of them are modulated, some are either assigned as pilot tones or not modulated at all to facilitate signal tracking ${ }^{3}$. The bit rate and the number of subcarriers used in practice depend on the specific standard.

Figure 3.8 shows the timing of the OFDM transmission. The first part of the symbols is the guard time. This part is discarded by the receiver because it probably contains ISI due to multipath propagation. In 802.11 a the guard time or $\mathrm{CP}$ is $0.8 \mu \mathrm{s}$ which is greater than $\tau_{\text {max }}$ and a symbol time of $3.2 \mu \mathrm{s}$.

In sum, OFDM is a convenient system because its subcarrier channels are orthogonal. This provides a great advantage when the receiver reads the signals at the subcarrier

\footnotetext{
${ }^{2}$ Some receivers have a parallel to serial converter at the output of the FFT block connected to a single serial classifier.

${ }^{3}$ OFDM receivers use signal tracking to determine the limits of a symbol. This provides information to the receiver about which part of the carrier contains ISI and which part is useful for demodulation.
} 


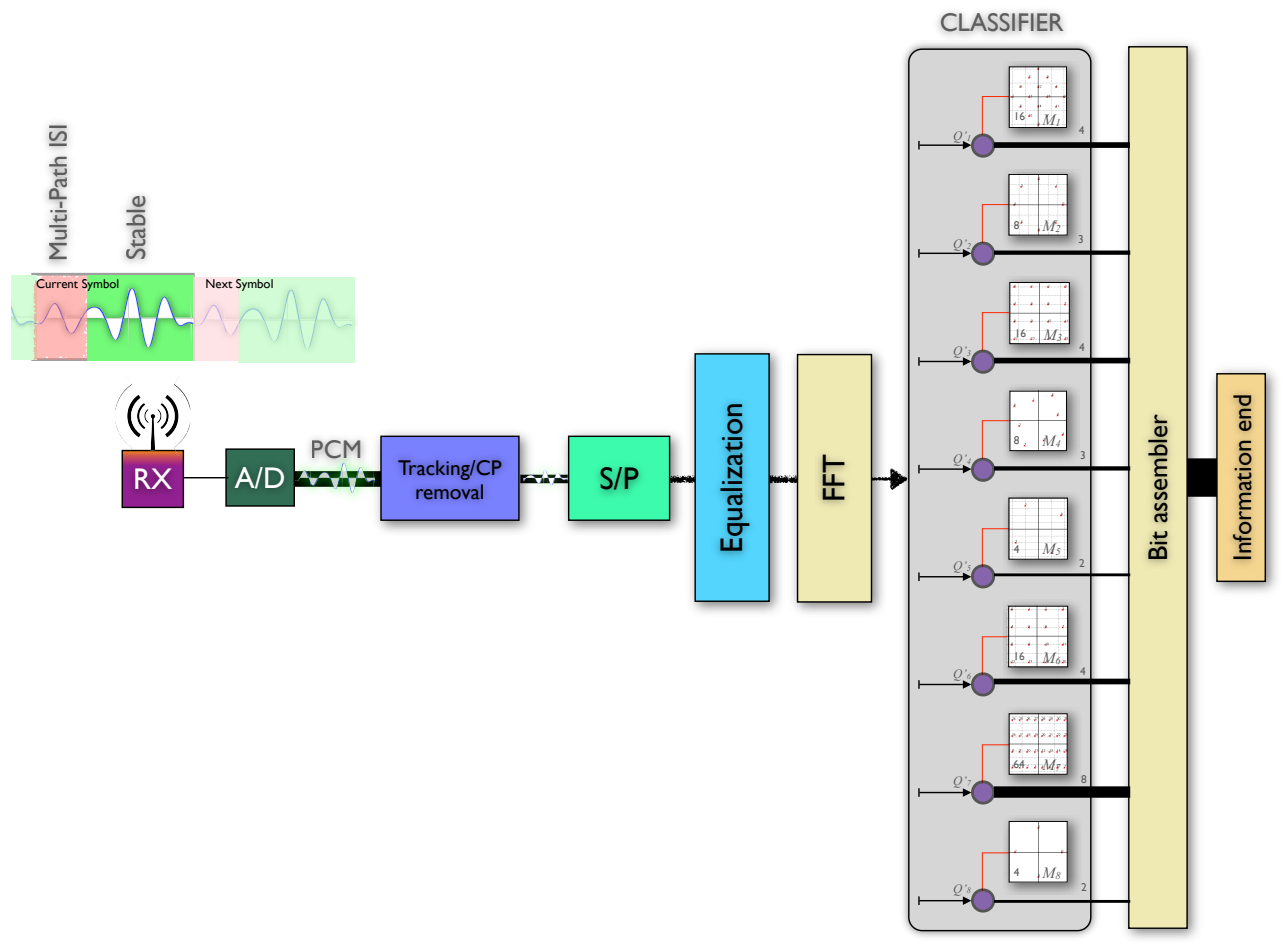

Figure 3.7: OFDM receiver

frequencies because no other subcarriers interfere. No having to deal with ICI, OFDM transceivers are simple. Besides that, the system parameters used to develop OFDM standards offer endurance and reliability of the signal in the wireless channel.

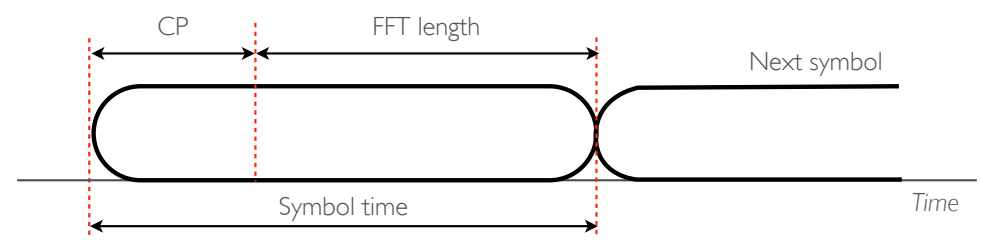

Figure 3.8: Timing in OFDM 


\subsection{Spectrally efficient (SE) methods}

While the orthogonallity idea in OFDM is an advantage, it also is a limitation. The spectrum efficiency is constrained when subcarriers are fixed at the orthogonallity norm. That constraint becomes problematic when the spectrum is limited. The following sections provide a review of the ongoing research in techniques with relaxation of the orthogonallity condition. In those techniques, the distance between subcarrier frequencies is less than orthogonal $\triangle f<1 / T$. For analysis aims, this distance is measured by the normalized subcarrier separation defined by

$$
\alpha \triangleq \triangle f \cdot T
$$

which is equal to 1 in OFDM.

Basic systems for non orthogonal communications start early in 1966 [17]. In principle, these systems were limited to two narrow carriers. Since then, diverse publications have been made regarding overlapping carriers. The majority of these publications have to do with the receiver that is the main problem. More details about these receivers are summarized in Table 3.2. The authors of these technologies have named them Spectrally Efficient Frequency Division Multiplexing [25], Non OFDM [17, 3, 7, 27] and Overlapped Frequency Division Multiplexing [22] (SEFDM, NOFDM and OvFDM correspondingly). For the rest of this work, these systems are called SE systems.

\subsubsection{OvFDM, SEFDM}

OvFDM [22] and SEFDM [25] are similar systems conceived by different authors. In these systems, the subcarrier signals are ordered and overlapped with higher density than orthogonallity $\alpha<1$. Each symbol is formed by subcarriers that are spaced equally and have sinusoidal pulses with constant amplitude.

The simplified form of SEFDM and OvFDM systems is shown in Figure 3.9 [20]. Besides the detector block that is located after the FFT block in the receiver shown in Figure $3.9 \mathrm{~b}$, the only apparent difference with OFDM is the addition of zero padding at both the input and output of the Fourier transform blocks. Many publications analyze the subcarrier overlapping problem from different perspectives and plant alternative solutions 
for the detection stage. The solution implemented affects the amount of zero padding and the length of the FFT blocks used.

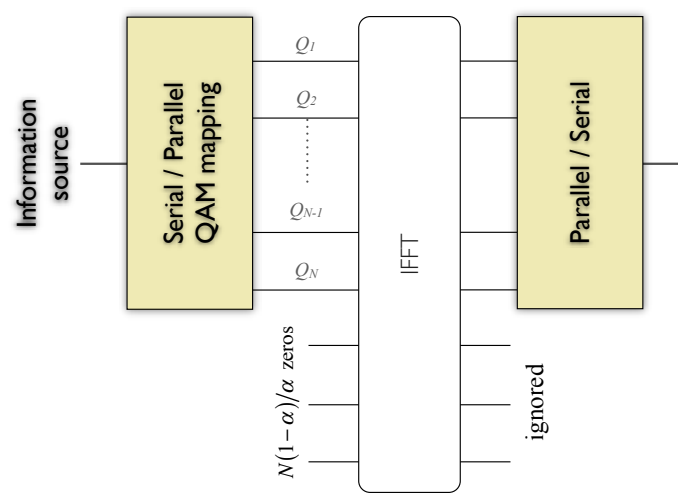

(a) Transmitter

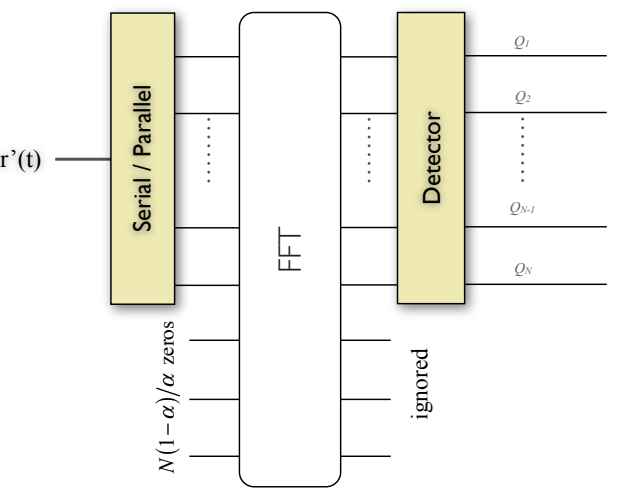

(b) Receiver

Figure 3.9: General form of SE transceivers

The purpose of the receivers is to estimate the parameters of modulation of the subcarriers $Q_{i}$. The estimation of the modulation parameters $Q_{i}$ in the receiver is called detection. In 2006, a detector for non orthogonal signals, called the sphere detector (SD), was first introduced [22]. That was the starting point for the development of most of the SE receivers available today $[2,23]$. The SD is based on two methods called zero forcing and maximum likelihood. Several modifications to the SD have been made since then combining a series of techniques to formulate new detectors. For instance, ultimate publications use heuristic transceivers with interleaving subcarriers [8].

A summary of OvFDM and SEFDM detectors is shown in Table 3.2. Apart from the mathematical approach for the detector, some papers present optimization techniques that can be implemented in any receiver such as codification [19]. Only a few publications introduce transmitters because the generation of spectrally efficient signals is generally not a problem.

All the receivers consist basically of an FFT block and a detector. The operations that take place in the detector depend on the mathematical approach that the author takes. Due to the diversity of these approaches, the only possible comparison point is the BER performance. The compiled results of comparisons between SE methods with SSDM can be seen in Section 7.3 on page 65. In some cases, the complexity of the algorithm is 


\begin{tabular}{|c|c|c|c|c|}
\hline Receiver & Publications & Approach & Iterative & Observations \\
\hline \hline $\begin{array}{c}\text { Stripe decoder for interleaved } \\
\text { overlapped SEFDM }\end{array}$ & {$[8]$} & $\begin{array}{c}\text { Interleaved carriers } \\
\text { decoded by } \\
\text { subtracting others }\end{array}$ & Yes & $\begin{array}{c}\text { Complex and computationally } \\
\text { expensive. Great results. }\end{array}$ \\
\hline $\begin{array}{c}\text { Precoded Spectrally Efficient FDM } \\
\text { System }\end{array}$ & {$[19]$} & $\begin{array}{c}\text { Two stages of } \\
\text { decodification. } \\
\text { Double FFT blocks. }\end{array}$ & No & $\begin{array}{c}\text { Requires two decoders and two } \\
\text { DFT blocks. It's }\end{array}$ \\
\hline $\begin{array}{c}\text { Truncated Singular Value } \\
\text { Decomposition (TSVD) }\end{array}$ & {$[21,18,2]$} & $\begin{array}{c}\text { Two stages: TSVD } \\
\text { and FSD }\end{array}$ & Yes & $\begin{array}{c}\text { Depends on predefined tree length. } \\
\text { Better performance than IMGS and } \\
\text { SD based systems. [2] }\end{array}$ \\
\hline $\begin{array}{c}\text { Minimum Mean Square Error } \\
\text { (MMSE) }\end{array}$ & {$[37,26]$} & $\begin{array}{c}\text { Optimizes the ML } \\
\text { the search space into } \\
\text { a multi-dimensional } \\
\text { hypersphere. }\end{array}$ & Yes & $\begin{array}{c}\text { Computational effort is random } \\
\text { and noise dependent [24]. } \\
\text { and/or the SE [18, 20, 23] }\end{array}$ \\
\hline $\begin{array}{c}\text { Sphere Decoder (SD) } \\
\text { Tries to reduce ICI }\end{array}$ & Yes & Yegrades with the increase of N \\
(FSD) & {$[20,22]$} & $\begin{array}{c}\text { Calculates minimum } \\
\text { norm for every } \\
\text { possible vector }\end{array}$ & Yes & $\begin{array}{c}\text { Brute force ML can become } \\
\text { extremely complex with the } \\
\text { increase in the system size. Low } \\
\text { performance. }\end{array}$ \\
\hline $\begin{array}{c}\text { Maximum Liked Constrained Sphere Decoder } \\
\text { (20) }\end{array}$ & Yes & $\begin{array}{c}\text { Fixed complexity compared with } \\
\text { SD. }\end{array}$ \\
\hline
\end{tabular}

Table 3.2: Overlapped frequency detectors comparison 
considered as a parameter to measure performance. However, this is typically done when the compared methods are developed by the same authors because it requires to measure the execution time of the algorithms in the same computer.

Figure3.10 shows how spectrally efficient technologies reduce the use of the spectrum in comparison with OFDM. Figure 3.10a represents the spectrum of individual subcarriers. The superposition of those subcarriers generate the SE carrier spectrum. In Figure $3.10 \mathrm{~b}$, the solid curve represents the spectrum used by OFDM with $\alpha=1$. Meanwhile, the dashed and pointed curves represent the use of the spectrum of spectral efficient signals with $\alpha=0.75$ and $\alpha=0.5$ corresponding to savings in spectrum bandwidth of $25 \%$ and $50 \%$ respectively.

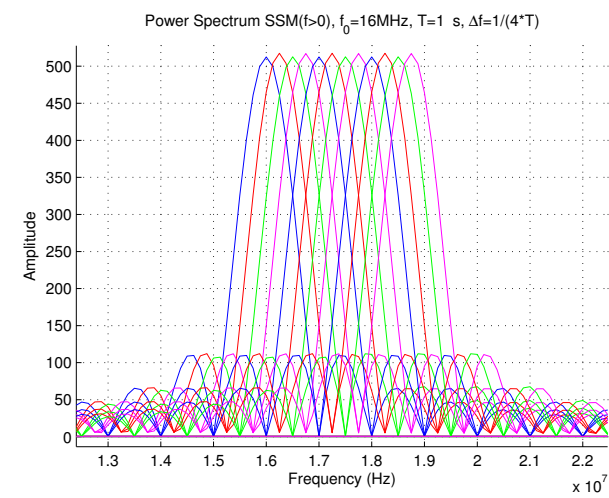

(a) 16 subcarriers in SE methods, $\alpha=0.25$

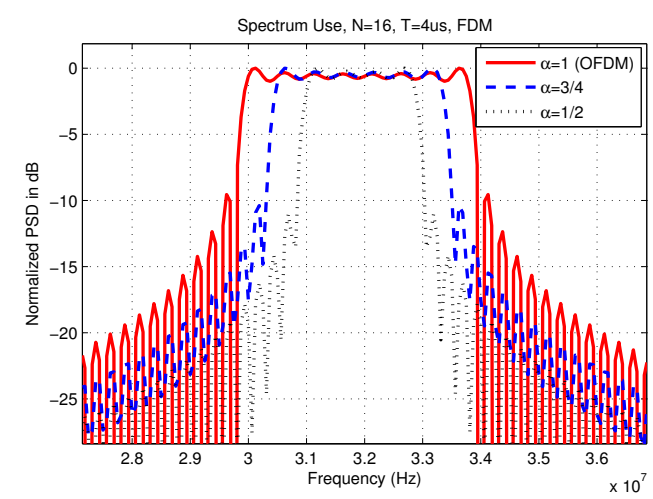

(b) SE systems compared with OFDM

Figure 3.10: Spectrum bandwidth use, OFDM vs SE methods

The following subsections provide additional information about SE detection techniques that have been benchmarked.

\subsubsection{Maximum likelihood (ML) detector}

ML detectors and their combinations [22] show lower performance than Sphere Decoders. That performance is improved by narrowing the search target in smaller sections [3]. Systems with detection based on narrow filters also use ML [28]. The main problem of ML is that the number of iterations increase exponentially with the size of the carrier $N$. 


\subsubsection{Sphere decoders (SD) [23]}

The SD is an extension of the Zero forcing (ZF) [23] detector. The ZF relays on the linear statistical model of least squares reduction. A limitation is that "it multiplies the noise vector with the inverse of the projections matrix" [18, 20, 23]. Additionally, the main matrix can become singular and consequently non-invertible. The results of this type of detector are considered suboptimal but performs well in small sized systems with high SNR [21]. SD detectors transform the ML search space into a hypersphere [20]. The SD looks for potential solutions within a determined radius in the hypersphere iteratively. In order to do that, they depart from the Cholesky decomposition of a least squares problem. The problem is that, as the SNR decreases, the SD requires an undetermined number of iterations to achieve a solution within an optimal threshold. The performance is in general better than ZF detectors.

\subsubsection{Fixed Sphere Decoder (FSD) [20]}

The iterative nature of SD makes their complexity variable depending on the noise conditions. Despite of that, the SD has been proven superior than ML detectors in terms of BER. In this sense, the authors design a fixed complexity SD. The FSD algorithm is optimized

so that the best solution is determined within a pre-specified number of iterations called the tree width. The "FSD does not guarantee an optimal solution like standard SD since it enumerates a fraction of the points within the sphere search space" [21]. However, the results show a meaningful gain in processing performance at lower levels of SNR.

\subsubsection{Interleaved overlapped SEFDM with stripe detector [8]}

This system corresponds to the evolution of many previous ones. The authors present a transmitter architecture with subcarrier interleaving and overlapping that is shown in Figure 3.11. The authors explain how the transmitter works: 


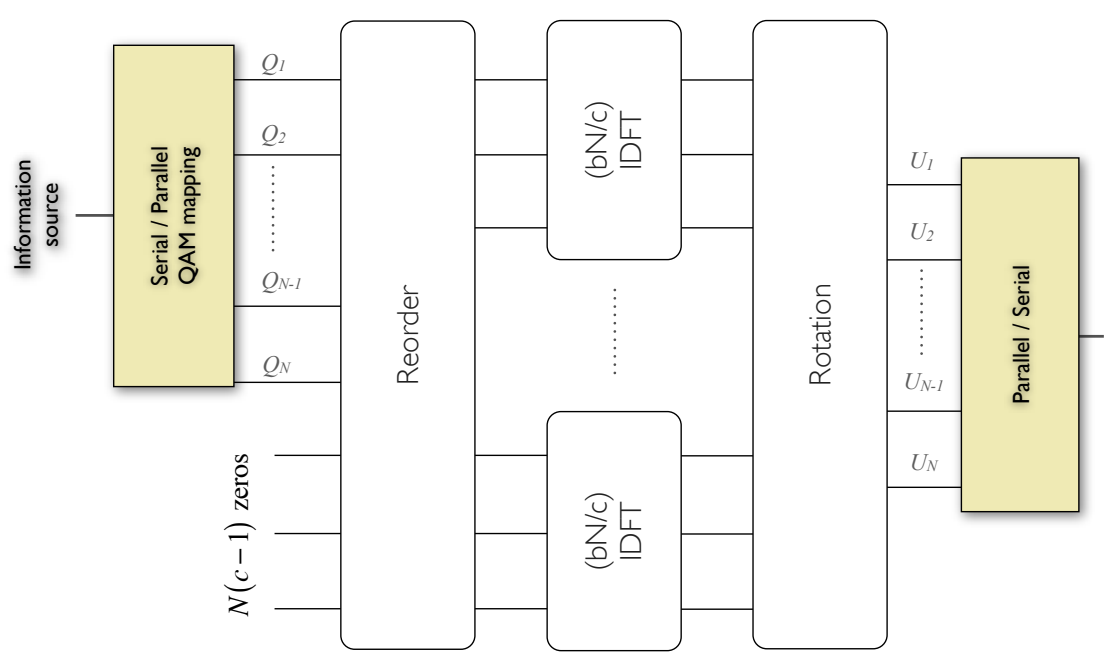

Figure 3.11: Interleaved overlapped SEFDM transmitter

"The transmitter starts by reordering the input symbols and insert zeros at appropriate locations to generate symbol vectors. These vectors are then fed into IDFT modules. The outputs of the IDFTs are then rotated using rotation matrices and combined to generate the time sampled sequence $\mathrm{U}$, which can be fed into a digital-to-analogue converter (D/A) to finally generate the continuous-time signal" [8].

For the receiver the authors claim "that the design here is heuristic, no proof of convergence is given. The justification for the design is that it is intuitive and it works in software tests". The receiver is not shown in the papers due to a typographic error but it is assumed that it has the inverse form of the transmitter. The reception process uses maximum likelihood and is described: 
"Begin with a received signal and an initial estimate that all symbols are $0+0 \mathrm{i}$ and iteratively improve the estimates by isolating the signal arising from each of sub-OFDM systems. After several iterations, the estimates converge to the correct input symbol and are eventually rounded to the closest symbol in the symbol alphabet in use ... For each of the $\mathrm{N}$ symbols calculate a 'gravitationally weighted' version ... Finally, the estimated symbols are sliced to the nearest alphabet symbol for each estimated symbol" [8].

This system is complex because it has as many Fourier transform blocks as carrier signals. Similarly, the number of iterations is proportional to $M^{N}$ being $M$ the number of elements in the constellations and $N$ the number of carriers. For instance, for a low dimension carrier with four elements in each constellation $M_{i}=4$ and five subcarriers $N=5$, the number of iterations is $4^{5}=1024$. Accordingly, the length of the Fourier transform blocks is proportional to $N \log N$. With five carriers $N=5$ there would be 5 Fourier transform blocks with length 4 . The number of transformations required is multiplied by the number of iterations, being equivalent to one transform block of length 4096.

Finally, the time required to generate the signal with the transmitter shown in Figure 3.11 is proportional to $\frac{T}{T_{S}} \log \frac{T}{T_{S}} c$ where $T$ is the signal period, $T_{S}$ the sampling period and $c$ the number of IDFT blocks.

\subsubsection{Truncated Singular Value Decomposition (TSVD) detector $[21,18]$}

This detector shows a substantial improvement in performance compared with ZF with a slight increase in complexity. The TSVD detector is similar than FSD because they both have limited complexity. It differs in that the TSVD detector uses a general reference to measure the distance of the candidates in the search space. This way the detector is not constrained to search in only a neighborhood of the hypersphere. In general, the combination of methods FSD and TSVD perform than TSVD only or FSD-ZF. As seen in the papers, there is also increased hardware complexity in order to combine these methods. 


\subsubsection{Fast Constrained Sphere Decoder (FSD) [26]}

Recent publications makes this system a candidate to replace OFDM. Similar to SSDM, it requires oversampling of the signal to perform results comparable with OFDM. Although claimed as slightly more complex than OFDM, it has a sixteen step iterative algorithm for the determination of the received symbols. This algorithm is repeated ten times per symbol decoded. Sphere detectors provide acceptable response especially in high dimensionality carriers. For smaller carriers, the iterative algorithm is quite complex compared with other methods.

\subsubsection{Precoded SEFDM detector [19]}

This SE system applies a coding strategy to simplify the detection stage. It utilizes two FFT blocks with different sizes for two different detectors. One of the detectors is ZF. The results of the ZF block feed the Precoded detector block which is based on ML. The ML mechanism relies on the output of the FFT block, the output of the ZF detector and the code itself. As shown in Figure 7.5 on page 68, it performs slightly better than other detectors requiring less SNR for a certain target BER.

\subsubsection{Non OFDM (NOFDM)}

NOFDM consists of subcarriers that overlap in both time and frequency. Figure 3.12 depicts a NOFDM carrier with arbitrary units. In Figure 3.12, every horizontal group consists of a symbol with information contained in a certain number of subcarriers. These subcarriers are overlapped at frequency steps smaller than orthogonality. In addition to that, before the transmission of the current symbol ends, the transmission of the next symbol have already started. Different than SSDM, OFDM and SEFDM, the subcarriers pulses have the form of a Gaussian or similar pulses [31]. The shading of Figure 3.12 depicts the signal strength of every subcarrier. In the left of the figure, every subcarrier has been framed in a box. At the right, the time representation of the NOFDM carrier shows how the symbols are overlapped.

Relevant studies are limited to detection and do not consider multipath channels. The detection is complex due to inherent ISI and ICI. Despite of the publications about these 


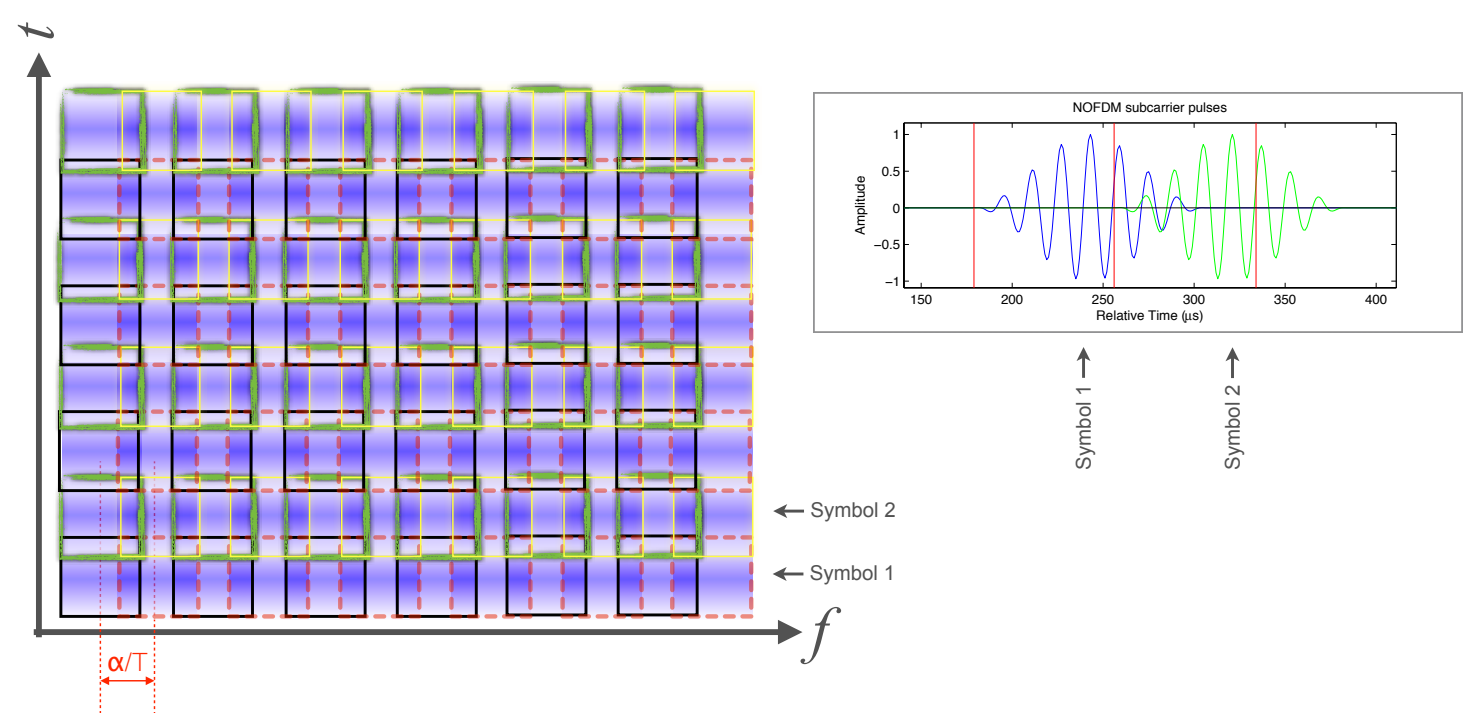

Figure 3.12: NOFDM carrier

systems, no preliminary results are shown other than optimization techniques such as precoding for bit and power allocation [33, 27].

OFDM, SEFDM and SSDM are different than NOFDM because their carriers do not overlap in time. This contrast is displayed in Figure 3.13. Moreover, there is the insertion of a cyclic postfix. NOFDM, in contrast, does not allocate a guard band to prevent multipath ISI what makes the detector extremely complex. SSDM and NOFDM, however, are similar in that they can both use any pulse shape. This is not true in OFDM and SEFDM because those systems use basically flat RF pulses.

\subsubsection{Other systems}

There are detectors based on the idea that the combination of the incoming frequencies produce a unique signature of the carrier [28]. However, these detectors use spreading codes and require an indefinite number of matched filters. This makes the communication system complex and inflexible for carrier management. Also, the detector depends on decoding the signal in a one to one relation on a discrimination tree that grows exponentially with the number of carriers so the error does. The size of the possibilities tree is $\prod_{i=1}^{N} 2^{M_{i}}$ where $N$ is the number of subcarriers and $M$ is the order of the constellation of the subcarrier $i$. 


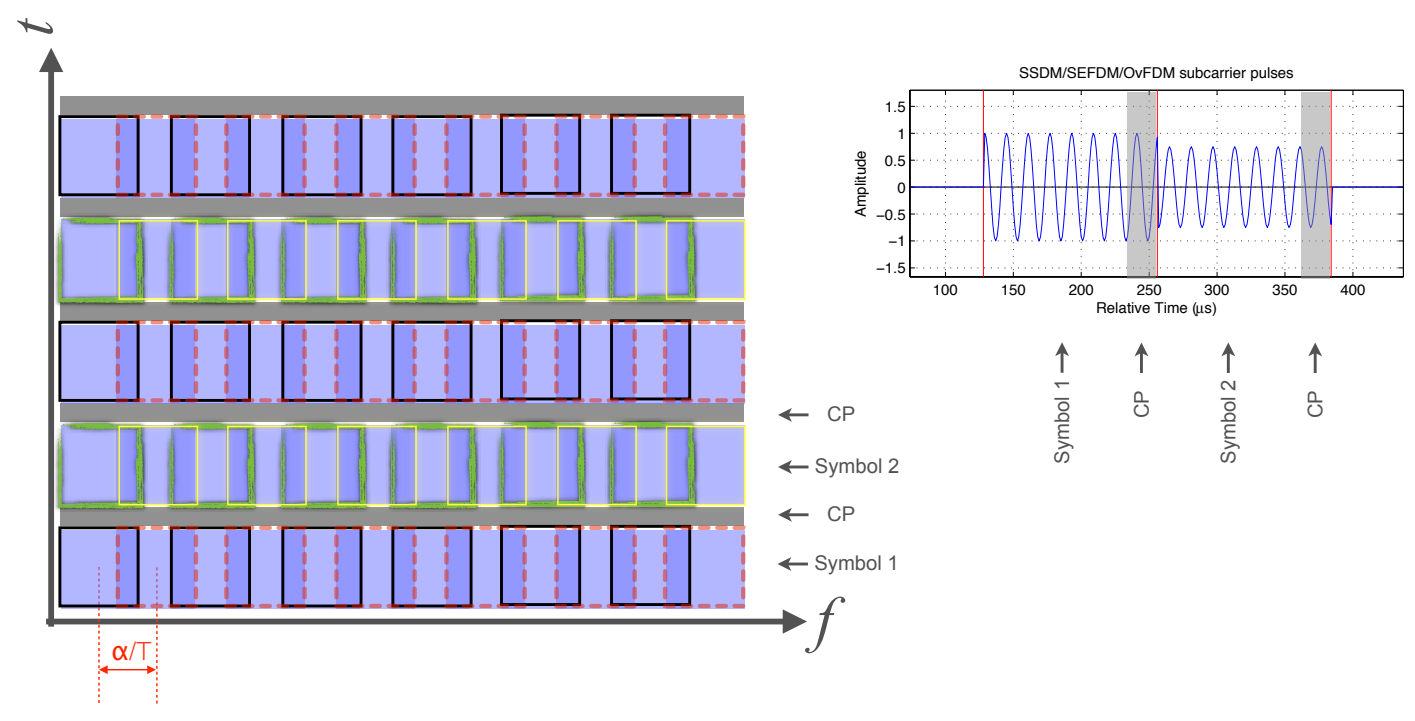

Figure 3.13: SEFDM/OvFDM/SSDM carrier

Other systems have also been called NOFDM [7]. However, they have to do with medium access control (MAC) rather than with multiplexing. MAC is a methodology for multiple devices to share a unique medium which is not relevant to this research.

Similarly, there is a field of investigation focused on obtaining higher SE by using Multiple Input Multiple Output (MIMO) [42, 39]. These systems save spectrum by taking advantage of path diversity with multiple antennas. They have to do more with multiplexing by spatial division than by frequency division which is outside of the scope of this thesis. 


\section{Chapter 4}

\section{SSDM}

In this Chapter, the SSDM transceiver is developed upon mathematical analysis. After determining the scope of the problem in Section 4.1, the basic equations of the SSDM carrier are analyzed in detail for uniform pulse shapes in Section 4.2. This section includes discrete equations that lead to the efficient equations for the SSDM transceiver. Finally, Section 4.2 also introduces briefly the concept of SSDM with non uniform pulse shapes such as Hanning pulses and uneven subcarrier frequencies.

\subsection{Scope}

The idea of SSDM encompasses many previous systems that aim for high spectral efficiency (SE). In fact, according to the parameters of the SSDM system, it can resemble one or more of these previous systems. Figure 4.1 shows the extent of SSDM among the other transmission systems.

SSDM encompasses multicarrier systems with uniform or non-uniform carriers. To prevent multipath propagation ISI, there is no time overlapping of the SSDM symbols (as in NOFDM in Figure 3.12 on page 33). The idea of SSDM is not only decoding OFDM or SEFDM/OvFDM carriers with high density, it is decoding any type of signals where the subcarriers have a unique and linear independent shape.

Except for discussions about cyclic redundancy for multi-path propagation, other methods that belong to the physical layer, such as signal tracking or equalization, are not part 


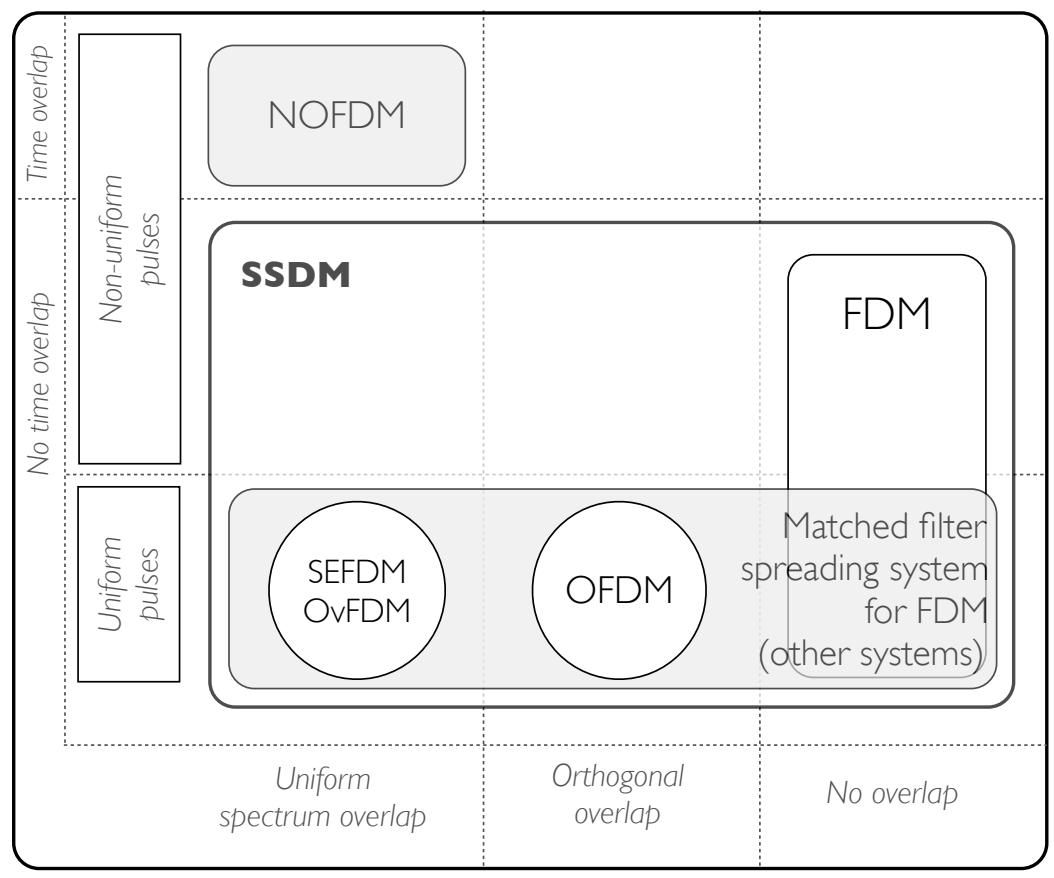

Figure 4.1: SSDM and other SE systems

of the analysis. Similarly, the SSDM system is single input single output (SISO). Multiple input multiple output (MIMO) systems [39] that count with multiple antennas and are not in the scope of this thesis.

With those considerations, the mathematics fundaments for the SSDM transceiver are formulated and analyzed. The first case developed is when all the signals have a uniform spectrum as in OFDM and SEFDM/OvFDM. The other case is a multicarrier formed by subcarriers with non standard waveforms such as Hanning pulses. Similarly, an example of a carrier with non uniform frequency steps is included. The fundamental equations for the SSDM transceivers are derived and simplified accordingly.

\subsection{Theoretical analysis}

Similar to OFDM, SDDM is a digital multicarrier able to spread high bit rate streams into lower rate subcarriers. Low rate signals are narrowband and therefore less sensitive to frequency selective channels. Besides that, the techniques used to give the SSDM carrier 
a certain endurance in the wireless channel can be taken from OFDM. For instance, the symbol time $T$ can be extended to include a guard interval (CP) to prevent multipath propagation ISI as seen in Figure 3.8 on page 24. Finally, in SSDM the subcarriers pulses are not necessarily sinusoidal nor orthogonal. SSDM can use any sub-carrier overlapping and type of pulse. OFDM, SEFDM and OvFDM are particular cases of SSDM. To compare the SSDM with the aforementioned systems, the SSDM is initially developed using sinusoidal subcarrier signals.

\subsubsection{Sub-carriers with uniform standard pulses}

An SSDM carrier comprises of $N$ subcarriers with arbitrary pulse shapes. The only condition is that the signals for different subcarriers need to be linearly independent. A formula derivation to for the SSDM transceiver can be done upon the selection of the pulse-set also called the basis signals. In this section, the formula is derived assuming standard RF pulses based on pure frequencies.

Let the SSDM carrier be encompassed of subcarriers at frequencies $f_{i}$, where $i$ is the number of subcarrier $0, \ldots, N-1$, with average separation $\triangle f=\frac{f_{N-1}-f_{0}}{N-1}$ or $\alpha=\triangle f$. $T$ where $\alpha$ is the normalized subcarrier separation. If using standard RF pulses for the subcarriers $r_{i}(t)=\cos \left(2 \pi f_{i}\right)$, the continuous function of the signals is given by

$$
s_{i}(t)=\left\{\begin{array}{cc}
A_{i} \cos \left(2 \pi f_{i} t+\phi_{i}\right) & -T / 2 \leq t \leq T / 2 \\
0 & \text { otherwise }
\end{array}\right.
$$

during the symbol period $T$, where $A_{i}$ and $\phi_{i}$ are the modulation parameters from the QAM constellation map $Q_{i}$. The constellation parameters represent the symbol sent on the subcarrier $s_{i}(t)$. The composite SSDM carrier signal responds to the linear superposition of the children

$$
s(t)=\sum_{i=1}^{N} s_{i}(t)
$$


Due to the linearity property of the Fourier transform ${ }^{1}$,

$$
\begin{aligned}
\mathscr{F}\{s(t)\} & =\mathscr{F}\left\{\sum_{i=1}^{N} s_{i}(t)\right\} \\
& =\sum_{i=1}^{N} \mathscr{F}\left\{s_{i}(t)\right\} \\
S(\omega) & =\sum_{i=1}^{N} S_{i}(\omega) .
\end{aligned}
$$

But $S_{i}(\omega)$ is given by

$$
\begin{aligned}
S_{i}(\omega)= & \int_{-\infty}^{\infty} s_{i}(t) e^{-j \omega t} d t \\
= & \int_{-T / 2}^{T / 2} A_{i} \cos \left(\omega_{i} t+\phi_{i}\right) e^{-j \omega t} d t \\
= & A_{i} \int_{-T / 2}^{T / 2} \frac{e^{j\left(\omega_{i} t+\phi_{i}\right)}+e^{-j\left(\omega_{i} t+\phi_{i}\right)}}{2} e^{-j \omega t} d t \\
= & A_{i} e^{j \phi_{i}} \frac{\sin \left[\left(\omega_{i}-\omega\right) T / 2\right]}{\omega_{i}-\omega}+A_{i} e^{-j \phi_{i}} \frac{\sin \left[\left(\omega_{i}+\omega\right) T / 2\right]}{\omega_{i}+\omega} \\
= & A_{i} \cos \phi_{i} \cdot\left(\frac{\sin \left[\left(\omega_{i}-\omega\right) T / 2\right]}{\omega_{i}-\omega}+\frac{\sin \left[\left(\omega_{i}+\omega\right) T / 2\right]}{\omega_{i}+\omega}\right) \\
& +j A_{i} \sin \phi_{i} \cdot\left(\frac{\sin \left[\left(\omega_{i}-\omega\right) T / 2\right]}{\omega_{i}-\omega}-\frac{\sin \left[\left(\omega_{i}+\omega\right) T / 2\right]}{\omega_{i}+\omega}\right) \\
\Rightarrow S_{i}(\omega)= & A_{R_{i}} B_{R}\left(\omega_{i}, \omega\right)+j A_{I_{i}} B_{I}\left(\omega_{i}, \omega\right)
\end{aligned}
$$

where $A_{R_{i}}=A_{i} \cos \left(\phi_{i}\right), A_{I_{i}}=A_{i} \sin \left(\phi_{i}\right)$ and

$$
\begin{aligned}
B_{R}\left(\omega_{i}, \omega\right) & =\frac{\sin \left[\left(\omega_{i}-\omega\right)^{T / 2}\right]}{\omega_{i}-\omega}+\frac{\sin \left[\left(\omega_{i}+\omega\right)^{T / 2}\right]}{\omega_{i}+\omega} \\
B_{I}\left(\omega_{i}, \omega\right) & =\frac{\sin \left[\left(\omega_{i}-\omega\right)^{T} / 2\right]}{\omega_{i}-\omega}-\frac{\sin \left[\left(\omega_{i}+\omega\right)^{T} / 2\right]}{\omega_{i}+\omega}
\end{aligned}
$$

\footnotetext{
${ }^{1}$ For simplicity and clarity, $\omega$ is used instead of $2 \pi f$.
} 


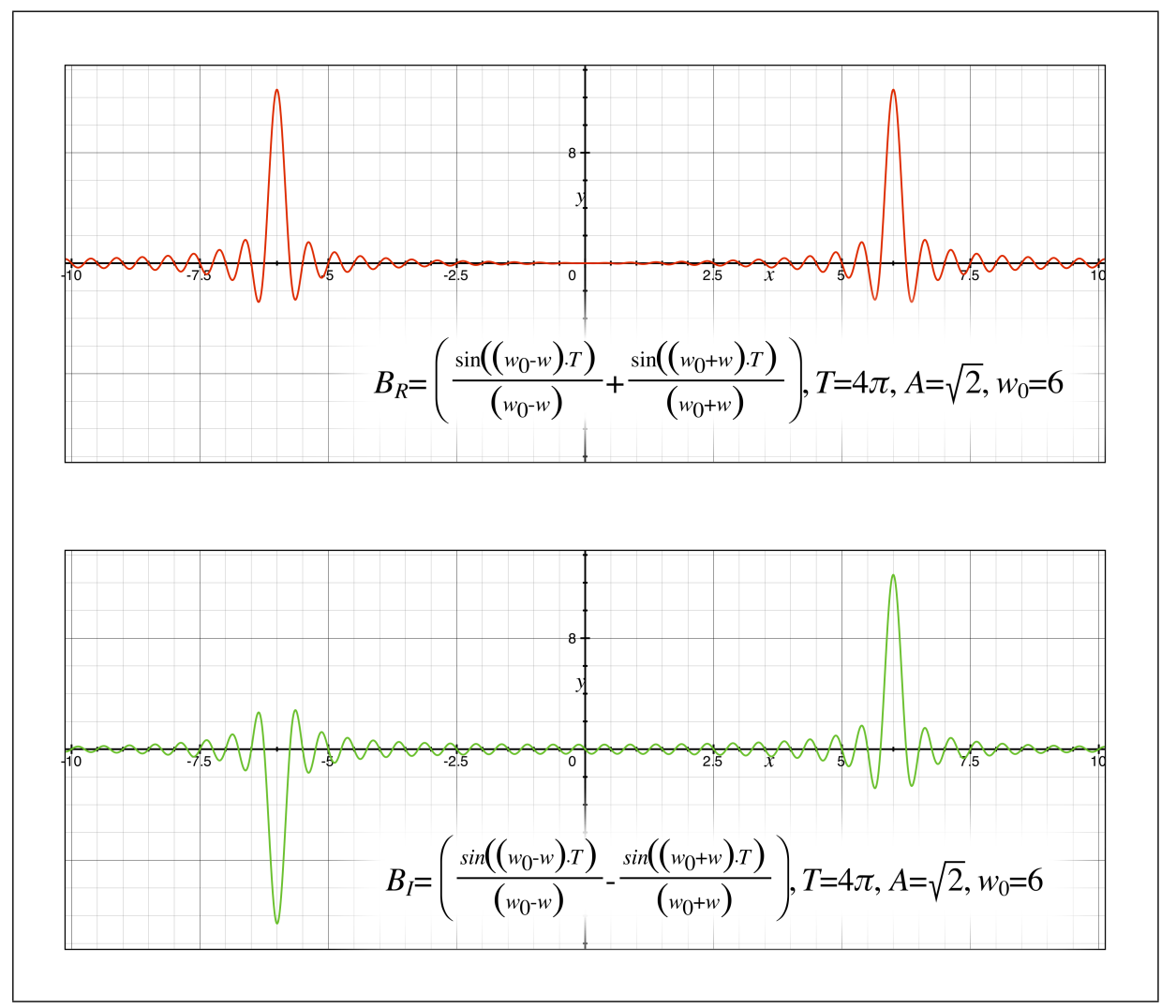

Figure 4.2: Normalized components of the spectral shape of a subcarrier

The functions $B_{R}\left(\omega_{i}, \omega\right)$ and $B_{I}\left(\omega_{i}, \omega\right)$ in Eq. 4.4 are both real and correspond to the normalized real and imaginary components of the spectrum of subcarrier $i$. These sync-like functions can be seen in Figure 4.2 for a specific $T$ and $\omega_{i}$. As expected according to the properties of the Fourier transform, the real component $B_{R}\left(\omega_{i}, \omega\right)$ presents even symmetry while the imaginary component $B_{I}\left(\omega_{i}, \omega\right)$ is odd. The weighted complex combination of the functions shown in Figure 4.2 can generate the spectrum of any symbol as indicated in Eq. 4.3. The weighting factors are the elements from the QAM constellation $A_{R_{i}}$ and $A_{I_{i}}$.

Figure 4.3 shows the view of the spectral function of a subcarrier with $1=A_{R_{i}}=A_{I_{i}}$. The projection of the function in the real and imaginary planes depict the scaled functions $A_{R_{i}} B_{R}\left(\omega_{i}, \omega\right)$ and $A_{I_{i}} B_{I}\left(\omega_{i}, \omega\right)$. The main loop of these functions have a width of $1 / T$. Another observation is that, as represented in Figure 4.3, the phase of the function varies from $-\phi$ to $+\phi$ sweeping from $-\omega_{i}$ to $+\omega_{i}$. In other words, $\phi$ is not constant in any part 


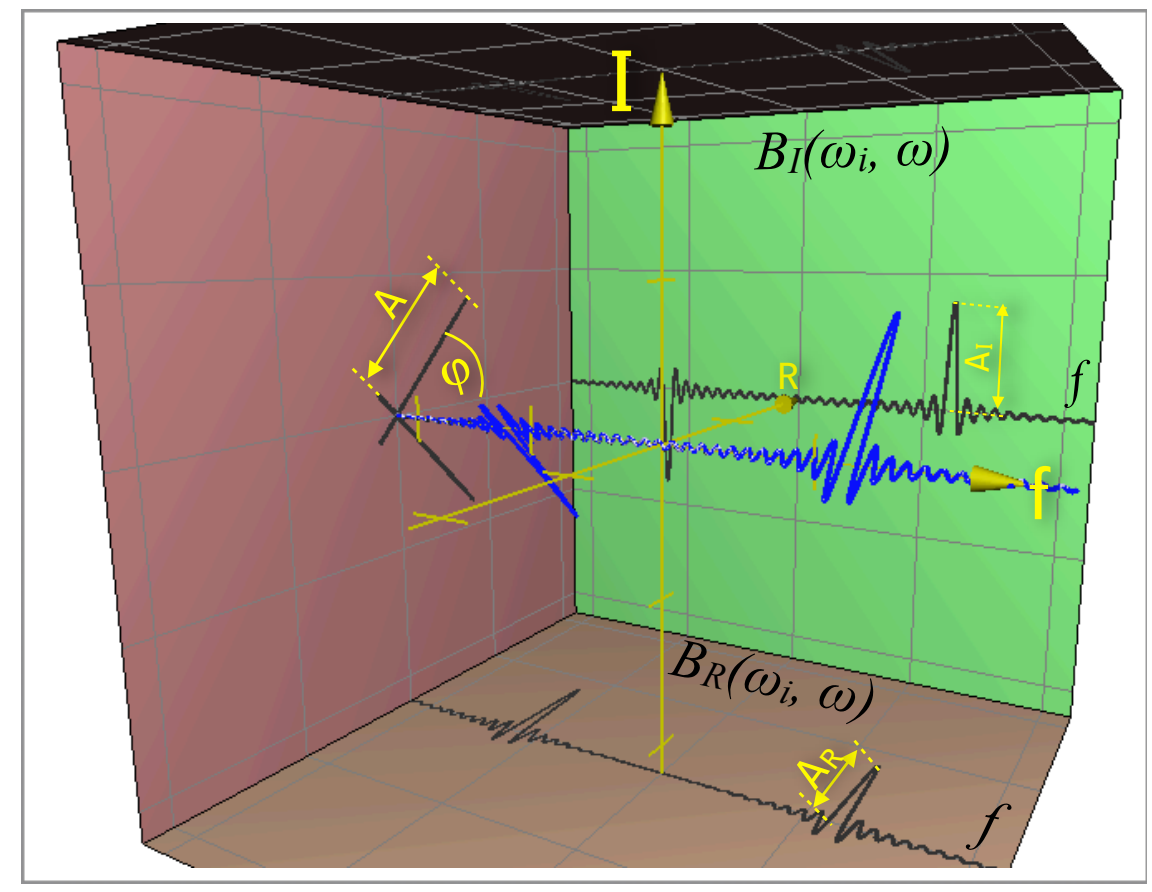

Figure 4.3: Complex spectral shape of a subcarrier and its components

of the spectral function. Due to the fact that the spectral angle of every subcarrier $\angle S_{i}(\omega)$ varies with $\omega$ and that the composite spectrum is not orthogonal, it is complex to formulate a system of linear equations to model the composite spectrum. Therefore, an approximation is made later to simplify the subcarrier functions in Eq. 4.4.

The importance of the functions $B_{R}\left(\omega_{i}, \omega\right)$ and $B_{I}\left(\omega_{i}, \omega\right)$ comes from the fact that they are the key for both the modulation and demodulation of the SSDM carrier. In fact, the spectrum of any SSDM subcarrier can be represented as a weighted combination of these functions as in 4.3. Therefore, recalling 4.2, the composite spectrum of the SSDM carrier is

$$
S(\omega)=\sum_{i=1}^{N}\left\{A_{R_{i}} B_{R}\left(\omega_{i}, \omega\right)+j A_{I_{i}} B_{I}\left(\omega_{i}, \omega\right)\right\} .
$$

The functions in 4.4 can be further simplified by making the subcarrier frequencies $f_{i}$ considerably bigger than the symbol frequency $1 / T$ and by focusing the analysis only in the positive side of these functions. $f_{i}$ represents the distance of the main loop to the axis 


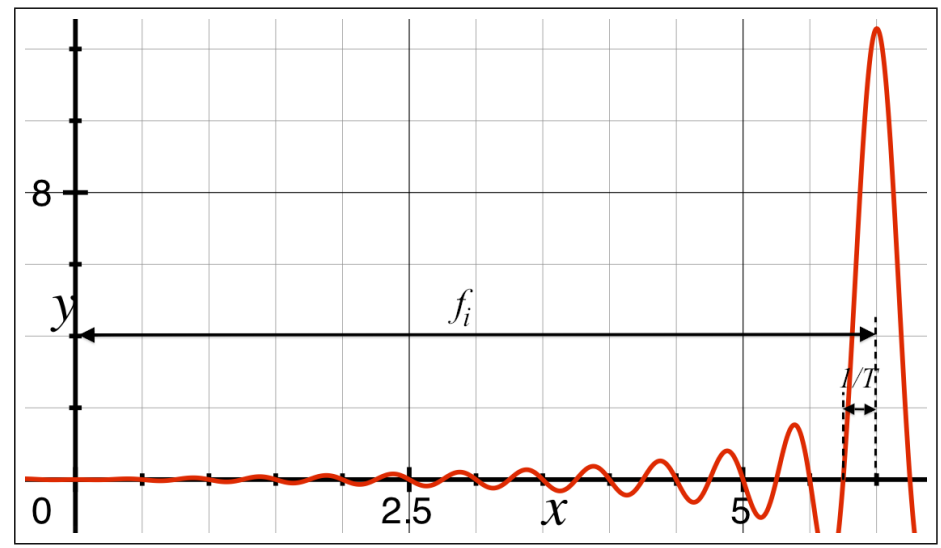

Figure 4.4: Relationship between $f_{i}$ and $1 / T$.

$f=0$. Meanwhile, $1 / T$ is the width of the main loop. Figure 4.4 shows the relationship between $f_{i}$ and $1 / T$. By making

$$
f_{i} \gg 1 / T
$$

or equivalently $\omega_{i} T \gg 1$, the main loop of the spectral function becomes considerably apart from the axis. This makes the second term of the basis functions in 4.4 negligible in the parts of the spectrum within the main loop which is nearby $\omega_{i}$. In consequence, it reduces the expression of the angle of the spectral function $\angle S_{i}(\omega)$ to a constant $\phi_{i}$.

In this sense, the second term of Eqs. 4.4 is reduced to $\lim _{\omega_{i} T \rightarrow \infty} \frac{\sin \left[\left(\omega_{i}+\omega\right)^{T / 2}\right]}{\omega_{i}+\omega}=0$ and Eqs. 4.4 to

$$
B_{R}\left(\omega_{i}, \omega\right)=B_{I}\left(\omega_{i}, \omega\right)=\frac{\sin \left[\left(\omega_{i}-\omega\right)^{T / 2}\right]}{\omega_{i}-\omega}
$$

for $\omega>0$. Therefore, Eq. 4.5 is reduced to

$$
\begin{aligned}
\lim _{\omega_{i} T \rightarrow \infty} S(\omega) & =\sum_{i=1}^{N}\left\{A_{R_{i}} \frac{\sin \left[\left(\omega_{i}-\omega\right) T / 2\right]}{\omega_{i}-\omega}+j A_{I_{i}} \frac{\sin \left[\left(\omega_{i}-\omega\right) T / 2\right]}{\omega_{i}-\omega}\right\} \\
& =\sum_{i=1}^{N}\left(A_{R_{i}}+j A_{I_{i}}\right) \frac{\sin \left[\left(\omega_{i}-\omega\right) T / 2\right]}{\omega_{i}-\omega} \\
& =\sum_{i=1}^{N} Q_{i} \frac{\sin \left[\left(\omega_{i}-\omega\right) T / 2\right]}{\omega_{i}-\omega} .
\end{aligned}
$$




\begin{tabular}{|c|c|}
\hline$\left|S_{i}(\omega)\right|$ & $\angle S_{i}(\omega)$ \\
\hline \hline$A_{i} \frac{\sin \left[\left(\omega_{i}-\omega\right)^{T / 2}\right]}{\omega_{i}-\omega}$ & $\phi_{i}$ \\
\hline
\end{tabular}

(a) Magnitude and phase

\begin{tabular}{|c|c|}
\hline $\mathfrak{N}\left\{S_{i}(\omega)\right\}$ & $\mathfrak{I}\left\{S_{i}(\omega)\right\}$ \\
\hline \hline$A_{R_{i}} \frac{\sin \left[\left(\omega_{i}-\omega\right)^{T / 2}\right]}{\omega_{i}-\omega}$ & $A_{I_{i}} \frac{\sin \left[\left(\omega_{i}-\omega\right)^{T / 2}\right]}{\omega_{i}-\omega}$ \\
\hline
\end{tabular}

(b) Real and imaginary

Table 4.1: Components of an SSDM subcarrier spectra

where $Q_{i}$ is the modulating QAM complex constant $Q_{i}=A_{i} e^{j \phi_{i}}$ such that $\left|Q_{i}\right|=A_{i}$ and $\angle Q_{i}=\phi_{i}$. The resultant spectral components of the individual subcarriers are $S_{i}(\omega)=$ $Q_{i} \frac{\sin \left[\left(\omega_{i}-\omega\right)^{T} / 2\right]}{\omega_{i}-\omega}$. The related spectral components are shown in Table 4.1.

The assumption of that $\omega_{i} T \gg 1$ is consistent with systems currently used in the practice. For instance, in the worst case scenario, a carrier at a frequency as low as $600 \mathrm{MHz}$ as used in WiMax [38] may have a symbol period as small as 3.2 $\mu$ s as used in WiFi [1] which results in $\omega_{i} T=12 \times 10^{3}$. Different from other systems, this assumption forces the digital signal processing to take place not in baseband but in intermediate frequency. The existence of fast A/D converters facilitates this task and allows omitting low pass filters and often the entire up or down converting stages [40]. The simulations in this thesis work are performed using a factor of $f_{1} T=128$; being $f_{1}$ the frequency of the first SSDM subcarrier in intermediate frequency.

\subsubsection{Discrete analysis}

Continuous equations are used in analog circuits. However, for digital signal processing, discrete analysis is required. In this section, the continuous functions 4.8 and 4.5 are analyzed in a discrete form to develop the operations of both the SSDM transmitter and receiver. These equations are supported with matrix examples.

The number of samples that comprises one SSDM symbol is a countable real number equal to $T f_{S}$ where $f_{S}$ is the sampling frequency. Let $\mathbf{t}$ be a multidimensional vector made of $t_{k}=k T_{S}$ for every $k$ in $\left\{-T f_{S} / 2,-T f_{S} / 2+1, \ldots, T f_{S} / 2-1\right\}$. Similarly, in the frequency domain, let $\mathbf{f}$ be a multidimensional vector such as $f_{k}=k / T$. Hereafter, vectors are represented in bold $\mathbf{s}$ and matrices with an added bar $\overline{\mathbf{s}}$. 


\begin{tabular}{|c|c|c|}
\hline $\begin{array}{c}\text { Normalized subcarrier } \\
\text { pulse components of length } \\
T \text { in the time domain }\end{array}$ & Modulated subcarriers in the time domain & $\begin{array}{c}\text { Transmitted signal in the time } \\
\text { domain }\end{array}$ \\
\hline \hline$c_{a:} \sin \left(2 \pi f_{a} t\right), \cos \left(2 \pi f_{a} t\right)$ & $\mathbf{s}_{\mathbf{a}}\left(t_{k}\right)=A_{a_{R}} \cos \left(2 \pi f_{a} \mathbf{t}\right)-A_{a_{I}} \sin \left(2 \pi f_{a} \mathbf{t}\right)$ & \multirow{2}{*}{$\sin \left(t_{k}\right)=\mathbf{s}_{\mathbf{a}}\left(t_{k}\right)+\mathbf{s}_{\mathbf{b}}\left(t_{k}\right)+\mathbf{s}_{\mathbf{c}}\left(t_{k}\right)$} \\
\hline$c_{b:} \sin \left(2 \pi f_{b} t\right), \cos \left(2 \pi f_{b} t\right)$ & $\mathbf{s}_{\mathbf{b}}\left(t_{k}\right)=A_{b_{R}} \cos \left(2 \pi f_{b} \mathbf{t}\right)-A_{b_{I}} \sin \left(2 \pi f_{b} \mathbf{t}\right)$ & $\mathbf{s}$ \\
\hline$c_{c:} \sin \left(2 \pi f_{c} t\right), \cos \left(2 \pi f_{c} t\right)$ & $\mathbf{s}_{\mathbf{c}}\left(t_{k}\right)=A_{c_{R}} \cos \left(2 \pi f_{c} \mathbf{t}\right)-A_{c_{I}} \sin \left(2 \pi f_{c} \mathbf{t}\right)$ & \\
\hline
\end{tabular}

Table 4.2: SSDM modulation in the time domain

Thus, taking Eq. 4.1, $\overline{\mathbf{s}}\left[t_{k}\right]=\sum_{i=1}^{N} \mathbf{s}_{i}\left[t_{k}\right]$ where $\mathbf{s}_{i}\left[t_{k}\right]=\sum_{k=-T f_{S} / 2}^{T f_{S} / 2-1} A_{i} \cos \left(2 \pi f_{i} t_{k}+\phi_{i}\right)$. Similarly, Eqs. 4.2 and 4.8 become

$$
\overline{\mathbf{S}}\left[f_{k}\right]=\sum_{i=1}^{N} \mathbf{S}_{i}\left[f_{k}\right]
$$

where $\mathbf{S}_{i}\left[f_{k}\right]=\sum_{k=-T f_{S} / 2}^{T f_{S} / 2-1} Q_{i} \frac{\sin \left[\pi\left(f_{i}-f_{k}\right) T\right]}{2 \pi\left(f_{i}-f_{k}\right)}$ for $f_{k}>0$ and

$$
f_{i} \gg 1 / T
$$

Let

$$
\mathbf{R}_{i}^{\prime}\left[f_{k}\right]=\sum_{k=-T f_{S} / 2}^{T f_{S} / 2-1} \frac{\sin \left[\pi\left(f_{i}-f_{k}\right) T\right]}{2 \pi\left(f_{i}-f_{k}\right)} .
$$

Therefore, $\mathbf{S}_{i}\left[f_{k}\right]=Q_{i} \mathbf{R}_{i}^{\prime}\left[f_{k}\right]$.

\subsubsection{Matrix example for formula derivation}

Let an SSDM system with 3 subcarriers be the example. The subcarriers are named $a, b$ and $c$ and have frequencies $f_{a}, f_{b}$ and $f_{c}$. The SSDM carrier is generated as follows in Table 4.2.

The frequency representation of the SSDM carrier is $\mathbf{S}\left(f_{k}\right)=\mathbf{S}_{\mathbf{a}}\left(f_{k}\right)+\mathbf{S}_{\mathbf{b}}\left(f_{k}\right)+\mathbf{S}_{\mathbf{c}}\left(f_{k}\right)$. By expanding the real part of Eq. 4.9 (the subindex $R$ denotes the real part), 


$$
\begin{aligned}
& \mathbf{S}_{\mathbf{R a}}\left(f_{k}\right)=A_{R a}\left[\begin{array}{lllll}
\cdots & S_{R a}\left(f_{k-1}\right) & S_{R a}\left(f_{k}\right) & S_{R a}\left(f_{k+1}\right) & \cdots
\end{array}\right] \\
& \mathbf{S}_{\mathbf{R b}}\left(f_{k}\right)=A_{R b}\left[\begin{array}{lllll}
\cdots & S_{R b}\left(f_{k-1}\right) & S_{R b}\left(f_{k}\right) & S_{R b}\left(f_{k+1}\right) & \cdots
\end{array}\right] \\
& \mathbf{S}_{\mathbf{R c}}\left(f_{k}\right)=A_{R c}\left[\begin{array}{lllll}
\cdots & S_{R c}\left(f_{k-1}\right) & S_{R c}\left(f_{k}\right) & S_{R c}\left(f_{k+1}\right) & \cdots
\end{array}\right]
\end{aligned}
$$

that is equivalent to

$$
\begin{aligned}
& \mathbf{S}_{\mathbf{R b}}\left(f_{k}\right)=\left[\begin{array}{lllll}
\cdots & A_{R a} \cdot S_{R a}\left(f_{k-1}\right) & A_{R a} \cdot S_{R a}\left(f_{k}\right) & A_{R a} \cdot S_{R a}\left(f_{k+1}\right) & \cdots
\end{array}\right] \\
& \mathbf{S}_{\mathbf{R b}}\left(f_{k}\right)=\left[\begin{array}{lllll}
\cdots & A_{R b} \cdot S_{R b}\left(f_{k-1}\right) & A_{R b} \cdot S_{R b}\left(f_{k}\right) & A_{R b} \cdot S_{R b}\left(f_{k+1}\right) & \cdots
\end{array}\right] \\
& \mathbf{S}_{\mathbf{R c}}\left(f_{k}\right)=\left[\begin{array}{lllll}
\cdots & A_{R c} \cdot S_{R c}\left(f_{k-1}\right) & A_{R c} \cdot S_{R c}\left(f_{k}\right) & A_{R c} \cdot S_{R c}\left(f_{k+1}\right) & \cdots
\end{array}\right]
\end{aligned}
$$

By adding this vectors together and transposing them,

$$
\left[\begin{array}{c}
\vdots \\
S_{R}\left(f_{k+1}\right) \\
S_{R}\left(f_{k}\right) \\
S_{R}\left(f_{k-1}\right) \\
\vdots
\end{array}\right]=\left[\begin{array}{c}
\vdots \\
A_{R a} \cdot S_{R a}\left(f_{k+1}\right)+A_{R b} \cdot S_{R b}\left(f_{k+1}\right)+A_{R c} \cdot S_{R c}\left(f_{k+1}\right) \\
A_{R a} \cdot S_{R a}\left(f_{k}\right)+A_{R b} \cdot S_{R b}\left(f_{k}\right)+A_{R c} \cdot S_{R c}\left(f_{k}\right) \\
A_{R a} \cdot S_{R a}\left(f_{k-1}\right)+A_{R b} \cdot S_{R b}\left(f_{k-1}\right)+A_{R c} \cdot S_{R c}\left(f_{k-1}\right) \\
\vdots
\end{array}\right]
$$

which can be expressed as a matrix by vector multiplication as

$$
\left[\begin{array}{c}
\vdots \\
S_{R}\left(f_{k+1}\right) \\
S_{R}\left(f_{k}\right) \\
S_{R}\left(f_{k-1}\right) \\
\vdots
\end{array}\right]=\left[\begin{array}{ccc}
\vdots & \vdots & \vdots \\
S_{R a}\left(f_{k+1}\right) & S_{R b}\left(f_{k+1}\right) & S_{R c}\left(f_{k+1}\right) \\
S_{R a}\left(f_{k}\right) & S_{R b}\left(f_{k}\right) & S_{R c}\left(f_{k}\right) \\
S_{R a}\left(f_{k-1}\right) & S_{R b}\left(f_{k-1}\right) & S_{R c}\left(f_{k-1}\right) \\
\vdots & \vdots & \vdots
\end{array}\right] \cdot\left[\begin{array}{c}
A_{R a} \\
A_{R b} \\
A_{R c}
\end{array}\right]
$$

Letting this expression be $\chi_{R}=\Gamma_{R} \times \Lambda_{R}$ is convenient because: 
- $\chi_{R}$ corresponds to the spectrum of the SSDM carrier.

- The columns in the matrix $\Gamma_{R}$ contain the spectral form of one subcarrier each. Accordingly, this matrix has $N$ columns.

- $\Lambda_{R}$ contains the real part of the modulating symbols $Q_{i}$.

The analysis of the imaginary part is no different, therefore, $\chi_{I}=\Gamma_{I} \times \Lambda_{I}$. Additionally, it is known as seen in Eqs. 4.7 and 4.8 that $\Gamma_{R}=\Gamma_{I}=\Gamma$. Therefore, the complete answer $\chi=\chi_{R}+j \chi_{I}=\Gamma \times \Lambda_{R}+j \Gamma \times \Lambda_{I}=\Gamma \times\left(\Lambda_{R}+j \Lambda_{I}\right)$. However,

$$
\Lambda_{R}+j \Lambda_{I}=\left[\begin{array}{l}
Q_{a} \\
Q_{b} \\
Q_{c}
\end{array}\right]=\Lambda
$$

As a consequence,

$$
\chi=\bar{\Gamma} \times \Lambda
$$

where $\chi=\mathbf{S}\left[f_{k}\right]$ represents the SSDM carrier and $\Gamma$, called the projection matrix, corresponds to a normalized matrix of spectral shapes with $(1+j) R_{i}^{\prime}\left[f_{k}\right]$ in every column as defined in Eq. 4.11. It can be shown by reversing Eq. 4.8 that the base function that corresponds to $\mathbf{R}_{i}\left[f_{k}\right]=(1+j) R_{i}^{\prime}\left[f_{k}\right]$ in the time domain is $r_{i}(t)=\cos \left(2 \pi f_{i} t+\pi / 4\right)$. This is

$$
\bar{\Gamma}\left[f_{k}\right]=[\overbrace{\left[\begin{array}{c}
\left\{R_{1}\left(f_{k}\right)\right\}_{k=-T f_{S} / 2}^{T f_{S} / 2-1} \\
\vdots \\
(1+j) \frac{\sin \left[\pi\left(f_{1}-f_{k}\right) T\right]}{2 \pi\left(f_{1}-f_{k}\right)} \\
\vdots
\end{array}\right]}^{\{\overbrace{\left[\begin{array}{c}
(1+j) \frac{\sin \left[\pi\left(f_{2}-f_{k}\right) T\right]}{2 \pi\left(f_{2}-f_{k}\right)} \\
\vdots
\end{array}\right]}^{\left\{R_{2}\left(f_{k}\right)\right\}_{k=-T f_{S} / 2}^{T f_{S} / 2-1}}]} \cdots
$$

With this, the SSDM carrier for this example can be also generated in the frequency domain as shown in Table 4.3. 


\begin{tabular}{|c|c|c|c|c|}
\hline $\begin{array}{c}\text { Normalized subcarrier } \\
\text { pulses of length } T \text { in time } \\
\text { domain }\end{array}$ & $\begin{array}{l}\text { Spectrum of the } \\
\text { subcarrier signals }\end{array}$ & $\begin{array}{l}\text { Subcarrier signals } \\
\text { modulated in the } \\
\text { frequency domain }\end{array}$ & SSDM carrier spectrum & $\begin{array}{l}\text { Transmitted signal } \\
\text { in the time domain }\end{array}$ \\
\hline $\mathbf{r}_{\mathbf{a}}(t)=\cos \left(2 \pi f_{a} t+\pi / 4\right)$ & $\mathscr{F}\left\{\mathbf{r}_{\mathbf{a}}(t)\right\}=\mathbf{R}_{\mathbf{a}}(f)$ & $\overline{\mathbf{S}_{\mathbf{a}}(f)=Q_{a} \cdot \mathbf{R}_{\mathbf{a}}(f)}$ & \multirow{3}{*}{$\mathbf{S}(f)=\mathbf{S}_{\mathbf{a}}(f)+\mathbf{S}_{\mathbf{b}}(f)+\mathbf{S}_{\mathbf{c}}(f)$} & \multirow{3}{*}{$\mathscr{F}^{-1}\{\mathbf{S}(f)\}=\mathbf{s}(t)$} \\
\hline $\mathbf{r}_{\mathbf{b}}(t)=\cos \left(2 \pi f_{b} t+\pi / 4\right)$ & $\mathscr{F}\left\{\mathbf{r}_{\mathbf{b}}(t)\right\}=\mathbf{R}_{\mathbf{b}}(f)$ & $\mathbf{S}_{\mathbf{b}}(f)=Q_{b} \cdot \mathbf{R}_{\mathbf{b}}(f)$ & & \\
\hline $\mathbf{r}_{\mathbf{c}}(t)=\cos \left(2 \pi f_{c} t+\pi / 4\right)$ & $\mathscr{F}\left\{\mathbf{r}_{\mathbf{c}}(t)\right\}=\mathbf{R}_{\mathbf{c}}(f)$ & $\mathbf{S}_{\mathbf{c}}(f)=Q_{c} \cdot \mathbf{R}_{\mathbf{c}}(f)$ & & \\
\hline
\end{tabular}

Table 4.3: SSDM modulation in the frequency domain 
Eq. 4.13 determines the relationship between the modulating constants $\Lambda$ and the composite spectrum $\chi$. On the other hand, the opposite relationship can be derived directly. Eq. 4.13 can be written like

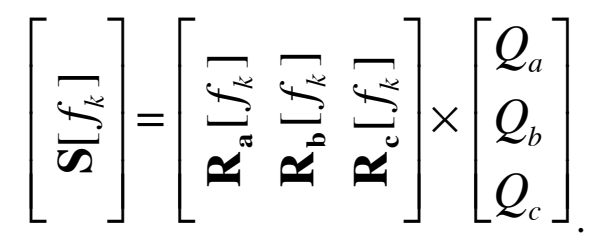

Although the length of the vectors $\mathbf{S}$ and $\mathbf{R}$ is $T / T_{S}$, only $N$ elements are required for decoding. Therefore, by selecting arbitrarily $N$ rows from $\chi$ and $\bar{\Gamma}, \bar{\Gamma}$ becomes square and this expression can be reversed to

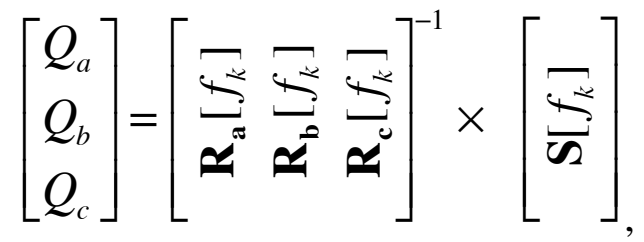

therefore,

$$
\Lambda^{\prime}=\bar{\Gamma}^{-1} \times \chi^{\prime}
$$

where $\chi^{\prime}$ represents the spectrum of the received signal $s^{\prime}(t), \Gamma^{-1}$ the inverse of the normalized functions matrix and $\Lambda^{\prime}$ a vector with the received symbols $Q_{i}^{\prime}$. The inverse matrix $\Gamma^{-1}$ can be calculated off-line and be hardcoded at the receiver.

The selection of the $N$ rows to use is a matter of convenience. In principle, the center frequencies of the carriers are preferred. However, this selection is independent from $f_{i}$ and could vary depending on the application.

This synthesized formula of SSDM is seemingly simple when compared with the detectors shown in Section 3.2. The SSDM the detector comprises of one matrix by vector multiplication of order $[\because]_{N \times N} \times[\vdots]_{N \times 1}$. Similarly, the FFT blocks do not need to be complete. Only $N$ outputs are necessary leaving the implementation to discretion of the use of Fractional Discrete Fourier Transform blocks (FrDFT) ${ }^{2}$. Finally, if the subcarrier

\footnotetext{
${ }^{2}$ The FrDFT omits computing terms that are not used at the output. Similarly, the The IFrDFT does not compute terms for inputs that are zero. More information about the FrDFT is included in Appendix A on page 74.
} 
frequencies remain constant, the most complex operations can be performed off-line and be hardcoded in both the transmitter and the receiver.

\subsubsection{Sub-carriers with non standard pulse shapes}

In Section 4.2, the formula for the SSDM transceiver was derived for standard sinusoidal RF pulses. However, that is only the punctual case of SSDM in which all the subcarriers are homogeneous and have the same spectral shape. In this section, the projection matrix is developed for the SSDM system with both Hanning-windowed pulses and heterogeneous subcarriers. The use of non standard RF pulses or windowing could have many applications: detect signals that are shifted from the expected frequency, increase the density of the subcarriers where the channel seems favorable, randomize the carrier for security purposes, reducing external interference or correcting distortion. Disregarding the preference of use, this ability brings great flexibility to the SSDM radio.

\subsubsection{Hanning RF pulse}

The spectral function in Equation 4.3 on page 38 can be replicated if a Hanning window $h(t)$ is applied to the transmitted signal. In that case:

$$
\begin{aligned}
& s(t)=\left\{\begin{array}{cc}
A \cos \left(\omega_{0} t+\phi\right) & -T / 2 \leq t \leq T / 2 \\
0 & \text { otherwise }
\end{array}\right. \\
& h(t)=\left\{\begin{array}{cc}
\frac{1}{2} \cos \left(\frac{2 \pi t}{T}\right)+\frac{1}{2} & -T / 2 \leq t \leq T / 2 \\
0 & \text { otherwise }
\end{array} .\right.
\end{aligned}
$$

Let $\omega_{s}=\frac{2 \pi}{T}$ be the frequency of the symbols, and $A_{R}=A \cos \phi$ and $A_{R}=A \sin \phi$ the real and imaginary components of $A e^{j \phi}$. The Fourier transform of $s(t) \cdot h(t)$ is $\mathscr{F}\{s(t) \cdot h(t)\}=S_{H}(\omega)$. Therefore, the spectral function for a subcarrier with frequency 
$\omega_{0}$ is:

$$
\begin{aligned}
& S_{H}(\omega)=\int_{-\infty}^{\infty} s(t) \cdot h(t) e^{-j \omega t} d t \\
& =\int_{-T / 2}^{T / 2} A \cos \left(\omega_{0} t+\phi\right)\left(\frac{1}{2} \cos \omega_{s} t+\frac{1}{2}\right) e^{-j \omega t} d t \\
& =\frac{A}{8} e^{j \phi} \int_{-T / 2}^{T / 2}\left(2 e^{j \omega_{0} t}+e^{j\left(\omega_{0}-\omega_{S}\right) \mathrm{t}}+e^{j\left(\omega_{0}+\omega_{S}\right) \mathrm{t}}\right) e^{-j \omega t} d t \\
& +\frac{A}{8} e^{-j \phi} \int_{-T / 2}^{T / 2}\left(2 e^{-j \omega_{0} t}+e^{-j\left(\omega_{0}-\omega_{s}\right) \mathrm{t}}+e^{-j\left(\omega_{0}+\omega_{S}\right) \mathrm{t}}\right) e^{-j \omega t} d t \\
& =\frac{A_{R}}{4} \cdot\left(2 \frac{\sin \left[\left(\omega_{0}-\omega\right)^{T / 2}\right]}{\omega_{0}-\omega}+\frac{\sin \left[\left(\omega_{0}-\omega_{s}-\omega\right)^{T} / 2\right]}{\omega_{0}-\omega_{s}-\omega}+\frac{\sin \left[\left(\omega_{0}+\omega_{s}-\omega\right)^{T} / 2\right]}{\omega_{0}+\omega_{s}-\omega} \ldots\right. \\
& \left.+2 \frac{\sin \left[\left(\omega_{0}+\omega\right)^{T / 2}\right]}{\omega_{0}+\omega}+\frac{\sin \left[\left(\omega_{0}-\omega_{s}+\omega\right)^{T} / 2\right]}{\omega_{0}-\omega_{s}+\omega}+\frac{\sin \left[\left(\omega_{0}+\omega_{s}+\omega\right)^{T} / 2\right]}{\omega_{0}+\omega_{s}+\omega}\right) \\
& +j \frac{A_{I}}{4} \cdot\left(2 \frac{\sin \left[\left(\omega_{0}-\omega\right)^{T} / 2\right]}{\omega_{0}-\omega}+\frac{\sin \left[\left(\omega_{0}-\omega_{s}-\omega\right)^{T} / 2\right]}{\omega_{0}-\omega_{s}-\omega}+\frac{\sin \left[\left(\omega_{0}+\omega_{s}-\omega\right)^{T} / 2\right]}{\omega_{0}+\omega_{s}-\omega} \ldots\right. \\
& \left.-2 \frac{\sin \left[\left(\omega_{0}+\omega\right)^{T / 2}\right]}{\omega_{0}+\omega}-\frac{\sin \left[\left(\omega_{0}-\omega_{s}+\omega\right)^{T} / 2\right]}{\omega_{0}-\omega_{s}+\omega}-\frac{\sin \left[\left(\omega_{0}+\omega_{s}+\omega\right)^{T} / 2\right]}{\omega_{0}+\omega_{s}+\omega}\right) .
\end{aligned}
$$

Therefore, $S_{H}\left(\omega_{0}, \omega\right)$ in general is:

$$
\Rightarrow S_{H}\left(\omega_{0}, \omega\right)=A_{R}\left[B_{A}\left(\omega_{0}, \omega\right)+B_{B}\left(\omega_{0}, \omega\right)\right]+j A_{I}\left[B_{A}\left(\omega_{0}, \omega\right)-B_{B}\left(\omega_{0}, \omega\right)\right]
$$

where

$$
\begin{aligned}
& B_{A}\left(\omega_{i}, \omega\right)=2 \frac{\sin \left[\left(\omega_{i}-\omega\right)^{T} / 2\right]}{\omega_{i}-\omega}+\frac{\sin \left[\left(\omega_{i}-\omega_{s}-\omega\right)^{T} / 2\right]}{\omega_{i}-\omega_{s}-\omega}+\frac{\sin \left[\left(\omega_{i}+\omega_{s}-\omega\right)^{T} / 2\right]}{\omega_{i}+\omega_{s}-\omega} \\
& B_{B}\left(\omega_{i}, \omega\right)=2 \frac{\sin \left[\left(\omega_{i}+\omega\right)^{T} / 2\right]}{\omega_{i}+\omega}+\frac{\sin \left[\left(\omega_{i}-\omega_{s}+\omega\right)^{T} / 2\right]}{\omega_{i}-\omega_{s}+\omega}+\frac{\sin \left[\left(\omega_{i}+\omega_{s}+\omega\right)^{T} / 2\right]}{\omega_{i}+\omega_{s}+\omega} .
\end{aligned}
$$

With this, the projection matrix can be calculated similar than in Eq. 4.14 on page 45:

$$
\bar{\Gamma}\left[f_{k}\right]=[\overbrace{\left[\begin{array}{c}
\left\{S_{H}\left(f_{1}, f_{k}\right)\right\}_{k=-T f_{S} / 2}^{T f_{S} / 2-1} \\
\vdots \\
\vdots
\end{array}\right]}^{\left\{S_{H}\left(f_{N}, f_{k}\right)\right\}_{k=-T f_{S} / 2}^{T f_{S} / 2-1}}] .
$$


After the selection of $N$ rows, $\Gamma$ can be used at the SSDM receiver using Eq. 4.15 on page 47 .

\subsubsection{Heterogeneous sub-carriers}

The methods for higher SE than OFDM shown in Section 3.2 on page 25 are restricted to homogeneous carriers meaning that $\triangle f=\alpha / T$. Meanwhile, in SSDM the frequency step factor $\triangle f_{i}$ can be arbitrary and heterogeneous from one subcarrier to another.

In essence, nothing has to change to generate either heterogeneous or homogeneous subcarriers as long as the receiver knows the modulating frequencies $f_{i}$. Other carrier parameters in the detector, such as $\Gamma, T, f_{S}$, require to be adjusted to match the transmitter's. Figure 4.5 shows an example of two SSDM systems both with 5 subcarriers modulating with QPSK. Figure 4.5a displays homogeneous carriers while $4.5 \mathrm{~b}$ heterogeneous.

In Figure 4.5, the subcarriers spectrum is marked with + . The black solid line marked with $\square$ responds to the composite spectrum of the SSDM carrier. The steams with a circle in the top represent the spectrum sampling that has been done in the receiver at the frequencies $f_{i}$. Despite the fact that all the subcarriers have the same magnitude, the SSDM carrier shows variations because the phases of the subcarriers are different. The magnitude is shown in arbitrary scale. In Figure 4.5a, the subcarriers have been distributed evenly with a frequency step of $\alpha=0.75$. Meanwhile in Figure $4.5 \mathrm{~b}$ on the following page, the frequency step is heterogeneous. For instance, the first two subcarriers are only one spectral sample apart with $\alpha_{1 \leftrightarrow 2}=0.25$. Also, the last carrier has been shifted one step to the right leading to a bigger carrier bandwidth. The normalized frequency step in this heterogeneous carrier averages $\alpha_{\text {avg }}=0.81$.

It is seen through experimentation that even subcarrier distributions have better BER performance than heterogeneous subcarriers. Additionally, other systems such as OFDM, SEFDM and OvFDM are all homogeneous. Therefore, for comparison purposes, the tests performed in this thesis are all done with uniform sinusoidal subcarriers although the nonuniform mode of SSDM has been proven successfully. 


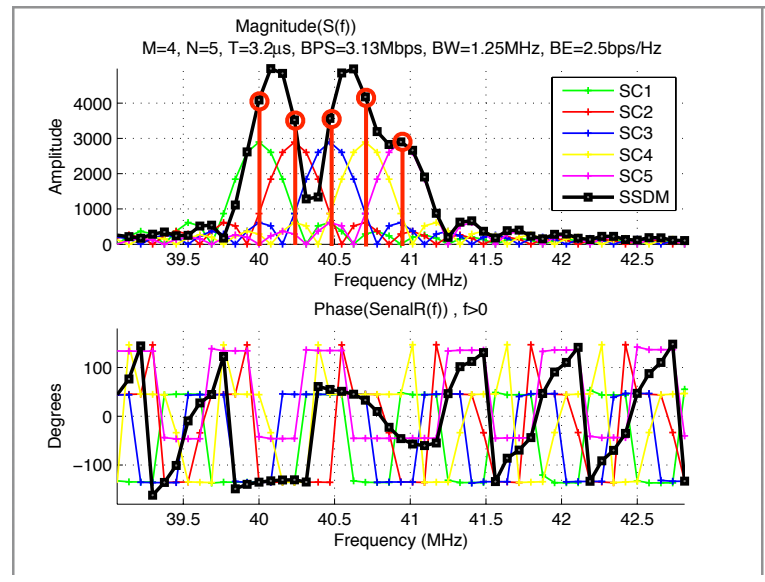

(a) Homogeneous carriers in SSDM

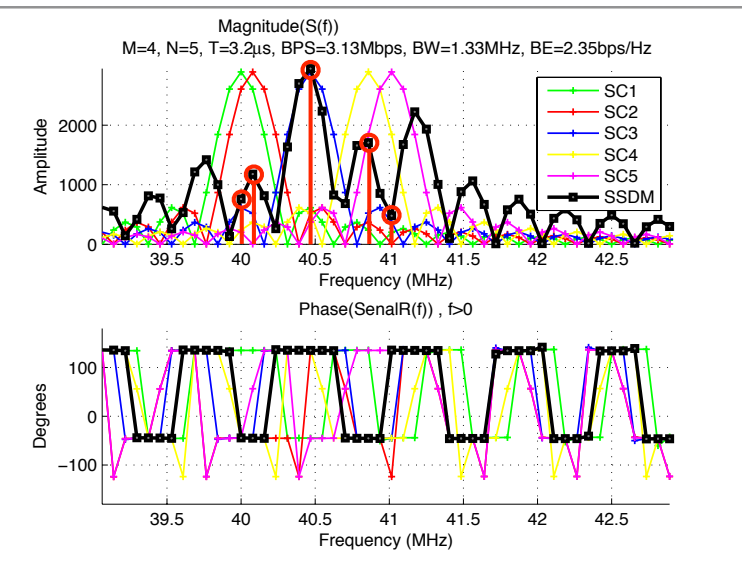

(b) Heterogeneous carriers in SSDM

Figure 4.5: Homogeneous and heterogeneous subcarriers 


\section{Chapter 5}

\section{Implementation}

Chapter 4 introduced the equations necessary for the SSDM transceiver. In this Chapter, those equations are used in the simplest form to produce a computer efficient design.

\subsection{Transmitter}

There are several ways to implement a transmitter for spectrally efficient systems. The most common is OFDM as seen in Section 3.1 as well as SE methods as shown in Section 3.2 on page 25. These systems are designed to generate heterogeneous subcarrier signals with the same basic signal shape for each subcarrier. SSDM, on the other hand, is able to use subcarriers at arbitrary frequencies and arbitrary signal shape. This section shows a possible implementation of the SSDM transmitter.

The transmitter performs a weighted addition of the subcarrier signals to generate the transmittable signal $s(t)$. The weighting factors for every signal are $A_{R i}$ for the signals based on cosine and $-A_{I i}$ for the signals based on sine according to Table 4.2. Figure 5.1 shows an example of the architecture of this transmitter. As a measure of hardware complexity, the time domain transmitter requires $2 N T / T_{S}$ real floating point multiplications and $T / T_{S}$ additions.

A time domain transmitter is based on the process shown in Table 4.2. In this design, a pool of $2 N$ real base signals are sampled and stored in memory. $N$ signals are cosine functions $\cos \left(2 \pi f_{i} t\right)$ and $N$ signals are sine functions $\sin \left(2 \pi f_{i} t\right)$ where $f_{i}$ is the frequency 


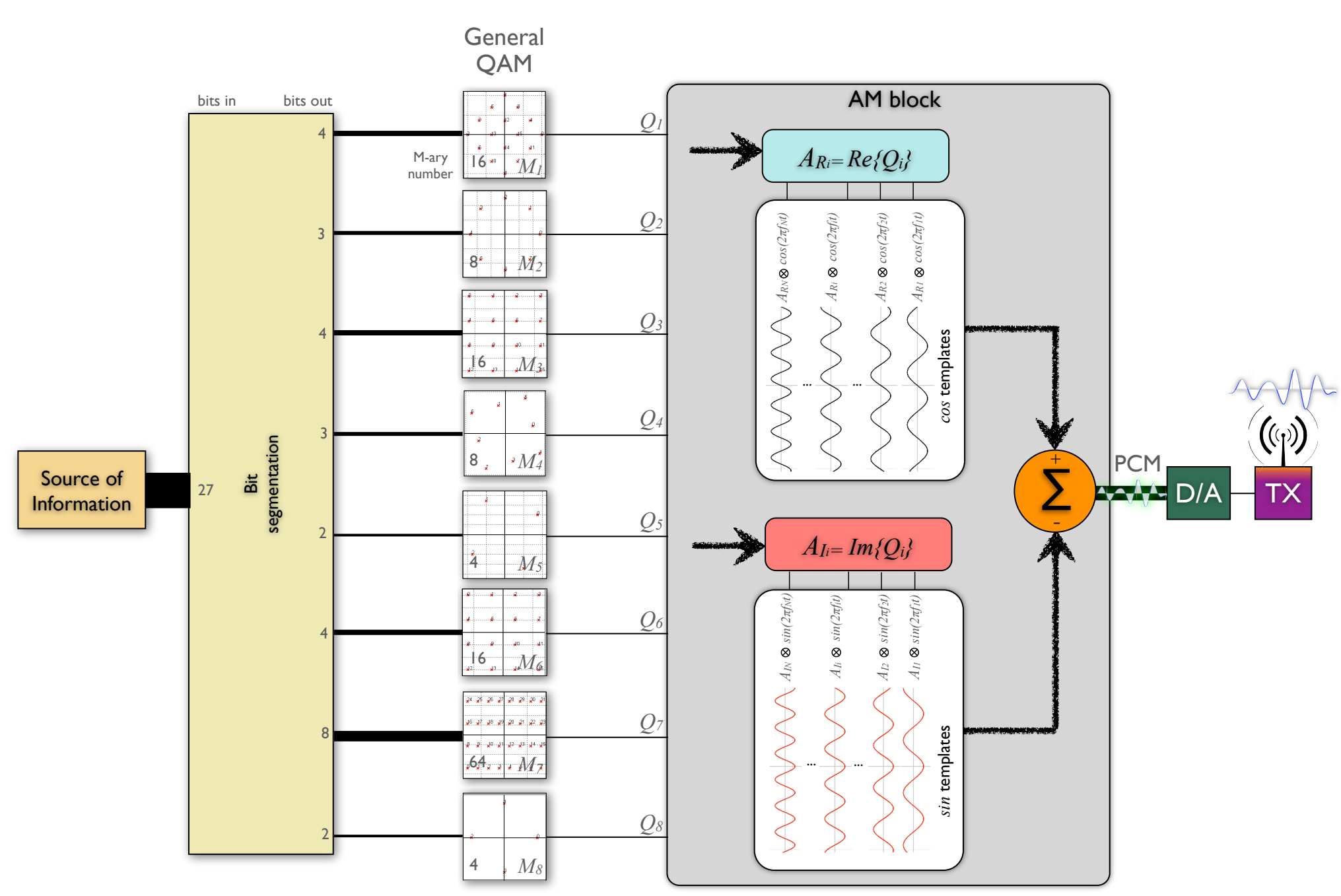

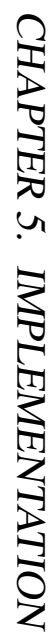

Figure 5.1: SSDM transmitter example 
of the subcarrier $i$. The number of samples for these signals is equal to the symbol length $T / T_{S}$. These signals can be longer by preference to generate a CP. The receiver can remove the $\mathrm{CP}$ as explained in Section 3.1 and Figure 3.8 on page 24 to prevent the ISI that results from multipath propagation.

\subsection{Receiver}

The SSDM receiver consists of an optional down-converter, an ADC, a tracking block that removes the CP, a serial to parallel converter, a fast Fourier transform (FFT) block, a detection stage, a classifier and a bit assembler.

The complex form of Eq. 4.12 is

$$
\left[\begin{array}{c}
\vdots \\
S\left(f_{k+1}\right) \\
S\left(f_{k}\right) \\
S\left(f_{k-1}\right) \\
\vdots
\end{array}\right]=\left[\begin{array}{cccc}
\vdots & \vdots & \cdots & \vdots \\
S_{1}\left(f_{k+1}\right) & S_{2}\left(f_{k+1}\right) & & S_{N}\left(f_{k+1}\right) \\
S_{1}\left(f_{k}\right) & S_{2}\left(f_{k}\right) & \ddots & S_{N}\left(f_{k}\right) \\
S_{1}\left(f_{k-1}\right) & S_{2}\left(f_{k-1}\right) & & S_{N}\left(f_{k-1}\right) \\
\vdots & \vdots & \cdots & \vdots
\end{array}\right] \cdot\left[\begin{array}{c}
Q_{1} \\
Q_{2} \\
\vdots \\
Q_{N}
\end{array}\right]
$$

which is equivalent to Eq. 4.13. To make this equation invertible, only $N$ rows are used. By doing this, the modulating constants $Q_{i}$ can be estimated at the receiver as shown in Eq. 4.15 .

An approach to calculate $\Gamma^{-1}$ is using Eq. 4.14. For this, $\Gamma$ needs to be square. Therefore, $\Gamma^{-1}$ is calculated using only the $N$ rows that contain the $f_{k}$ of interest. The frequencies selected are the ones that match the $N$ samples at the output of the FFT block. Let the $N$ samples be $f_{a}, f_{b}$ to $f_{Z}$ respectively. Meanwhile, $f_{1}$ to $f_{N}$ are the frequencies of the SSDM subcarriers; in other words, constants. Therefore, $\Gamma^{-1}$ can be obtained from

$$
\bar{\Gamma}\left[f_{k}\right]=[\overbrace{\left[\begin{array}{c}
\left\{R_{1}\left(f_{k}\right)\right\}_{k=\left\{k_{a}, k_{b}, \ldots, k_{m}\right\}} \\
\vdots \\
(1+j) \frac{\sin \left[\pi\left(f_{1}-f_{k}\right) T\right]}{2 \pi\left(f_{1}-f_{k}\right)} \\
\vdots
\end{array}\right]}^{\left\{R_{2}\left(f_{k}\right)\right\}_{k=\left\{k_{a}, k_{b}, \ldots, k_{m}\right\}}} \begin{array}{c}
\vdots \\
\left.\vdots \begin{array}{c}
(1+j) \frac{\sin \left[\pi\left(f_{2}-f_{k}\right) T\right]}{2 \pi\left(f_{2}-f_{k}\right)} \\
\vdots
\end{array}\right]
\end{array}]
$$


which is equal to

$$
\bar{\Gamma}\left[f_{k}\right]=\left[\begin{array}{cccc}
(1+j) \frac{\sin \left[\pi\left(f_{1}-f_{a}\right) T\right]}{2 \pi\left(f_{1}-f_{a}\right)} & (1+j) \frac{\sin \left[\pi\left(f_{2}-f_{a}\right) T\right]}{2 \pi\left(f_{2}-f_{a}\right)} & \ldots & \vdots \\
(1+j) \frac{\sin \left[\pi\left(f_{1}-f_{b}\right) T\right]}{2 \pi\left(f_{1}-f_{b}\right)} & (1+j) \frac{\sin \left[\pi\left(f_{2}-f_{b}\right) T\right]}{2 \pi\left(f_{2}-f_{b}\right)} & \ddots & \vdots \\
\vdots & \vdots & \cdots & (1+j) \frac{\sin \left[\pi\left(f_{N}-f_{Z}\right) T\right]}{2 \pi\left(f_{N}-f_{Z}\right)}
\end{array}\right]
$$

The estimated symbols at the receiver are then

$$
\left[\begin{array}{c}
Q_{a}^{\prime} \\
Q_{b}^{\prime} \\
\vdots \\
Q_{m}^{\prime}
\end{array}\right]=\bar{\Gamma}^{-\mathbf{1}}\left[f_{k}\right] \cdot\left[\begin{array}{c}
S^{\prime}\left(f_{a}\right) \\
S^{\prime}\left(f_{b}\right) \\
\vdots \\
S^{\prime}\left(f_{m}\right)
\end{array}\right]
$$

where $Q_{i}^{\prime}$ is the estimated value of $Q_{i}$ and $S^{\prime}(f)$ corresponds to the spectrum of the received signal taken from $N$ outputs of the FFT block. The $N$ samples selected correspond to the frequencies of interest. This frequencies are preferably the center frequencies of the subcarriers. Otherwise, the frequencies available at the output of the FFT block are $\left\{-f_{S} / 2,-f_{S} / 2+1 / T, \ldots, f_{S} / 2-1 / T\right\}$ in correspondence to their respective $k$ 's which are $\left\{-T f_{S} / 2,-T f_{S} / 2+1, \ldots, T f_{S} / 2-1\right\}$ through the relationship $f_{k}=k / T$. High $k$ 's should be selected to satisfy the assumption made in Eq. 4.10.

The FFT block has $T / \alpha T_{S}$ inputs and same amount of outputs. The selection of the desired samples is not necessarily related to the ones that match every subcarrier $f_{i}$. The use of FFT blocks is possible by adjusting the size of the symbol so the amount of inputs to the block is $2^{r}$ where $r$ is an integer. This adjustment can be done by zero padding the received signal. Otherwise, a DFT block can be used. Besides that, the required sampling frequency is inversely proportional to the space between subcarriers and directly proportional to the symbol length. This is $f_{S} \propto \alpha^{-1}$ and $f_{S} \propto T$. Similarly, the number of complex multiplications depends on $N^{2}$ and the number of complex additions on $(N-1) N$.

The SSDM receiver is shown in Figure 5.2. In this receiver, the signal is converted into digital form with an ADC. A tracking block detects the beginning of the signal period and removes the CP. A serial to parallel converter delivers the symbol to an FFT block. 
The FFT block provides $N$ samples to the detection stage. The estimated symbols are then classified and placed in serial form to the information end. 


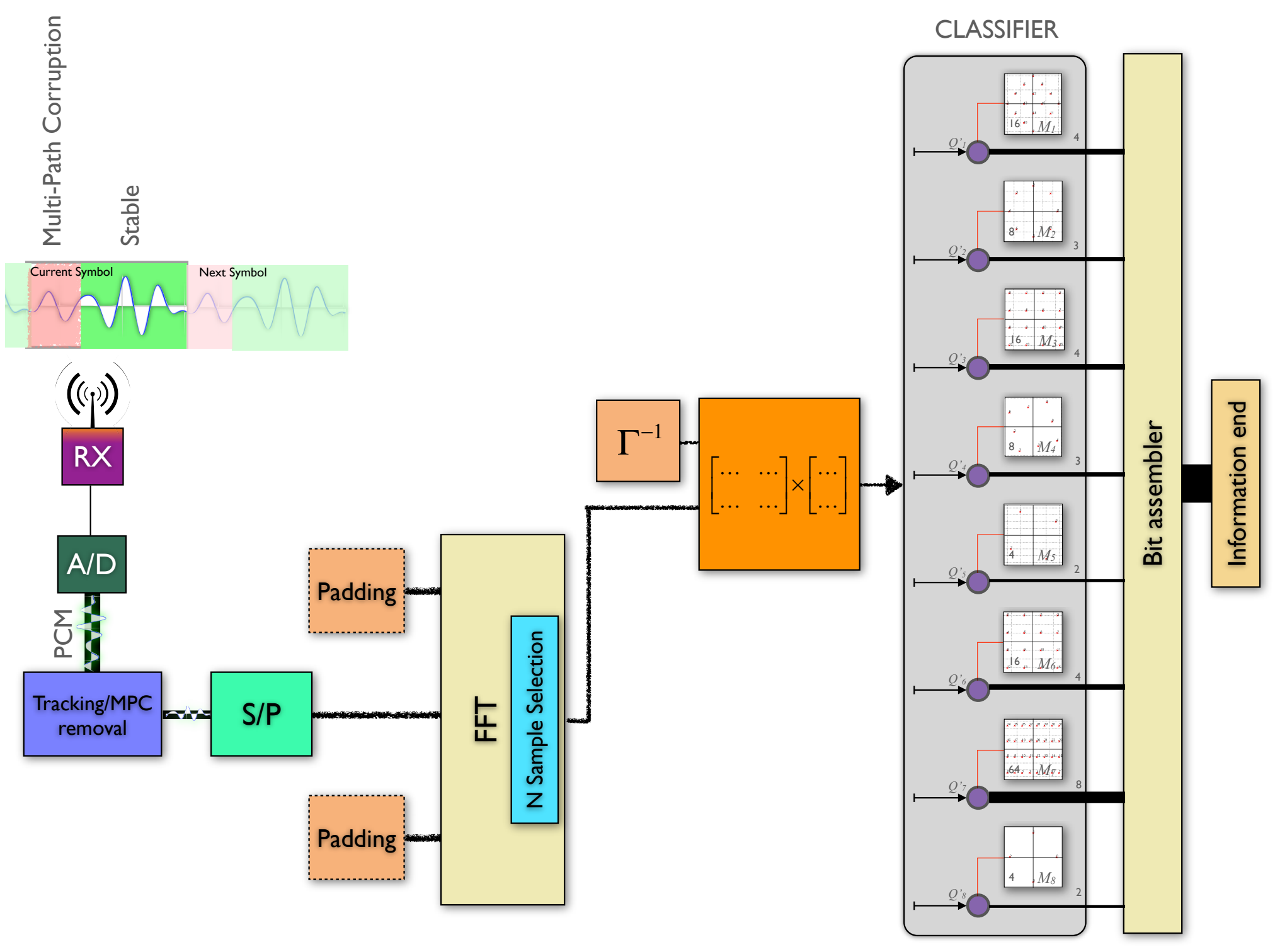

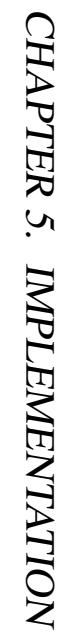

Figure 5.2: SSDM receiver 


\section{Chapter 6}

\section{Experimental Model Set-Up}

\subsection{Monte-Carlo analysis}

Monte-Carlo analysis is used to test the SSDM communications system. The Monte-Carlo analysis consists in stressing the system with a random information source until the BER converges. During the tests system parameters such as $T, f_{s}, f_{i}, M_{i}$ and SNR are fixed. One of the parameters is changed before the test is repeated. The analysis is performed for both the transmitter and the receiver. The block diagram of the model is shown in Figure 6.1. If the system parameters $M_{i}, T, f_{S}$, and $\Gamma$ are fixed, the blocks in gray shown in Figure 6.1 can be pre-computed off-line. Meanwhile, the blocks in white perform real time operations.

The transmitter consists of a database of symbols formed by groups of $N$ random integers that go from 0 to $2^{M_{i}}-1$. These integers are individually mapped on their respective constellations $Q_{i}$. The output of the QAM mapper is $N$ complex numbers. Normalized waveforms have been stored in the function generator block. In this case, these waveforms are sine and cosine as in Table 4.2. The real and imaginary components are used to scale each of the waveforms stored individually. These weighted functions are mixed together by addition to generate the SSDM signal. A noise generator incorporates AWGN to the symbol.

The receiver, consists of an FFT block that calculates the spectrum of the signal. $N$ of these values are multiplied by $\Gamma^{-1}$ with a Vector-Matrix multiplication block. The output consists of the estimated symbols $Q_{i}^{\prime}$. These symbols are classified and stored on 


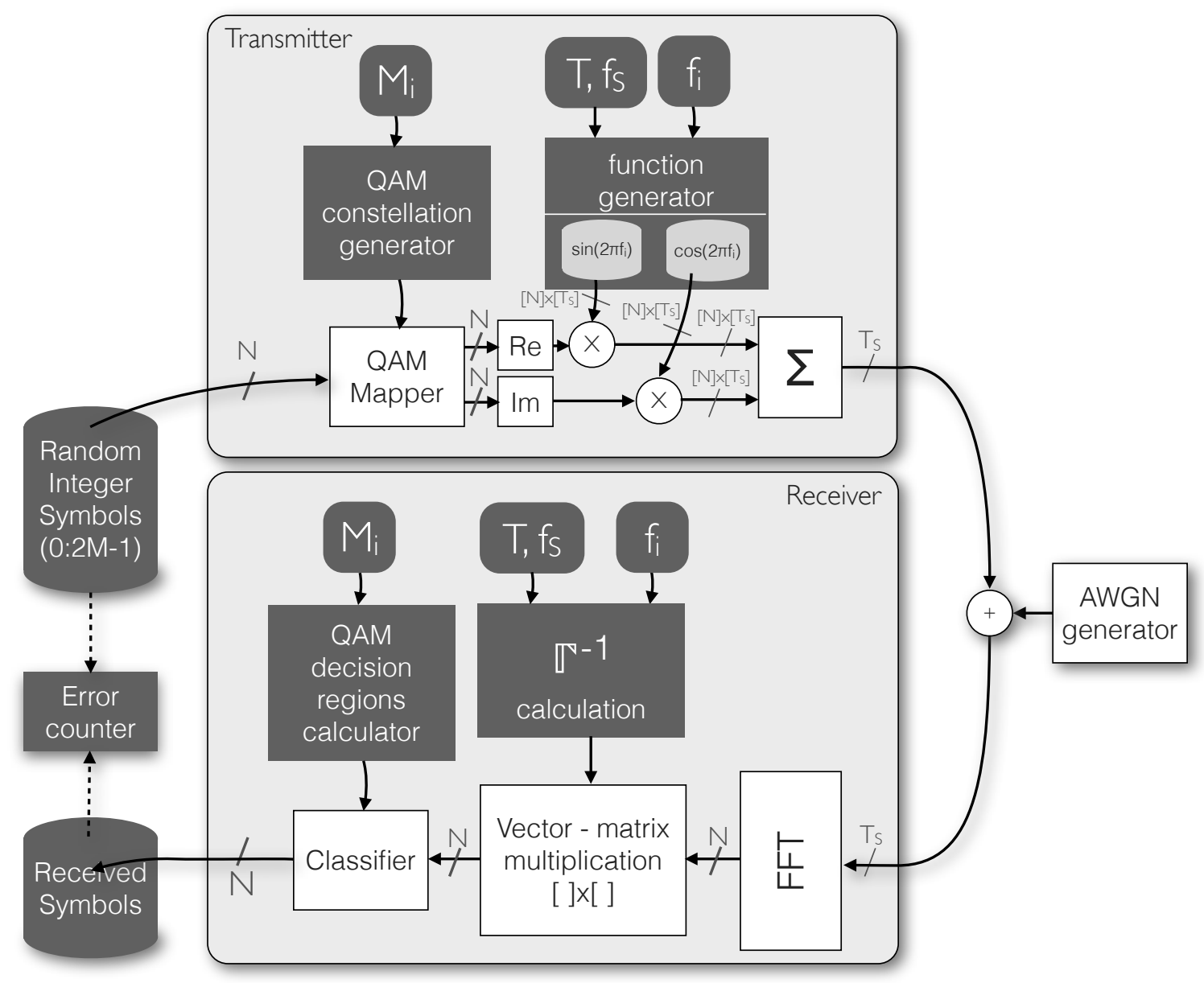

Figure 6.1: Monte-Carlo analysis on the SSDM system model

a database that resembles the information end. The test model was implemented using Matlab $^{\mathrm{TM}} \mathrm{r} 2011 \mathrm{~b}$.

\subsection{Algorithm}

The test is run until all the symbols of the random source are depleted. The received bits are compared one by one with the original bits by an error counter block. The error count is saved for comparison. After that is done, one parameter - for instance, the power of the noise generator - is changed and the test is repeated systematically. The pseudocode of the process loop is represented in Algorithm 6.1. 


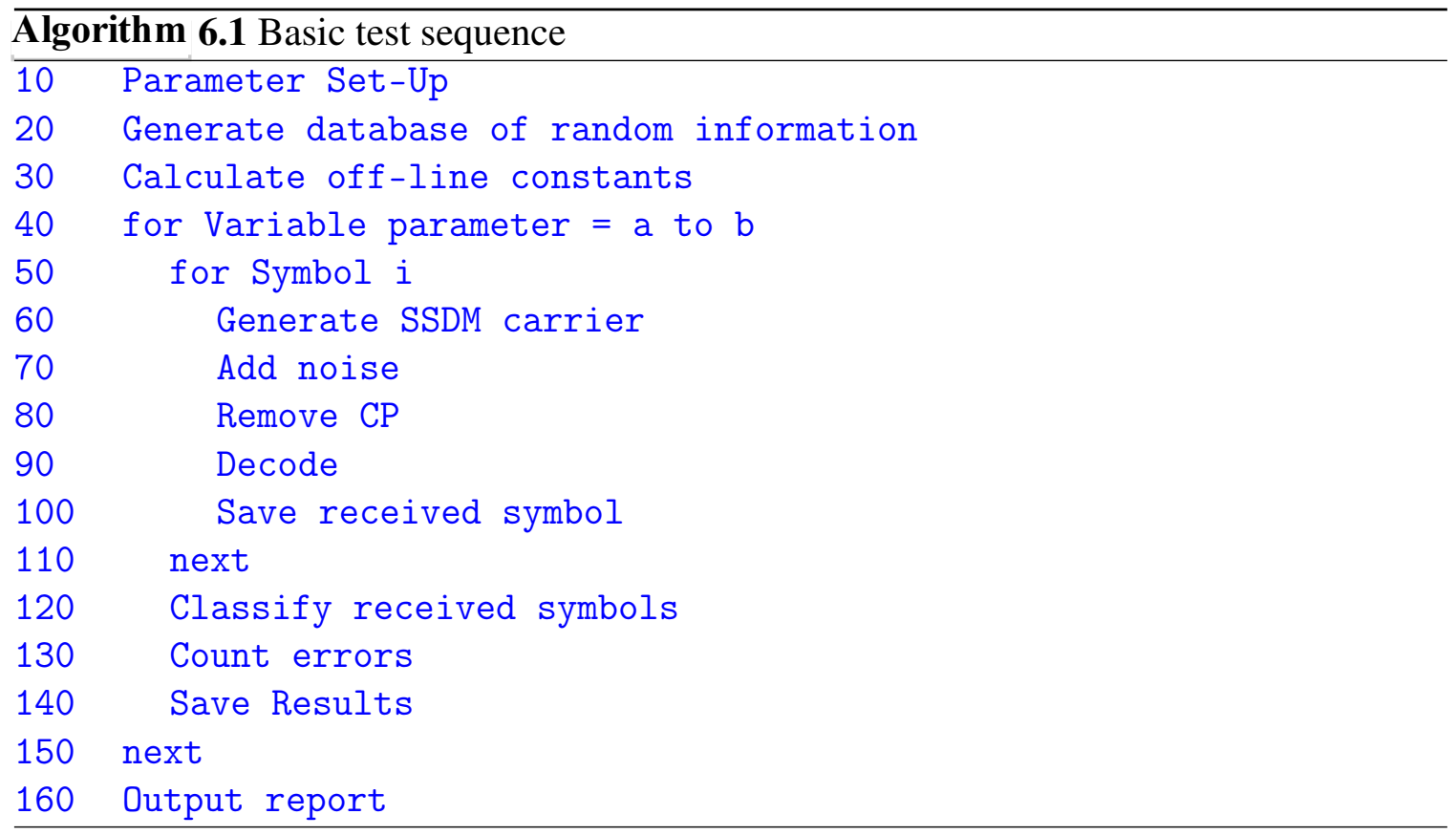

\subsection{Tests}

In these tests the main parameters have been set to $T=3.2 \mu \mathrm{s}, f_{1}=\frac{128}{T}=40 \mathrm{MHz}, f_{S}=$ $32 f_{1}=1.28 \mathrm{GHz}, M_{i}=M=4$ and $\alpha=0.8$. Some of the tests performed are:

\section{BER performance vs SNR}

The test is repeated by varying the SNR. The BER measure is taken straight from the error counter block.

\section{Effective spectral efficiency (ESE) vs SNR}

The test is repeated by varying the subcarrier separation factor $\alpha$. The SE is calculated based on the used bandwidth and the carrier speed. Just as, the bandwidth is computed based on the dimension of the carrier $N$, the bits of the constellations $k$, the carrier separation $\alpha$ and the symbol period $T$.

$$
\begin{aligned}
\mathrm{BW} & =\frac{(N-1) \alpha+2}{T} \\
\mathrm{ESE} & \triangleq \frac{k \cdot N(1-\mathrm{BER})}{\mathrm{BW} \cdot T}
\end{aligned}
$$


In this work, the effective spectral efficiency (ESE) is defined as the number of nonerror bits sent per unit of spectrum. This indicator provides a more reliable and complete measure of performance of communication systems as shown on page 63 .

\section{Noise scale}

To compare the SSDM system with others, the signal to noise ratio (SNR) scale has been converted to bit energy over noise ratio $\mathrm{E}_{b} / \mathrm{N}_{0}$. The relationship between these noise ratios depends on the number of possible electric levels, or order of modulation, and is given by

$$
\left(\mathrm{E}_{\mathrm{b}} / \mathrm{N}_{0}\right)_{\mathrm{dB}}=(\mathrm{SNR})_{\mathrm{dB}}+10 \log (k),
$$

where $k=\log _{2} M$ and $M$ is the order of the QAM constellation. 


\section{Chapter 7}

\section{Results}

\subsection{Comparison with OFDM}

A promising feature of SSDM, and other SE techniques, is that it is backwards compatible with OFDM. Indeed, OFDM is a special case of SSDM. This makes the SSDM system easier to deploy in the industry. When $\alpha=1$, the SSDM carrier behaves equal than the OFDM carrier. Moreover, when the subcarriers are orthogonal $\triangle f=1 / T$, the Vector-Matrix multiplication block can be omitted from the receiver without changing the results. In that case, the output of the FFT block is not multiplied by $\Gamma^{-1}$ as in Figure 5.2 on page 57 but is connected classifier directly as in Figure 3.7 on page 24 . At orthogonallity the matrix $\Gamma$ becomes identity ${ }^{1}$ because the subcarriers are spectrally independent. Therefore, the SSDM receiver with $\alpha=1$ performs exactly like OFDM even with high dimensionality carriers. The next section compares the performance of SSDM including OFDM.

\subsection{Performance}

Figure 7.1 shows the relationship between BER performance and the bit-energy to noise ratio $\mathrm{E}_{b} / \mathrm{N}_{0}$ for a certain normalized frequency step $\alpha$. In Figure 7.1, $N=12$ and $\alpha$ decreases from 1 to 0.59 . The number of carriers used for this figure is $N=12$ with QPSK $M=4$ and

\footnotetext{
${ }^{1}$ There is a scaling factor. Additionally, it is assumed that the selected rows of the projection matrix correspond to the subcarrier frequencies $f_{i}$.
} 
the symbol period $T=3.2 \mu \mathrm{s}$. The rate of the information is $B P S=N \cdot \log _{2} M / T=7.5 \mathrm{Mbps}$. The frequency of the first carrier is $f_{0}=2^{7} / T=40 \mathrm{MHz}$ which takes care of the assumption made in Eq. 4.6 on page 41. The sampling frequency is set to $f_{S}=2^{5} \cdot f_{0}=1.28 \mathrm{GHz}$ which reduces the impact of sampling aliasing ${ }^{2}$ [34]. These relationships guarantee that the input to the Fourier transform block would be a power of 2 which allows the indistinct use of either the fast Fourier transform ${ }^{3}$ (FFT) or discrete Fourier transform (DFT).

Figure 7.1 shows that, for a fixed $\mathrm{E}_{b} / \mathrm{N}_{0}$, as the normalized subcarrier step decreases $\alpha$, the BER grows. Similarly, the $\mathrm{E}_{b} / \mathrm{N}_{0}$ required to target certain BER is greater when $\alpha$ decreases. Additionally, the dashed line with $\alpha=0.59$ shows that, depending on the system parameters, there is a limit on the reduction of BER that can be achieved when the bitenergy to noise ratio $\mathrm{E}_{b} / \mathrm{N}_{0}$ increases. This indicates that there is an asymptotic limit in the BER for every level of carrier compression in SSDM. The asymptotes in the other curves are located below BER $=10^{-4}$ and are not seen in the figure. Although the asymptotes causes errors, communication is still possible since the probability of a successful bit is $(1-\mathrm{BER})$.

Figure 7.1 indicates that, for a certain ratio of signal strength, the BER is inversely proportional to the frequency step BER $\propto \alpha^{-1}$. On the other hand, from Figure 3.10b on page 28 , the utilization of the spectrum is directly linked to the frequency step BW $\propto \alpha$. Although this indicates that there is a relationship between the bandwidth used and the error rate $\mathrm{BW} \propto \mathrm{BER}^{-1}$, the graphics still tell little about the actual throughput of the carriers. In this sense, the effective spectral efficiency (ESE) is defined in Eq. 6.1 on page 60 as a measure of performance based on the bit rate, the error rate and the occupied bandwidth.

The relationship between subcarrier step and ESE is shown in Figure 7.2. In addition, Figure 7.2 includes the relative spectral gain compared with OFDM in percentile at the top of each curve. Figure 7.2a shows that for a signals with $\alpha=0.5, \mathrm{E}_{b} / \mathrm{N}_{0}=10 \mathrm{~dB}$ and small carrier size $N=5$ there is an increase of up to $33 \%$ in throughput in comparison with OFDM. Figure 7.2a also shows that the ESE depends directly on the signal strength. Similarly, as $\mathrm{E}_{b} / \mathrm{N}_{0}$ increases, it is convenient to switch to a lower carrier step $\alpha$.

\footnotetext{
${ }^{2}$ A sampled signal would produce spectral copies at the sampling frequency. Since the spectrum of an RF pulse is infinitely wide, the rule of sampling two times faster than Nyquist can not be applied. Therefore, a high sampling frequency reduces the effect of aliasing.

${ }^{3}$ The FFT performs faster in Matlab ${ }^{\mathrm{TM}}$
} 


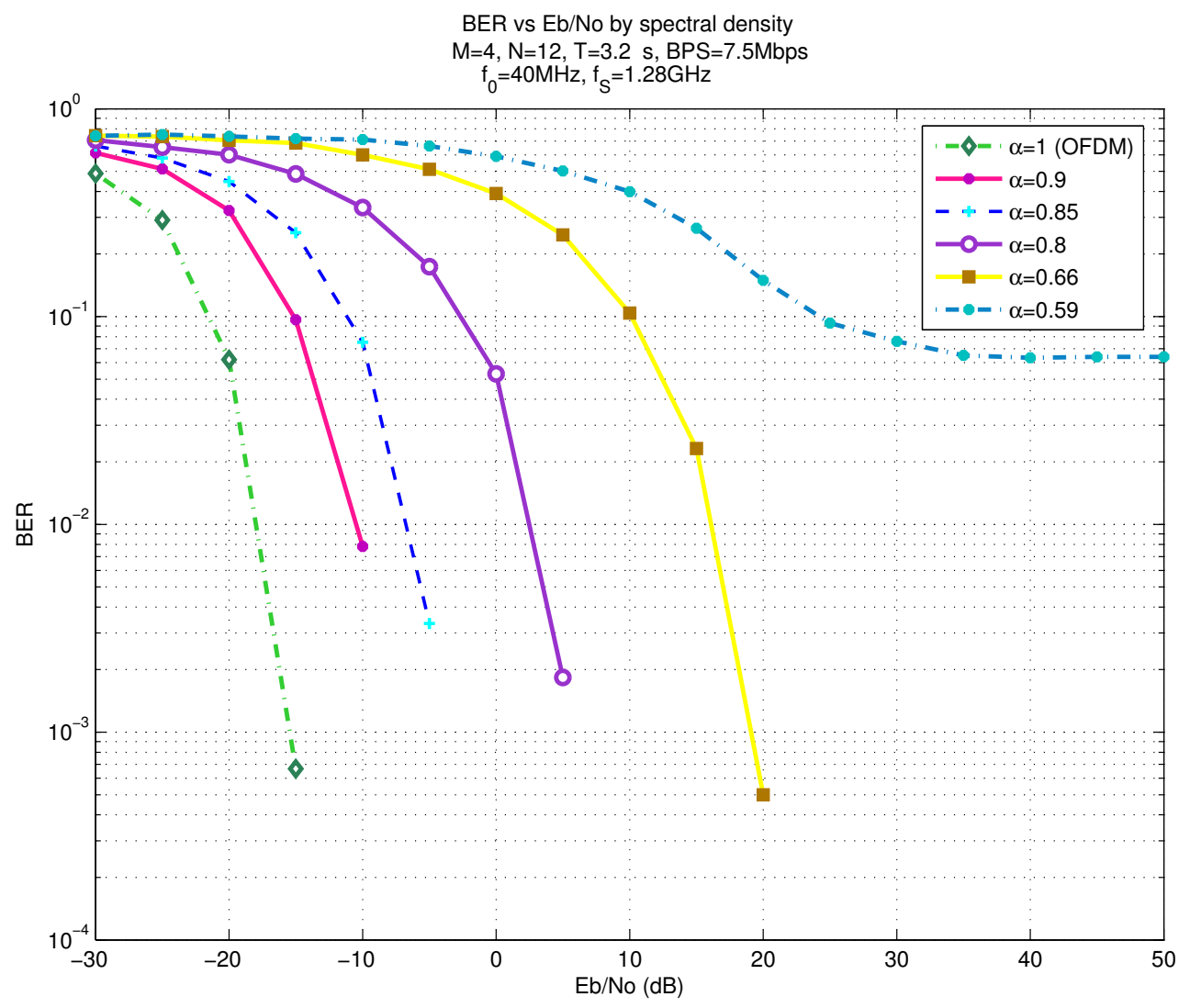

Figure 7.1: BER vs $\mathrm{E}_{b} / \mathrm{N}_{0}$ vs $\alpha$

Figure 7.2b shows a strange behavior at $\alpha=0.125$. On a carrier with high dimensionality $N=20$, the ESE increases as $\alpha$ approaches to zero. By looking at the curve with $\mathrm{E}_{b} / \mathrm{N}_{0}=5 \mathrm{~dB}$, which is above the one with $\mathrm{E}_{b} / \mathrm{N}_{0}=15 \mathrm{~dB}$, that behavior seems to be unrelated to the signal strength. Figure 7.3 helps explain this effect. The actual percentile gain over OFDM in Figure $7.2 \mathrm{~b}$ is $12 \%$ at $\alpha=0.875$.

In Figure 7.3, it is seen that carriers with certain constellation order $M$ have a defined tendency. For instance, carriers with $M=4$ approximate to a $B E R=0.75$ as the carrier dimension grows. This tendency is due to basic probability. The chances of getting the right symbol out of a source of 4 random symbols is $1 / 4$. Therefore, what the tendency really means is that, as $\alpha$ tends to zero, the received information is not related to the source anymore. The information is actually randomized by the system. This type of behavior 


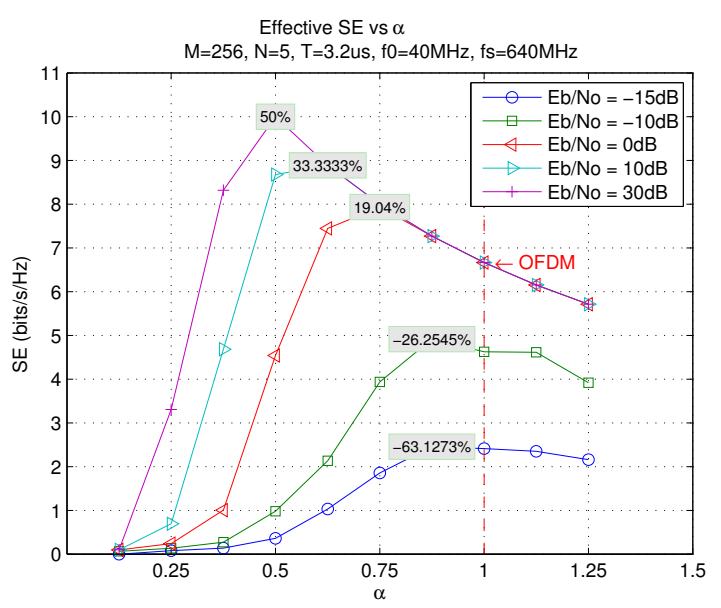

(a) $\mathrm{ESE}$ for $N=5, M=256$

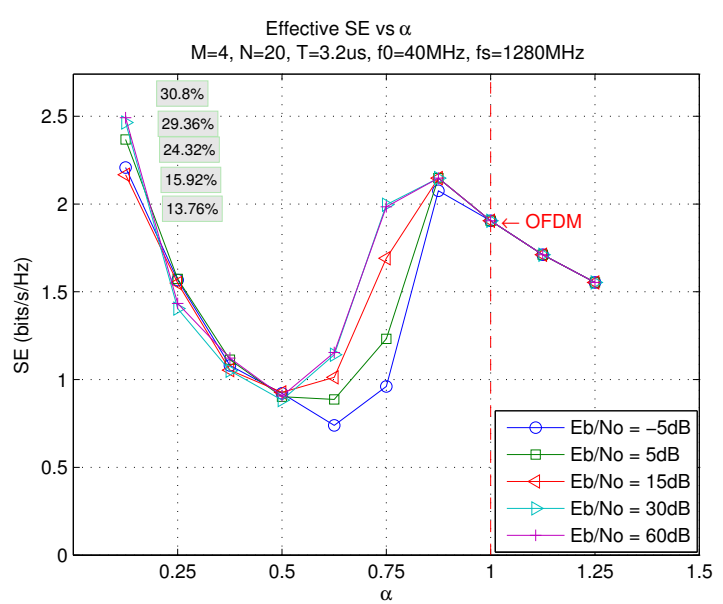

(b) $\mathrm{ESE}$ for $N=20, M=4$

Figure 7.2: Effective SE and percentile throughput gain with respect to OFDM

can not be fixed with convolutional codes because the decoded symbols are random anyway. By induction, $\lim _{\{N \rightarrow \infty \text { or } \alpha \rightarrow 0\}} \mathrm{BER}=1 / M$ which means that the system has become a random source and that the information is not recovered. Therefore, the peak at Figure $7.2 \mathrm{~b}$ is virtual, there is actually no communication. The curve responds to the probability of receiving the symbol the observer expects out of a random source of symbols which in QPSK is $25 \%$.

Figures 7.4a and 7.4b summarize the percentile gain over OFDM depending on the carrier size for signals with $E_{b} / N_{0}=0 \mathrm{~dB}$ and $E_{b} / N_{0}=10 \mathrm{~dB}$ respectively. The highest throughput gain occurs with smaller carriers in both number of subcarriers $N$ and constellation points M.

\subsection{Comparison with other methods}

The SSDM system can be compared with other SE techniques such as OFDM, SEFDM and OvFDM based on BER performance. Figure 7.5 shows the BER of SSDM compared with methods shown in other publications. Non of these simulations consider quantization noise due to $\mathrm{AD}$ converters. 


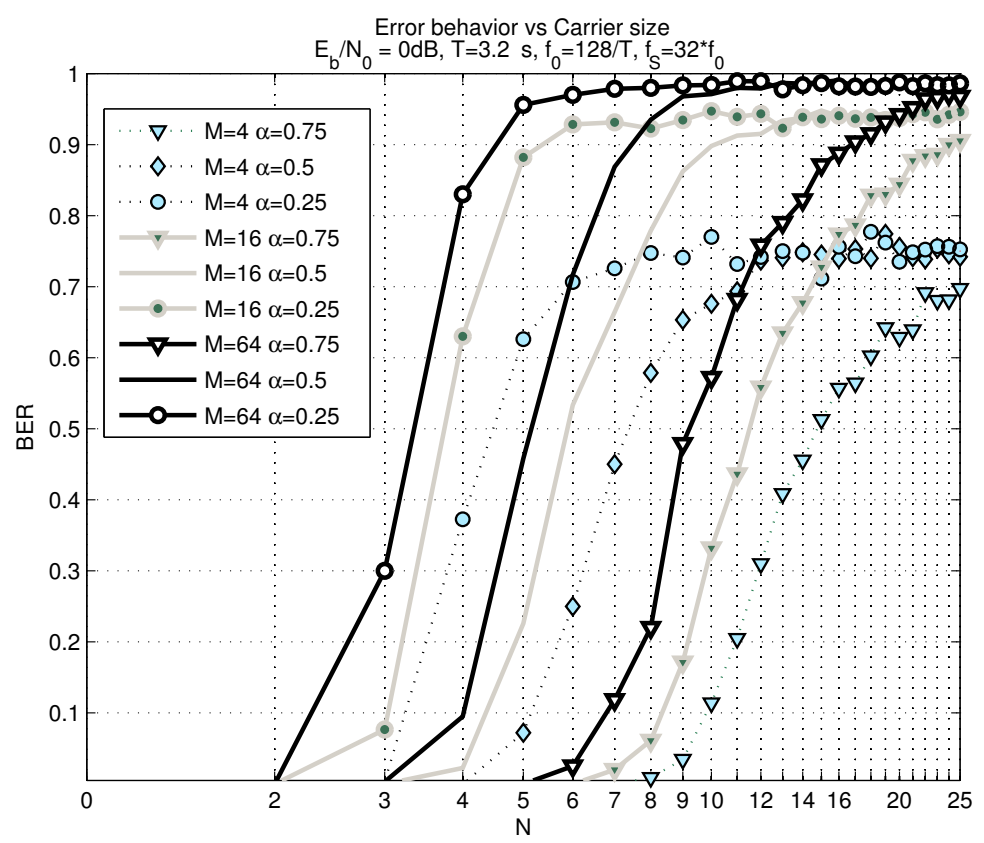

Figure 7.3: BER tendency by carrier dimension

The SSDM simulations in Figure 7.5 were made with $T=3.2 \mu \mathrm{s}, f_{0}=\frac{128}{T}=40 \mathrm{MHz}$, $f_{S}=32 f_{0}=1.28 \mathrm{GHz}$, QPSK $M_{i}=M=4$ and $\alpha=0.8$ unless noted. The total padding at the receiver is $3 T / T_{S}$ to complete $4 T / T_{S}$ samples at the input of the FFT block. In the legends on Figure 7.5, the numbers represent the dimension of the carrier. The meaning of these legends is summarized in Table 7.1.

It is observed that for high dimensionality carriers, the SSDM detector is no better than others. As seen in Figure 7.5, SSDM is unable to decode a carrier with $N=32$. Meanwhile,

\begin{tabular}{|c|c|}
\hline Label & Curve \\
\hline \hline$x$-SSDM & SSDM, $N=x$ \\
\hline 20-SSDM $\alpha=7 / 8 k=8$ & SSDM with 256-QAM, $M=2^{k}=256, N=x, \alpha=0.875$. Padding completes $8 T / T_{S}$ samples \\
\hline 32-FSD $\rho=2$ & Fixed sphere decoder with FFT block length $\rho N / \alpha[20], N=32$ \\
\hline 12-FSD-TSVD & Fixed sphere decoder with truncated singular value decomposition $[21], N=12$ \\
\hline 12-Precoded & Precoded detector based on ML and ZF [19], $N=12$ \\
\hline 32-RSD & Real valued fixed complexity SD for MIMO [43], $N=32$ \\
\hline 4-MMSE-ML & Combination of maximum likelihood with minimum mean square error [23], $N=4$ \\
\hline 56-OFDM 802.11a & OFDM with 23 carriers with $\alpha=1$, a gap of $\alpha=2$ and another 23 carriers with $\alpha=1, N=56$ \\
\hline
\end{tabular}

Table 7.1: Captions in Figure 7.5. 


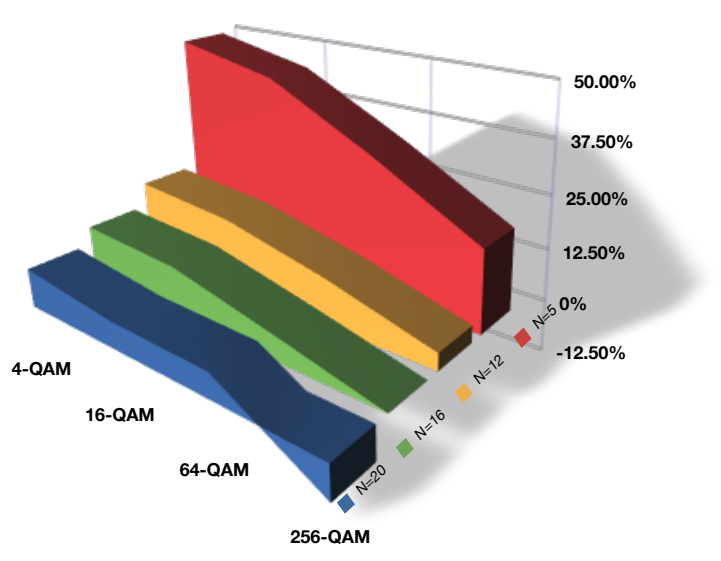

(a) $E_{b} / N_{0}=0 \mathrm{~dB}$

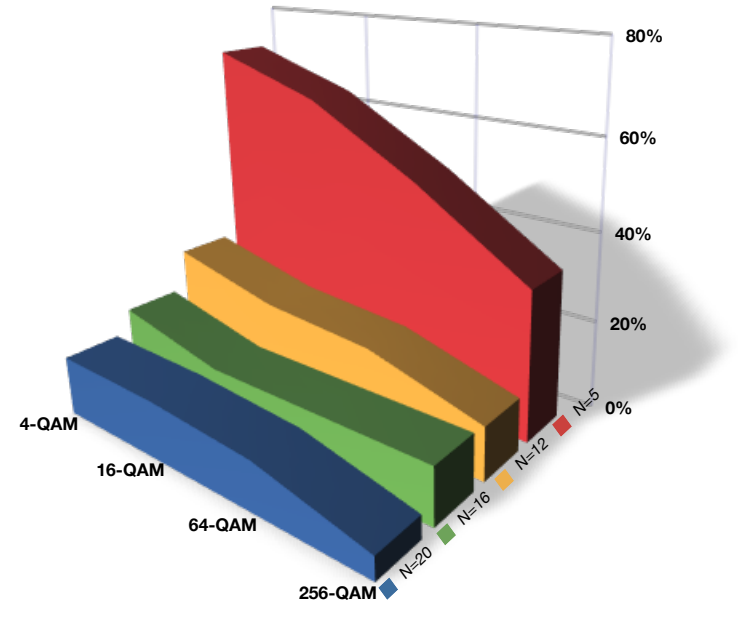

(b) $E_{b} / N_{0}=10 \mathrm{~dB}$

Figure 7.4: Percentile throughput gain of SSDM over OFDM vs carrier dimension

the real sphere decoder (RSD) decodes the signal properly as seen in the side of the graph with higher $\mathrm{E}_{b} / \mathrm{N}_{0}$. The dramatic difference in performance between other detectors and the RSD detector, is because the RSD is an algorithm for MIMO systems [43]. It has been only included to display the great difference of performance between SISO and MIMO systems.

When the dimensionality decreases to $N \leq 16$ the performance of SSDM is notable. The curve with marked with a black $\triangleleft$ corresponds to a carrier of size 16 . In this case, the SSDM performs even better than the FSD-TSVD detector with a carrier of lower dimensionality $N=12$. Additionally, the response of SSDM is ever more notorious when the dimension of the carrier is low. However, the performance is not higher than OFDM. Finally, it is seen that a communications system in SSDM would require higher SNR than OFDM to achieve a target BER. Equivalently, it would result in higher BER for a fixed SNR.

By sampling the incoming signal at high rates, the noise effects are diminished with the FFT block because it counts with more samples to rely on. Nevertheless, the disadvantage is that the receiver requires longer FFT blocks. This improvement at higher sampling rate is also found in recent publications [8]. The performance of the SSDM system drops as the sampling frequencies decrease as shown in Figure 7.6. This is due to the collapse of the 


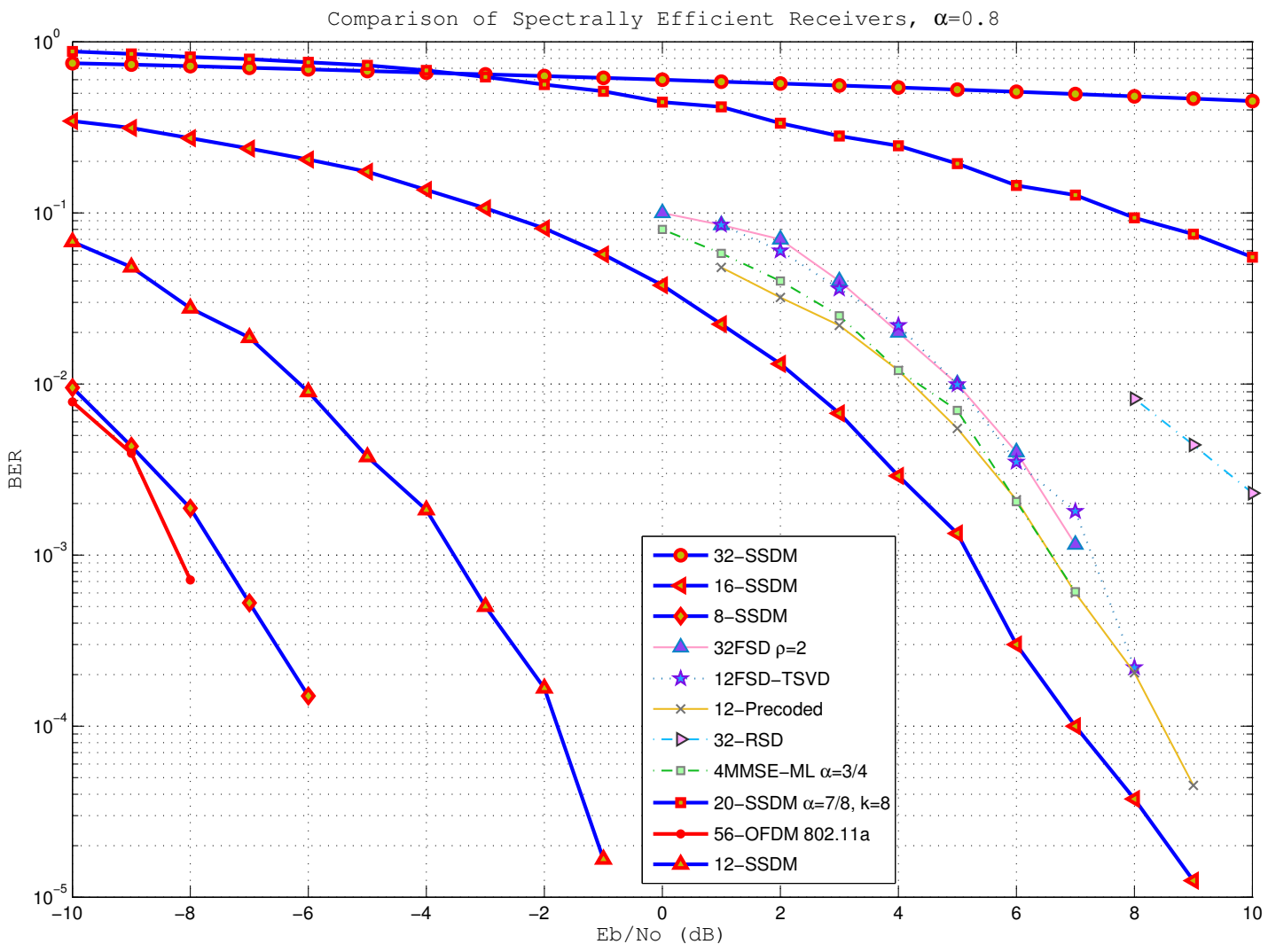

Figure 7.5: Performance comparison with other receivers with Q-PSK

sampling theorem. As $f_{S}$ approaches to $f_{i}$, spectral copies centered at $f_{S}-f_{i}$ interfere with the spectrum of the subcarriers at $f_{i}$. The reduced performance with lower sampling rates is shown in Figure 7.6. Therefore, an extension to Eq. 4.6 on page 41 is $f_{s}-f_{i} \gg f_{i}$ which is equivalent to $f \gg 2 f_{i}$. In sum, a theoretical constraint of the SSDM system is that the sampling frequency needs to be considerable higher than the Nyquist limit. Nevertheless, band pass filters at the receiver radio would help reduce this need.

Table on the following page summarizes comparison scenarios between SSDM and other methods. It is clear that SSDM represents an advantage with respect to other SE methods in carriers with low dimensionality as seen in Tables 7.2c and 7.2b. However, Table 7.2a displays that the BER of SSDM quickly increases along with the dimension of the carriers. 


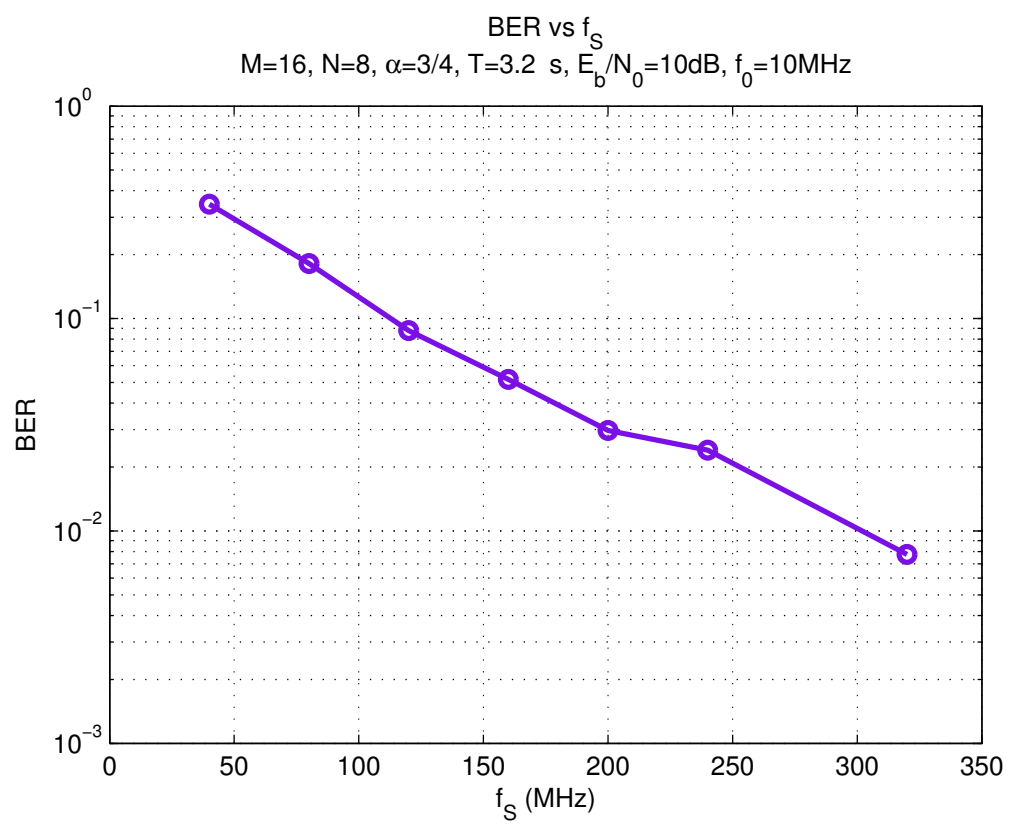

Figure 7.6: SSDM error rate BER in relation to the sampling frequency $f_{S}$

\begin{tabular}{|c|c|c|}
\hline Detector & BER & ESE $(\mathrm{bps} / \mathrm{Hz})$ \\
\hline \hline SSDM & $5 \times 10^{-1}$ & 1.60 \\
\hline Stripe & $7 \times 10^{-3}$ & 2.18 \\
\hline FSD-TSVD & $7 \times 10^{-3}$ & 2.18 \\
\hline OFDM & - & 1.90 \\
\hline
\end{tabular}
(a) $N=32, M=4$, $E_{b} / N_{0}=5 \mathrm{~dB}, \alpha=7 / 8$

\begin{tabular}{|c|c|c|}
\hline Detector & BER & ESE $(\mathrm{bps} / \mathrm{Hz})$ \\
\hline \hline SSDM & $<10^{-5}$ & 2.22 \\
\hline Precoded & $6 \times 10^{-2}$ & 2.21 \\
\hline FSD-TSVD & $10^{-1}$ & 2.00 \\
\hline OFDM & - & 1.9 \\
\hline
\end{tabular}

(b) $N=12, M=4$, $E_{b} / N_{0}=0 \mathrm{~dB}, \alpha=4 / 5$

Table 7.2: Final comparison

\begin{tabular}{|c|c|c|}
\hline Detector & BER & ESE $(\mathrm{bps} / \mathrm{Hz})$ \\
\hline \hline SSDM & $<10^{-5}$ & 2.21 \\
\hline Stripe & $10^{-1}$ & 1.99 \\
\hline ML & $8 \times 10^{-2}$ & 2.03 \\
\hline OFDM & - & 1.78 \\
\hline
\end{tabular}

(c) $N=8, M=4$, $E_{b} / N_{0}=1 \mathrm{~dB}, \alpha=3 / 4$ 


\subsection{Future work}

While the SSDM system is a novel approach to generate and decode SE signals, it is also an initial work. There is a great amount of opportunities to continue developing this methodology. In this section, the author considers elements of investigation related to SSDM.

- MIMO systems multiply the capacity, efficiency and robustness of the channel. In fact, adaptations for MIMO systems have been already done for complex algorithms such as SD [41, 43]. Therefore, the simplicity and great performance of the SSDM detector could of great value in MIMO systems.

- SSDM can also be analyzed with carriers using any type of pulse shapes. One example of this is the reception of signals that have been distorted by channels with a known frequency signature. Where the SSDM transmitter uses a uniform pulse shape for all the subcarriers, the receiver can adapt the normalized pulse shapes to adjust to the conditions of the channel without the need of equalizations.

- When two or more OFDM carriers are lined up side by side in the radioelectric spectrum, each one of them creates interference with the other. This interference is considered noise from the perspective of the receivers. If the receivers were capable of listening not only to the actual carrier but to the carriers in the sides with SSDM, the interference will rather become additional information. This feature could be called surrounding carrier awareness. Early experiments during the development of this work show that this idea is feasible. It consists on decoding several carriers at once so the samples from the interfering carrier help the detector performance.

- The approach of the SSDM receiver in this thesis requires high sampling frequencies of the incoming signal. It is relevant to analyze the SSDM radio in baseband in order to relax this constrain.

- The incoming signals to the radio can be windowed so that the spectrum is modified. For instance a Hanning window of same length of the symbol, as seen in Section 4.2 .2 on page 48 , widens the spectrum in about $4 / T$ in each side. Preliminary results shown that filtering increases both BER and the need of SNR. However, it is 

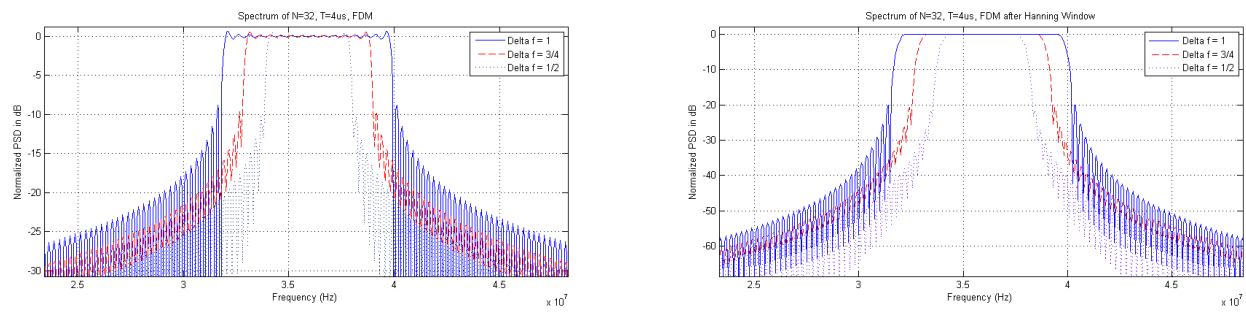

Figure 7.7: Slope change in regular and a widowed SSDM signal

also seen that the side lobes of surrounding carriers are attenuated with a pronounced slope as shown in Figure 7.7. Therefore, windowing can be tested as an alternative to reduce the interference from other carriers. By doing this, the SNR level of the signal is increased due to the reduction of the noise floor due to external interference. Moreover, it is seen in Figure 7.9 that $56 \%$ of the spectral energy of a regular RF pulse is outside the main lobe. This is a potential problem for SE systems with frequency selective channels because the side lobes of the subcarrier sync functions can be miscalculated. The spectrum of a Hanning windowed pulse, in contrast, contains $97 \%$ of the energy within the main lobe - despite of the spectrum widening. The additional increase of PAPR due to windowing as seen in Figure 7.8 is not a concern because it can happen digitally at the receiver and only for signal processing purposes. In this case, Figure 7.8 shows the application of the Hanning window on the symbol in Fig. 3.3 on page 20.

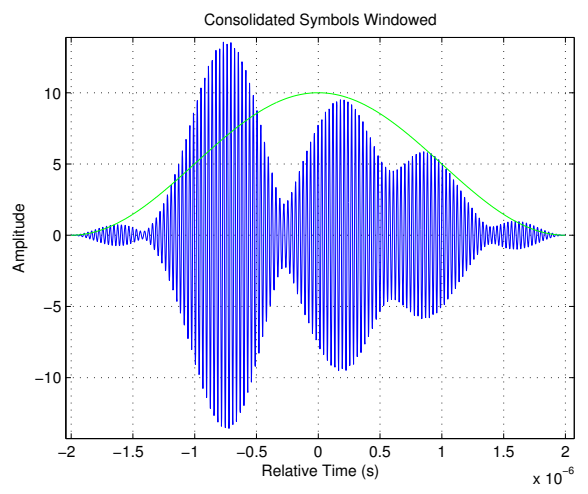

Figure 7.8: Application of a Hanning window on a symbol 


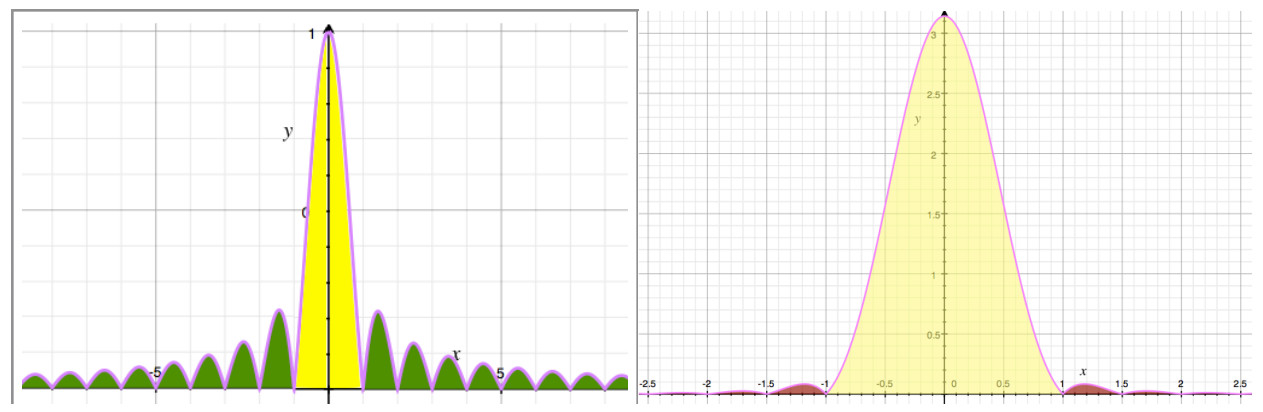

Figure 7.9: Concentration of the spectral energy before and after windowing

- The structure of the projection matrix $\Gamma$ can be studied to determine several factors. One of them is to measure the ideality of the pulse shapes for the orthonormal basis of the SSDM system. This will help make a better selection of signals shapes.

- The SSDM detector as implemented in this thesis work, requires only $N$ samples form the FFT block. It would be interesting to study a mechanism that takes more samples to perform a more complex symbol approximation. For instance, there could be a voting mechanism that assess the likelihood of accuracy of the received symbols based on a indicator or simply by selecting two out of three.

- Congested zones in cities where the spectrum is scarce are also zones of high levels of signal. That provides an inherent SNR budget that can be used to to increase the subcarrier density with SSDM. It would be meaningful to study if the implementation of SSDM in communication networks would automatically increase the capacity of such networks with no need of additional spectrum licenses or infrastructure to recycle spectrum.

- It is important to analyze the benefits of SSDM towards a potential market. SSDM could be used by equipment manufacturers to produce radios with higher SE. Similarly, standardizing organizations such as the International Telecommunication Union (ITU), the Global System for Mobile communications Association (GSMA), the European Telecommunications Standards Institute (ETSI), the Alliance for 
Telecommunications Industry Solutions (ATIS) or the Institute of Electrical and Electronic Engineers (IEEE) could use SSDM to develop the standards for future communications. In each of these cases, an assessment of the benefits and viability of deploying of SSDM needs to be executed.

\subsection{Conclusions}

The cost to achieve reduced spectrum utilization, while keeping a target BER in the communications link, is higher SNR than OFDM. As the frequency step is reduced, the detector reaches an asymptotic BER that does not improve when the SNR increments. Along with this, a more powerful architecture is required in comparison with OFDM. The receiver requires the addition of a detection block, higher sampling frequencies and longer Fourier transform blocks. Despite of this, it is seen that the SSDM transceiver is still in many ways less complex than other SE methods.

It has been proven that SSDM displays higher throughput than OFDM. Under certain conditions, SSDM also has a decreased BER and higher ESE than other SE methods. For low dimensionality carriers, the increase in throughput can be $60 \%$ more than OFDM and $8 \%$ more than other SE methods with a thousand times less error likelihood. High dimensional carriers however require the leverage on other techniques for an efficient reception.

A great benefit of SSDM is the ability to modulate and demodulate non uniform carriers and arbitrary frequency steps. This provides greater maneuverability of the carriers. Additionally, SSDM has the inherent ability to decode several carriers at once and to decode signals after windowing with practically no increment in the complexity of the receiver. 


\section{Appendix A}

\section{Fractional Discrete Fourier Transform (FrDFT)}

The general formula of the direct form of the Discrete Fourier Transform (DFT) is given by

$$
X_{k}=\sum_{n=0}^{N-1} x_{n} \cdot e^{-j 2 \pi \frac{k}{N} n} .
$$

Where a regular DFT block calculates all the possible $X_{k}$, the FrDFT does only $m$ of the $X_{k}$. The selected $X_{k}$ correspond to the $m$ spectral samples of interest. $k$ is then restricted to $\left\{k_{1}, \ldots, k_{m}\right\}$. Adapted to SE receivers, these expressions become $\mathbf{S}\left[f_{i}\right]=\sum_{k^{\prime}=-T f_{S} / 2}^{T f_{S} / 2-1} \mathbf{S}\left[t_{k^{\prime}}\right] \cdot e^{-j 2 \pi f_{i}}$ where $t_{k^{\prime}}=k^{\prime} T_{s}$ and $f_{i}$ every element in $\left\{-f_{S} / 2,-f_{S} / 2+1 / T, \ldots, f_{S} / 2-1 / T\right\}$. Where a regular DFT block calculates $\mathbf{S}\left[f_{i}\right]$ for every possible $f_{i}$, the FrDFT does only for $N$ of the $f_{i}$. The $f_{i}$ selected correspond to the spectral samples of interest. This is, $\left\{\mathbf{S}\left[f_{a}\right], \mathbf{S}\left[f_{b}\right], \ldots, \mathbf{S}\left[f_{z}\right]\right\}$.

\section{Inverse Fractional Discrete Fourier Transform (IFrDFT)}

Similarly, the general formula of the direct form of the Inverse Discrete Fourier Transform (IDFT) is given by

$$
x_{n}=\frac{1}{N} \sum_{k=0}^{N-1} X_{k} e^{i 2 \pi k / N n} .
$$

A regular IDFT block calculates each $x_{n}$ using all the possible $k$. However, the IFrDFT block calculates each $x_{n}$ using only $m$ elements from $k$. The selected $k$ correspond to the modulating inputs (no padding) of the block. Where the regular DFT calculates every possible $k_{i}$, the FrDFT does only for $N$ of the $k_{i}$. This is, $\left\{k_{a}, k_{b}, \ldots, k_{z}\right\}$. This 
replaces 7.1 for $x_{n}=\frac{1}{N}\left(X_{k_{1}} e^{i 2 \pi^{k} / N n}+\ldots+X_{k_{m}} e^{i 2 \pi k_{m} / N n}\right)$. Apart from this, for an output $x_{n_{1}, n_{2}, \ldots, n_{d}}$ consisting of real data, the input vector shall have a conjugate symmetry $X_{k_{1}, k_{2}, \ldots, k_{m}}=X_{N-k_{1}, N-k_{2}, \ldots, N-k_{m}}^{*}$. Therefore, an output element of the IFrDFT is

$$
x_{n}=\frac{1}{N}\left(X_{k_{1}} e^{i 2 \pi k_{1} / N n}+X_{k_{1}}^{*} e^{i 2 \pi N-k_{1} / N n}+\ldots+X_{k_{m}} e^{i 2 \pi k_{m} / N n}+X_{k_{m}}^{*} e^{i 2 \pi N-k_{m} / N n}\right)
$$

Adapted to SE receivers, these expressions become $\mathbf{s}[t]=\frac{1}{T f_{S}} \sum_{k^{\prime}=-T f_{S} / 2}^{T f_{S} / 2-1} \mathbf{S}\left[f_{k^{\prime}}\right] \cdot e^{j 2 \pi f_{k^{\prime}}}$ from which not all the $k^{\prime}$ are used but only the ones associated to the frequencies of interest $\left\{f_{a}, f_{b}, \ldots, f_{z}\right\}$. Therefore, this expression becomes

$$
\mathbf{S}[t]=\frac{1}{T f_{S}}\left(\mathbf{S}\left[f_{a}\right] e^{j 2 \pi f_{a}}+\mathbf{S}^{*}\left[f_{a}\right] e^{j 2 \pi f_{a}}+\ldots+\mathbf{S}\left[f_{m}\right] e^{j 2 \pi f_{m}}+\mathbf{S}^{*}\left[f_{m}\right] e^{j 2 \pi f_{m}}\right) .
$$




\section{Appendix B}

\section{The Gram Schmidt (GS) procedure [5]}

In mathematics the Gram Schmidt orthonormalization procedure is a method of orthonormalisation used to find an orthogonal normalized base from a set of either vectors or functions.

Given a finite or countably-finite linearly-independent set $\left\{x_{i}\right\}_{i=1}^{N}\left(x_{i}^{\prime}\right.$ s distinct, $\left.1 \leq N \leq \infty\right)$, the Gram Schmidt orthonormalization procedure is a recursive process by which to convert it into an orthonormal set t $\left\{y_{i}\right\}_{i=1}^{N}$ for which $\operatorname{span}\left\{y_{i}\right\}_{i=1}^{M}=\operatorname{span}\left\{x_{i}\right\}_{i=1}^{N}$, with $M=N$.

First, let $y_{1}=\frac{x_{1}}{\left\|x_{1}\right\|}$. If $N=1$, then stop; otherwise, for $i=2, \ldots, N$, let $z_{i}=$ $\sum_{k=1}^{i-1}\left[x_{i}, y_{i}\right] y_{k}$, and then let $y_{i}=\frac{x_{i}-z_{i}}{\left\|x_{i}-z_{i}\right\|} \cdot[$,$] denotes the inner product operation.$

Example:

$$
\begin{array}{rlrl}
\mathbf{u}_{1} & =\mathbf{x}_{1}, & \mathbf{y}_{1} & =\frac{\mathbf{u}_{1}}{\left\|\mathbf{u}_{1}\right\|} \\
\mathbf{u}_{2} & =\mathbf{x}_{2}-\left[\mathbf{x}_{2}, \mathbf{y}_{1}\right] \mathbf{y}_{1} & \mathbf{y}_{2} & =\frac{\mathbf{u}_{2}}{\left\|\mathbf{u}_{2}\right\|} \\
\mathbf{u}_{3} & =\mathbf{x}_{3}-\left[\mathbf{x}_{3}, \mathbf{y}_{1}\right] \mathbf{y}_{1}-\left[\mathbf{x}_{3}, \mathbf{y}_{2}\right] \mathbf{y}_{2}, & \mathbf{y}_{3} & =\frac{\mathbf{u}_{3}}{\left\|\mathbf{u}_{3}\right\|} \\
& \vdots & \vdots \\
\mathbf{u}_{k} & =\mathbf{x}_{k}-\sum_{k=1}^{i-1}\left[\mathbf{x}_{i}, \mathbf{y}_{i}\right] \mathbf{y}_{k}, & \mathbf{y}_{k} & =\frac{\mathbf{u}_{k}}{\left\|\mathbf{u}_{k}\right\|}
\end{array}
$$




\section{Bibliography}

[1] Supplement to ieee standard for information technology - telecommunications and information exchange between systems - local and metropolitan area networks - specific requirements. part 11: Wireless lan medium access control (mac) and physical layer (phy) specifications: High-speed physical layer in the $5 \mathrm{ghz}$ band. IEEE Std 802.11a-1999, page i, 1999.

[2] S. I. A. Ahmed. Spectrally Efficient FDM Communication Signals and Transceivers: Design, Mathematical Modelling and System Optimization. PhD thesis, University College London, Communications and Information Systems Research Group, 10 2011.

[3] S. Bharadwaj, B. Nithin Krishna, V. Sutharshun, P. Sudheesh, and M. Jayakumar. Low complexity detection scheme for nofdm systems based on ml detection over hyperspheres. In Devices and Communications (ICDeCom), 2011 International Conference on, pages 1-5, feb. 2011.

[4] Blogial. (2012, May 19). LTE - Long Term Evolution. [Online]. Available: http: //blogial.com/2009/09/20/lte-long-term-evolution/.

[5] J. R. Breitenbach. Digital communications - supplementary notes. El Corral Bookstore, 1 Grand Ave, San Luis Obispo, CA 93401, 2011.

[6] S. C. Byeong Lee. Broadband Wireless Access and Local Networks: Mobile WiMAX and WiFi. Artech House, 2008.

[7] S. C. Carroll. Non-orthogonal frequency-division multiplexed communication through a non-linear transmission medium. Patent, 12 2010. US 7852745. 
[8] R. Clegg, S. Isam, I. Kanaras, and I. Darwazeh. A practical system for improved efficiency in frequency division multiplexed wireless networks. Communications, IET, 6(4):449 -457, 62012.

[9] CNN Money. (2012, May 16). LightRadio: The tiny cube that could save wireless. [Online]. Available: http://www.ctia.org/advocacy/research/index. cfm/aid/10323.

[10] CNN Money. (2012, May 16). Spectrum crunch is creating wireless carrier mergers. [Online]. Available: http://money.cnn.com/2012/02/21/technology/ spectrum_crunch/index.htm.

[11] CNN Money. (2012, May 16). Spectrum Crunch: The cell phone industry hits its limits. [Online]. Available: http://money.cnn.com/2012/02/21/technology/ spectrum_crunch/index.htm.

[12] CNN Money. (2012, May 16). U. S. Wireless Quick Facts. [Online]. Available: http://money.cnn.com/2012/02/24/technology/spectrum_ crunch_solutions/index.htm.

[13] C. Cox. An Introduction to Lte: Lte, Lte-Advanced, Sae and $4 g$ Mobile Communications. John Wiley \& Sons, Ltd., 2012.

[14] CTIA-The Wireless Association. (2012, May 16). U. S. Wireless Quick Facts. [Online]. Available: http://www.ctia.org/advocacy/research/index.cfm/aid/ 10323.

[15] M. Engels. Wireless Ofdm Systems: How to Make Them Work? The Kluwer International Series in Engineering and Computer Science. Kluwer Academic Publishers, 2002.

[16] Federal Communications Commission (FCC). (2012, May 16). FCC Auctions: All Auctions:: [Online]. Available: http://wireless.fcc.gov/auctions/default. htm?job=auctions_all. 
[17] F. F. Fulton. Receiver for receiving non orthogonal multiplexed signals. Patent, 05 1966. US 3456195.

[18] R. Grammenos, S. Isam, and I. Darwazeh. Fpga design of a truncated svd based receiver for the detection of sefdm signals. In Personal Indoor and Mobile Radio Communications (PIMRC), 2011 IEEE 22nd International Symposium on, pages 2085 -2090 , sept. 2011.

[19] S. Isam and I. Darwazeh. Precoded spectrally efficient fdm system. In Personal Indoor and Mobile Radio Communications (PIMRC), 2010 IEEE 21st International Symposium on, pages $99-104$, sept. 2010.

[20] S. Isam and I. Darwazeh. Design and performance assessment of fixed complexity spectrally efficient fdm receivers. In Vehicular Technology Conference (VTC Spring), 2011 IEEE 73rd, pages 1-5, may 2011.

[21] S. Isam, I. Kanaras, and I. Darwazeh. A truncated svd approach for fixed complexity spectrally efficient fdm receivers. In Wireless Communications and Networking Conference (WCNC), 2011 IEEE, pages 1584 -1589, march 2011.

[22] W. Jian, Y. Xun, Z. Xi-lin, and D. Li. The prefix design and performance analysis of dft-based overlapped frequency division multiplexing (ovfdm-dft) system. In Signal Design and Its Applications in Communications, 2007. IWSDA 2007. 3rd International Workshop on, pages 361 -364, sept. 2007.

[23] I. Kanaras. Spectrally Efficient Multicarrier Communication Systems: Signal Detection, Mathematical Modeling and Optimization. PhD thesis, University College London, Communications and Information Systems Research Group, 062012.

[24] I. Kanaras, A. Chorti, M. Rodrigues, and I. Darwazeh. Investigation of a semidefinite programming detection for a spectrally efficient fdm system. In Personal, Indoor and Mobile Radio Communications, 2009 IEEE 20th International Symposium on, pages $2827-2832$, sept. 2009. 
BIBLIOGRAPHY

[25] I. Kanaras, A. Chorti, M. Rodrigues, and I. Darwazeh. Spectrally efficient fdm signals: Bandwidth gain at the expense of receiver complexity. In Communications, 2009. ICC '09. IEEE International Conference on, pages 1-6, jun 2009.

[26] I. Kanaras, A. Chorti, M. Rodrigues, and I. Darwazeh. A fast constrained sphere decoder for ill conditioned communication systems. Communications Letters, IEEE, 14(11):999 -1001, november 2010.

[27] A. Kliks, H. Bogucka, I. Stupia, and V. Lottici. A pragmatic bit and power allocation algorithm for nofdm signalling. In Wireless Communications and Networking Conference, 2009. WCNC 2009. IEEE, pages 1-6, april 2009.

[28] D. LI. Method and system of frequency division multiplexing. Patent Application, 03 2011. US 2011/0075649 A1.

[29] H. Liu and G. Li. OFDM-Based Broadband Wireless Networks: Design And Optimization. Wiley-Interscience, 2005.

[30] F. H. G. Matz. Wireless communications over rapidly time-varying channels. Oxford, UK; Burlington, MA: Academic Press,, 2011.

[31] F. ming Han and X. da Zhang. Wireless multicarrier digital transmission via weylheisenberg frames over time-frequency dispersive channels. Communications, IEEE Transactions on, 57(6):1721 -1733, june 2009.

[32] A. Osseiran, J. Monserrat, and W. Mohr. Mobile and Wireless Communications for IMT-Advanced and Beyond. John Wiley \& Sons, Ltd., 2011.

[33] S. Pagadarai, A. Kliks, H. Bogucka, and A. Wyglinski. On non-contiguous multicarrier waveforms for spectrally opportunistic cognitive radio systems. In Waveform Diversity and Design Conference (WDD), 2010 International, pages 000177 -000181, aug. 2010.

[34] M. Parker. Digital Signal Processing: Everything You Need to Know to Get Started. Elsevier Science, 2010. 
[35] H. Rohling. OFDM Concepts for Future Communication Systems. Springer Berlin Heidelberg, 2011.

[36] S. Smith. Digital Signal Processing: A Practical Guide for Engineers and Scientists. Demystifying Technology Series. Newnes, 2003.

[37] Y. Sun, H. Wang, and Y. Zhang. Fast detection algorithm based on sphere decoding for overlapped hybrid division multiplexing system. In Future Computer and Соттиnication (ICFCC), 2010 2nd International Conference on, volume 1, pages V1-277 -V1-280, may 2010.

[38] A.-E. M. Taha, H. S. Hassanein, and N. A. Ali. Enabling Technologies for IMTAdvanced Networks, pages 19-37. John Wiley \& Sons, Ltd., 2011.

[39] H. Taoka and K. Higuchi. Experiments on peak spectral efficiency of $50 \mathrm{bps} / \mathrm{hz}$ with 12-by-12 mimo multiplexing for future broadband packet radio access. In Communications, Control and Signal Processing (ISCCSP), 2010 4th International Symposium on, pages $1-6$, march 2010.

[40] Texas Instruments. (2012, May 16). Direct RF Sampling ADCs - Breakthrough RFSampling ADCs Revolutionize Radio Architectures. [Online]. Available: http:// www.ti.com/ww/en/analog/dataconverters/rf_sampling_adc.shtml.

[41] Y. Zhang, J. Tang, and K. Parhi. Low complexity radius reduction method for list sphere decoders. In Signals, Systems and Computers, 2006. ACSSC '06. Fortieth Asilomar Conference on, pages 2200 -2203, 29 2006-nov. 12006.

[42] J. Zhao. Analysis and Design of Communication Techniques in Spectrally Efficient Wireless Relaying Systems. PhD thesis, Swiss Federal Institute of Technology (ETH), Zurich, jul 2010.

[43] C. Zheng, X. Chu, J. McAllister, and R. Woods. Real-valued fixed-complexity sphere decoder for high dimensional qam-mimo systems. Signal Processing, IEEE Transactions on, 59(9):4493 -4499, sept. 2011. 